\title{
Preliminary Angiosperm Checklist in an Area South of the Madeira River, Manicoré, Amazonas, Brazil
}

\author{
Caroliny Almeida Coelho ${ }^{a^{*}}$, Marta Regina da Silva Pereirab, Bruno Sampaio Amorim,d \\ a Universidade do Estado do Amazonas, Manicoré, 69280-000, Amazonas, Brazil.* caarolinyalmeida07@gmail.com \\ b Instituto Nacional de Pesquisas da Amazônia, Manaus, 69067-375, Amazonas, Brazil. \\ c Programa de Pós-Graduação em Biotecnologia e Recursos Naturais, Universidade do Estado do Amazonas, Manaus, 69058-807, \\ Amazonas, Brazil. \\ d Museu da Amazônia, Manaus, 69099-415, Amazonas, Brazil.
}

Received: September 14, 2019 / Accepted: November 21, 2019 / Published online: January 20, 2020

\begin{abstract}
Due to the large extent of the Amazon rainforest, research has historically focused on easily accessible locations. Thus, much of this region has little information available about its richness and plant distribution. Located south of the Madeira river, Municipality of Manicoré has a high number of phytophysiognomies, which may indicate the existence of a greater diversity of plant species. Therefore, from the compilation of previously collected records and based on botanical expeditions, this study evaluated the diversity and richness of angiosperms in Manicoré. We found 801 species, 409 genera and 106 families. Our data record 47 new occurrences for Amazonas State. Of these new occurrences, 12 are also the first record for the northern region. In addition, we have identified a new vine species of the genus Mandevilla Lindl. Given the well-known sample deficiency of the Amazon region, and considering the countless anthropogenic pressures that cities south of the Madeira river have been facing, knowledge of flora becomes increasingly urgent.
\end{abstract}

Keywords: Brazilian Flora, Amazon Rainforest, Collection Gaps, Taxonomic novelties, Anthropogenic Pressures.

\section{Checklist preliminar de angiospermas em uma área ao sul do rio Madeira, Manicoré, Amazonas, Brasil}

\section{Resumo}

Devido a grande extensão da Floresta Amazônica, historicamente as pesquisas tem se concentrado em locais de fácil acesso. Sendo assim, grande parte desta região apresenta poucas informações disponíveis sobre sua riqueza e distribuição de plantas. Localizado ao sul do rio Madeira, o município de Manicoré apresenta um elevado número de fitofisionomias, o que pode indicar a existência de uma maior diversidade de espécies vegetais. Diante disso, o objetivo deste trabalho é avaliar a diversidade e riqueza de angiospermas do município de Manicoré, a partir da compilação de registros coletados anteriormente e da realização de expedições botânicas. Foram listadas 801 espécies, 409 gêneros e 106 famílias. Nossos dados registram 47 novas ocorrências para o Estado do Amazonas, sendo que destas novas ocorrências, 12 são também o primeiro registro para a Região Norte. Além disso, identificamos uma nova espécie de trepadeira do gênero Mandevilla Lindl. Diante da conhecida deficiência amostral da região Amazônica, e considerando as inúmeras pressões antrópicas que os municípios da região sul do rio Madeira vem enfrentando, o conhecimento da flora se torna cada vez mais urgente.

Palavras-chave: Flora brasileira, Floresta Amazônica, Lacunas de coletas, Novidades taxonômicas, Pressões antrópicas.

\section{Introduction}

The Amazon rainforest is an extensive cluster of landscapes and ecosystems that form an exuberant mosaic of vegetation and hydrography. Its area covers 8 million $\mathrm{km}^{2}$, distributed in nine South American countries (Araújo, 2008; PortoGonçalves, 2015). It is the largest tropical rainforest in the world, being considered the largest reservoir of biodiversity on the planet (Porto-Gonçalves, 2015), besides being the main source of all biodiversity in the neotropical region (Antonelli et al., 2018).
Despite containing all this biodiversity, much of the Amazon rainforest is still unknown and for many reasons the most of its area remain undersampled (Hopkins, 2007; ter Steege et al., 2016). Approximately 33,300 angiosperm species are listed for Brazil, of which more than 12,000 occur in the Amazon and more than 8,500 in Amazonas State (Flora do Brasil 2020, under construction). However, estimates of the total number of species are much higher (BFG, 2015; ter Steege et al., 2016; Domingos et al., 2017). Due to the large extent of the Amazon rainforest, research 
has focused on easily accessible locations such as forest areas near the urban centers of large cities (Hopkins, 2007; BFG, 2015). Thus, much of the Amazon basin, in hard-to-reach areas, has little available information about its richness and plant distribution (Hopkins, 2007, 2019; Forzza et al., 2010).

The Madeira river, which makes up the Amazon basin, has a total length of approximately $3,240 \mathrm{~km}$, of which about 1,425 $\mathrm{km}$ are located in Brazil (Siqueira, 2013). Located on the south side of the river, the municipality of Manicore has a high number of plant typologies, which may indicate a great diversity of plant species (Silva \& Pereira, 2005). Nevertheless, Manicoré is inserted in an area of little botanical research, with a high proportion of unidentified species, and is likely to contain many species not yet described (Hopkins, 2007; Forzza et al., 2010; BFG, 2015; ter Steege et al., 2016).

Considering anthropogenic actions aimed at economic interests, such as illegal logging and timber processing (Fearnside, 2006), land grabs (Silva \& Pereira, 2005; Carvalho, 2010), and the existence of countless gold rafts that cause siltation of rivers and can destroy vegetation even before it is known (Siqueira, 2013), it is essential to draw up a floristic list for the municipality.

Therefore, this study evaluated the diversity and richness of angiosperms in Manicoré and recorded new species for the Amazon.

\section{Materials and Methods}

\section{Study area characterization}

The municipality of Manicoré $\left(05^{\circ} 48^{\prime} 33^{\prime}\right.$ ' $\mathrm{S}, 61^{\circ} 18^{\prime} 01^{\prime}$, W) covers an area of approximately $48,315 \mathrm{~km}^{2}$ and is located in northern Brazil, south of Amazonas State, on the banks of the Madeira river (IBGE, 2017; Figure 1).

According to the Köppen classification, the climate of the region is type Am, monsoon, with annual rainfall ranging from
$2,250 \mathrm{~mm}$ to $2,750 \mathrm{~mm}$. The highest rainfall rates occur from January to March, with a short dry season in July (Brasil, 1978). The average annual temperature ranges from $24^{\circ} \mathrm{C}$ to $26^{\circ} \mathrm{C}$, and the relative humidity is very high, ranging from 85 to $90 \%$ (Brasil, 1978). The topography is flat, with welldrained soil, predominantly classified as Alic Yellow Latosol - A, originated from sediments of the Solimões Formation. The litter layer reaches approximately $10 \mathrm{~cm}$ in height (Brasil, 1978).

The vegetation cover of the area is predominantly classified as Lowland Dense Ombrophilous Forest in association with other plant typologies, accounting for approximately $65.4 \%$ of the existing natural vegetation (Silva \& Pereira, 2005; Figure 2). Other registered vegetation types are Submontane Dense Ombrophilous Forests and, along the main rivers, Open Alluvial Ombrophilous Forests and Dense Alluvial Ombrophilous Forests occur to a lesser extent (IBGE, 2004).

\section{Data collection}

Records of angiosperm collections in Manicoré were obtained from the database of the Herbarium Collection of the National Institute for Amazonian Research - INPA and from the digital collection of the Environmental Information Reference Center - CRIA (2018), via consultation in the collections of the following herbaria: ASU, BHCB, CEN, CGMS, EAFM, HRCB, HUEFS, HUFSJ, NYBG, MBM, MO, RON, SP, SPF, UB, UEC, UFG, UNOP, UPCB, and US, from May to August 2018 (herbarium acronyms according to Thiers, continuously updated).

With the purpose of collecting new samples for the region, 28 botanical expeditions were carried out from May 2018 to February 2019 through random walks in forest areas of different phytophysiognomies. These expeditions prioritized 'terra firme' forests, meadows, and campinaranas, totaling approximately $90 \mathrm{~h}$ of field sampling in these areas.

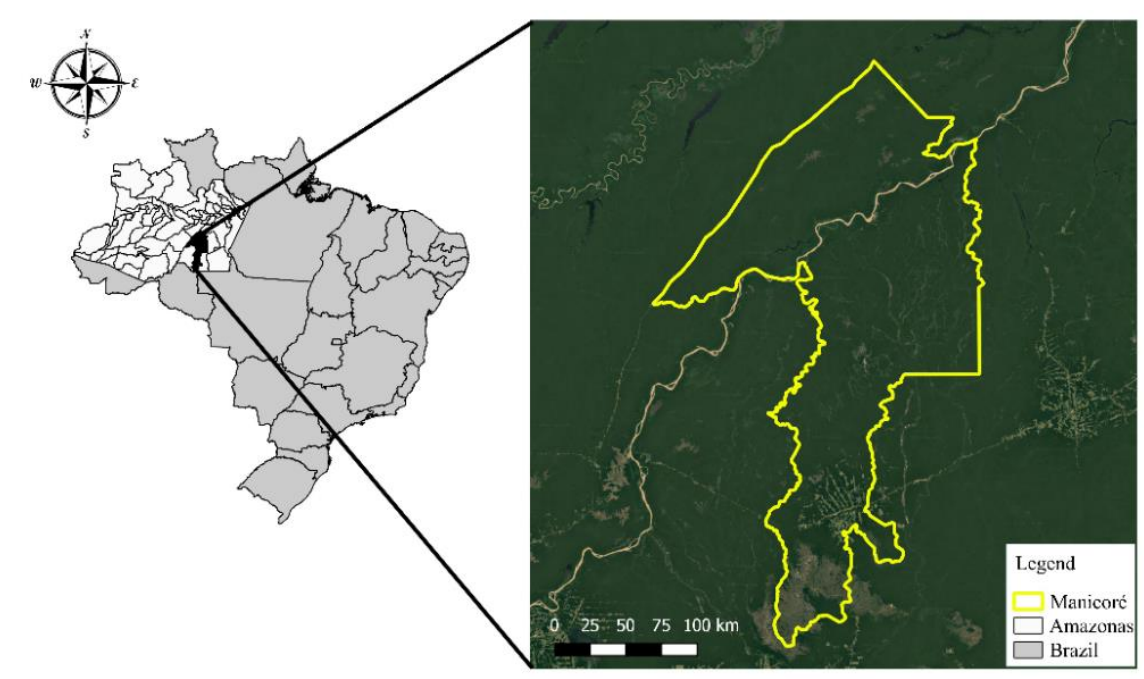

Figure 1. Municipality of Manicoré, Amazonas State, Brazil.

The botanical material was collected according to guidelines for herbarium collection (Peixoto \& Maia, 2013).

To classify species habit, the definitions presented by Gonçalves and Lorenzi (2007) were considered. For the samples obtained from the herbarium database, the habits described by the collectors were considered.

To identify the species, we used specialized bibliographies and comparisons between images available in 
the digital herbarium of the Botanical Garden of Rio de Janeiro - JABOT (2018), CRIA (2018), and Flora do Brasil 2020 (under construction), in addition to consultations with experts. After identification, the material was incorporated into the
INPA herbarium, with duplicates in the HUEFS, RB, and UPF herbariums (herbarium acronyms according to Thiers, continuously updated).

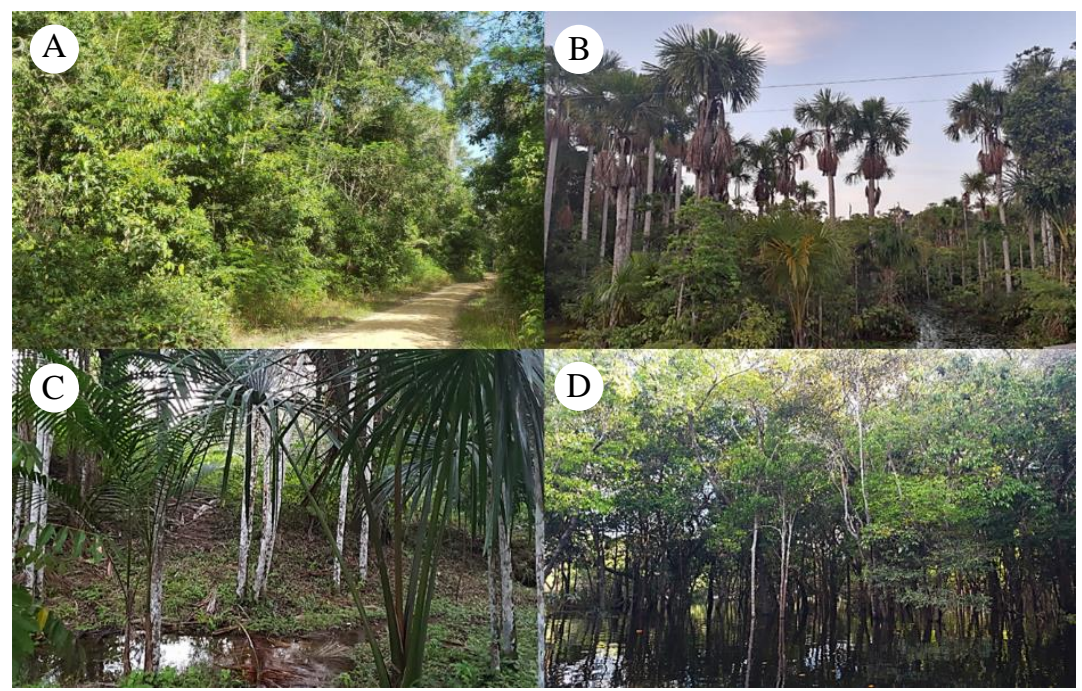

Figure 2. Phytophysiognomies. A. Lowland Dense Ombrophilous Forest. B. Lowland Dense Ombrophilous Forest associated with Palm Trees. C. Open Alluvial Ombrophilous Forest. D. Dense Alluvial Ombrophilous Forest. (Photos by Caroliny Almeida Coelho).

Flora do Brasil 2020 (under construction) was consulted to verify species nomenclature and confirm the records for Amazonas State and by phytogeographic domain. When this was unavailable, the synonyms and spellings of the taxa were updated by consulting The Plant List (2010) database.

Specimens unidentified at the species level were not included in the checklist, which contains only one voucher per species occurring in Manicoré.

The map of the location of the studied municipality (Figure 1) was generated in ArcGIS 10.3. The map of the distribution of angiosperm collections in the municipality was generated in Quantum GIS 1.7. For the elaboration of this map, geographic coordinates available in the INPA and CRIA databases (2018) were used. However, 313 species were not included because records did not have geographic coordinates or because species were incorrectly georeferenced. The map of angiosperm records for cities south of the Madeira river was also generated in Quantum GIS 1.7, using data obtained from the CRIA digital archive (2018), which were manually analyzed and filtered.

\section{Results and Discussion}

A total of 106 families, 409 genera, and 801 species were listed for the municipality of Manicore (Table 1). The richest family was Fabaceae (117 spp.), followed by Euphorbiaceae (35 spp.), Rubiaceae (34 spp.), Apocynaceae (30 spp.), Melastomataceae (29 spp.), Moraceae (27 spp.), Arecaceae (24 spp.), Myrtaceae (20 spp.), Malvaceae (19 spp.), Annonaceae, and Burseraceae (18 spp. each), accounting for $46.3 \%$ of the listed species.

For Brazil (2,756 spp.), as well as for the Amazon biome (1,119 spp.) and Amazonas State (825 spp), Fabaceae is the family with the highest species richness (BFG, 2015; Fabaceae in Flora do Brasil 2020, under construction). In the Ducke Reserve Flora Project, which is the best studied area of the Brazilian Amazon, Fabaceae (still considered in the circumscription of subfamilies Mimosoideae - 68 spp., Papilionoideae - 66 spp., and Caesalpinioideae - 54 spp.) is also the best represented botanical family (Ribeiro, Nelson, Silva, Martins \& Hopkins, 1994; Hopkins, 2005). In addition to this family, all the other richest families in our study (except Malvaceae) are in the ranking of the thirty richest families in number of taxa, being also found in the Ducke Reserve (Ribeiro et al., 1994; Hopkins, 2005).

Other studies also conducted in the Amazon showed that Fabaceae has been consistently cited as one of the families with the largest number of species (Amaral, Matos \& Lima, 2000; Oliveira et al., 2008; Silva, Matos \& Ferreira, 2008; Pinheiro et al., 2010). The presence of representatives of Fabaceae, Euphorbiaceae, Moraceae, and Malvaceae among the richest families is a common point among the floristic surveys conducted in the Amazon Forest (Gonçalves \& Santos, 2008; Sardinha, Freitas, Santos, Cruz-Junior \& Santos, 2017).

Of the 409 genera, the most representative are Inga and Miconia (16 spp. each), Protium (13 spp.), Byrsonima, Myrcia, Palicourea, and Swartzia (10 spp. each), Bactris and Eugenia (8 spp. each), Annona, Piper, and Senna (7 spp. each), accounting for $29.8 \%$ of the angiosperm richness of Manicoré. This composition is similar to that pointed out by Ribeiro et al. (1994), who cite Miconia (27 spp.), Protium (24 spp.), Inga (21 spp.), and Swartzia (19 ssp.) among the ten most representative genera in the Ducke Reserve Flora Project. Oliveira et al. (2008) and Pinheiro et al. (2010) also cite Protium and Swartzia among the most representative genera in other studies conducted in the Amazon. 
Also corroborating our findings, a study of a forest fragment in southwestern Amazonia mentions Inga, Miconia, and Byrsonima as the richest genera (Oliveira, Nagy, Barros, Martins \& Murta-Junior, 2015).

Of the remaining genera, 256 are represented by only one species, 68 by two species, and 30 by three species, which together amount to $44.2 \%$ of the municipality richness. The large number of genera represented by few species may reflect the high number of plant typologies (44) found in Manicoré, as already mentioned by Silva \& Pereira (2005). Notwithstanding, other factors may also be considered, such as the absence of dispersers, nutrient-poor soils, among other factors, indicating that further studies should be carried out.

Distributed among these distinct phytophysiognomies, $45.7 \%$ (366 spp.) of the species are trees, 27.5\% (220 spp.) are shrubs, $13.2 \%$ (106 spp.) are herbs, 4.7\% (38 spp.) are lianas, $3.5 \%$ (28 spp.) are herbaceous vines, 3.2\% (26 spp.) are subshrubs, and 2.2\% (17 spp.) are palms.

In Manicoré, there is a ratio of four woody species (tree, shrub, liana, and subshrub) to one herbaceous species (herb, herbaceous vine, and palms). The predominance of trees over other types of habits has already been observed in another study conducted in the Amazon Forest (Garcia, Silva, Zonetti \& Romagnolo, 2011) and follows the general pattern recorded for the Amazon phytogeographic domain (BFG, 2015). In turn, the herbaceous habit is better represented in open areas, as already reported by Mota et al. (2018).

Regarding distribution, $49.2 \%$ (394 spp.) of the registered species are exclusive to the Amazon domain and 48.4\% (388 spp.) occur in the Amazon and other Brazilian domains, especially Cerrado (10.2\% - 82 spp.), Atlantic Forest (5\% - 40 spp.), Pantanal (0.5\% - 4 spp.), and Caatinga ( $0.4 \%$ - 3 spp.). Moreover, 29\% (232 spp.) of species share between three to five domains and 3.4\% (27 spp.) are cited for six Brazilian biomes. It is noteworthy that $1.5 \%$ (12 spp.) of the total species had no association with the Amazon phytogeographic domain so far (BFG, 2015). Therefore, Asystasia gangetica (L.) T.Anderson, Bauhinia rufa (Bong.) Steud, Byrsonima Laxiflora Griseb, Citrus $x$ aurantium L., Connarus suberosus Planch, Dioscorea hassleriana Chodat, Hibiscus sabdariffa, Libidibia ferrea (Mart. ex Tul.) L.P.Queiroz, Mandevilla sp.Nova, Ouratea spectabilis (Mart.) Engl, Paepalanthus guaraiensis Moldenke, and Persea americana Millare are considered new occurrences for the Amazon and 0.9\% (7 spp.) lack information.

Species dispersal and exchange among different phytogeographic domains are related to numerous evolutionary processes and historical geological events (Fiaschi \& Pirani, 2009; Batalha-Filho \& Miyaki, 2014). Thus, the occurrence of species with disjoint distribution patterns between the Amazon and the Atlantic Forest shows a possible connection between the floras of these regions through the Cerrado in the past (Fiaschi \& Pirani, 2009). In this sense, gallery forests were responsible for the connection between the two largest neotropical rainforests: the Amazon Rainforest and the Atlantic Forest (Méio et al., 2013).

Regarding origin, $96 \%$ (769 spp.) of the species are native and 4\% (32 spp.) exotic. During floristic-phytosociological surveys or taxonomic reviews, it is common to find exotic plants in the study areas (Moro et al., 2012). In this regard, Althernanthera tenella Colla (Amaranthaceae), Jatropha gossypifolia L. (Euphorbiaceae), Lantana camara L. (Verbenaceae), Lippia alba (Mill.) N.E.Br. ex P. Wilson (Verbenaceae), Merremia umbellata L.Hallier f. (Convolvulaceae), Mimosa invisa Mart. ex Colla (Fabaceae), and Ricinus communis L. (Euphorbiaceae) are found in disturbed areas of Manicoré. These plants are typical of secondary succession, are able to grow in adverse conditions, and are an integral part of the urbanized landscape (Souza, Machado-Filho \& Andrade, 2012). Exotic plants are more likely to be found in these areas, with only a small fraction of them being naturalized, such as L. camara and $R$. communis for example. Thus, the presence of naturalized species in the study area is a strong evidence of the anthropogenic influence on the environment (Schneider, 2007).

The degree of aggressiveness that an exotic species can present to the natural environment is not always known (Schneider, 2007). Considering that botanical surveys are the basis for establishing criteria for the prevention and control of possible damage to the natural environment, it is recommended that all naturalized or invasive exotic species be clearly named as such and recorded for the study area (Schneider, 2007; Moro et al., 2012).

In this checklist, as well as in Flora do Brasil 2020 (under construction), Asystasia gangetica (L.) T. Anderson (Acanthaceae), Cenchrus purpureus (Schumach.) Morrone (Poaceae), Citrus x aurantium L. (Rutaceae), and Gossypium barbadense L. (Malvaceae) are labeled as naturalized. In Cuba, these species are considered invasive, with high capacity for growth, proliferation, and dispersal, and are often able to compete aggressively for dominance of the environment. In this sense, C. purpureus and G. barbadense are examples of species that still behave as transformers of natural and agricultural ecosystems in Cuba (Prieto \& González-Oliva, 2015).

For floristic and phytosociological studies, it is only interesting to report the occurrence of exotic species merely cultivated to the site if they are clearly labeled in the study description (Moro et al., 2012). Thus, of the exotic species recorded here, $68.7 \%$ (22 spp.) are naturalized and 31.3\% (10 spp.) cultivated. Among exotic species, eight had not been cited for Amazonas State in Flora do Brazil 2020 (under construction) and four occur in other northern states.

Hence, Combretum indicum (L.) Jongkind [Combretaceae] (Cultivated), Cymbopogon citratus (DC.) Stapf [Poaceae], Gymnanthemum amygdalinum (Delile) Sch. Bip. ex Walp [Asteraceae] (Cultivated), and Petiveria alliacea L. [Phytolaccaceae] are recorded as new occurrences for Amazonas State. Moreover, Asystasia gangetica [Acanthaceae], Citrus x aurantium [Rutaceae], Panicum capillare L. [Poaceae], and Persea americana Mill. [Lauraceae] are recorded as new occurrences for Amazonas State and the northern region.

During field expeditions for this work, material from Hibiscus sabdariffa L. was collected from a disturbed area of secondary forest (Figure 3a-b). Coelho and Amorim (2019) found that this fact corresponds to an indication of naturalization of this species in the Brazilian Amazon. 
Prior to the study by Coelho and Amorim (2019), Hibiscus sabdariffa was only recorded as cultivated (Esteves, Duarte \& Takeuchi, 2014), being absent from the records of Brazilian angiosperms (BFG, 2015; Hibiscus in Flora do Brasil 2020, under construction).

In this checklist, as in the study by Coelho and Amorim (2019), this species is labeled as naturalized and recorded as new occurrence for Brazil (Table 1). In addition to the new occurrences of exotic species, 39 new occurrences of native species were recorded for Amazonas State, eight of which are also new occurrences for the northern region (Table 1). It is noteworthy that of the new occurrences for Amazonas State, $20.5 \%$ (8 spp.) do not occur in the Amazon phytogeographic domain. However, they used to occur exclusively in the Cerrado or associated with this domain, showing great floristic heterogeneity for the study area.

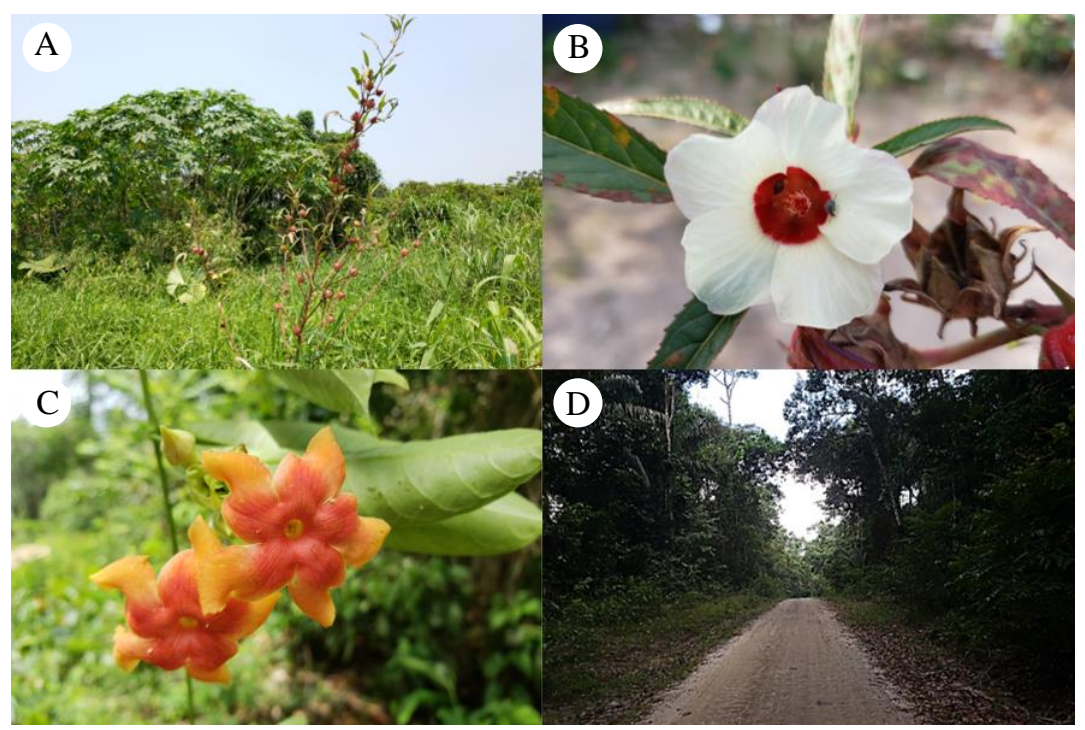

Figure 3. Photographic records of the sampling region: a. Naturalized occurrence of Hibiscus sabdariffa L. in a disturbed area; b. Hibiscus sabdariffa L. flower with white petals, fleshy chalice involving the fruit, and leaves (Almeida, C. et al., p. 97); c. Mandevilla sp. nov. axillary, puberulent inflorescence (Almeida, C. \& Castro, A. 205 (INPA); d. Place of collection of the new

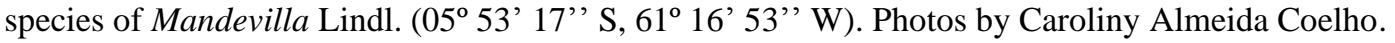

In the southern area of the Amazon, fields or scrublands are supposedly growing due to a shift in a 200-kilometer-long segment of the Madeira river to the east. This shift occurred a few years ago from a rearrangement of tectonic faults, changing the location of many of the right bank tributaries (Pivetta, 2011). A new type of vegetation emerges over the old beds of these rivers that were buried with sandy sediments, forming fields and scrublands in the Amazon (Pivetta, 2011).

Our data indicate that the first botanical collections in Manicoré were performed in the early twentieth century, precisely in 1923 ( $\mathrm{n}=1$ collection). By 1970, only 32 species had been collected. In this sense, collection expeditions were amplified from the 70's $(\mathrm{n}=47)$ and 80's $(\mathrm{n}=152)$, decreased in the 90's $(\mathrm{n}=1)$, and expanded again in $2007(\mathrm{n}=209)$ and $2018(\mathrm{n}=176)$. Although the 1980s accounted for the largest collection peak of the last century, no study addressed the floristic composition of the municipality. However, these collections constituted works of greater geographical scope, such as the RADAMBRASIL Project.

Despite collection efforts made in the last century, about $70 \%$ of the species presented in this study were collected in the $21^{\text {st }}$ century, with two major collection peaks in 2007 and 2018. Botanical collections performed in 2007 are part of an ethnobotanical survey (Junqueira, 2008). Collection peaks between 2018 and 2019 are the result of 28 botanical expeditions made for this checklist, which accounted for $25.2 \%$ of the total species listed for Manicoré, making the sites of these collections become the areas with the highest record of angiosperm species for the municipality (Figure 4).

Collection gaps, mainly in the central region of Manicore, indicate the lack of research in much of the municipality. For botanical collections, forest areas with easier access are generally better researched (Hopkins, 2007). Collection records for Manicoré, are concentrated near the urban area, on the banks of the Madeira river and on part of the BR 319 Highway, where the main communities of the municipality are located (Figure 4).

In the expeditions carried out in this study, we collected 240 individuals from 63 families, 145 genera, and 202 species (Figure 4). In just $90 \mathrm{~h}$ of field sampling, besides finding evidence to prove the naturalization of Hibiscus sabdariffa (Coelho \& Amorim, 2019), it was also possible to identify a new species of Mandevilla Lindl. (Apocynaceae) endemic to the southern Amazon region (Coelho et al., in press) (Figure 3c). Therefore, we estimate that the areas that have collection gaps in Manicoré also have the potential to harbor new taxa for science.

During floristic surveys, it is common to discover new occurrences (Ivanauskas, Monteiro \& Rodrigues, 2004; Lopes, Ribeiro, Rodrigues, Cabral \& Silva, 2014) and occasionally new taxa for science (Baitello, Arzolla \& Vilela, 2017). This shows part of the advances made in recent years, but mainly indicates how much the Brazilian flora still needs to be known (Peixoto \& Morim, 2013). 


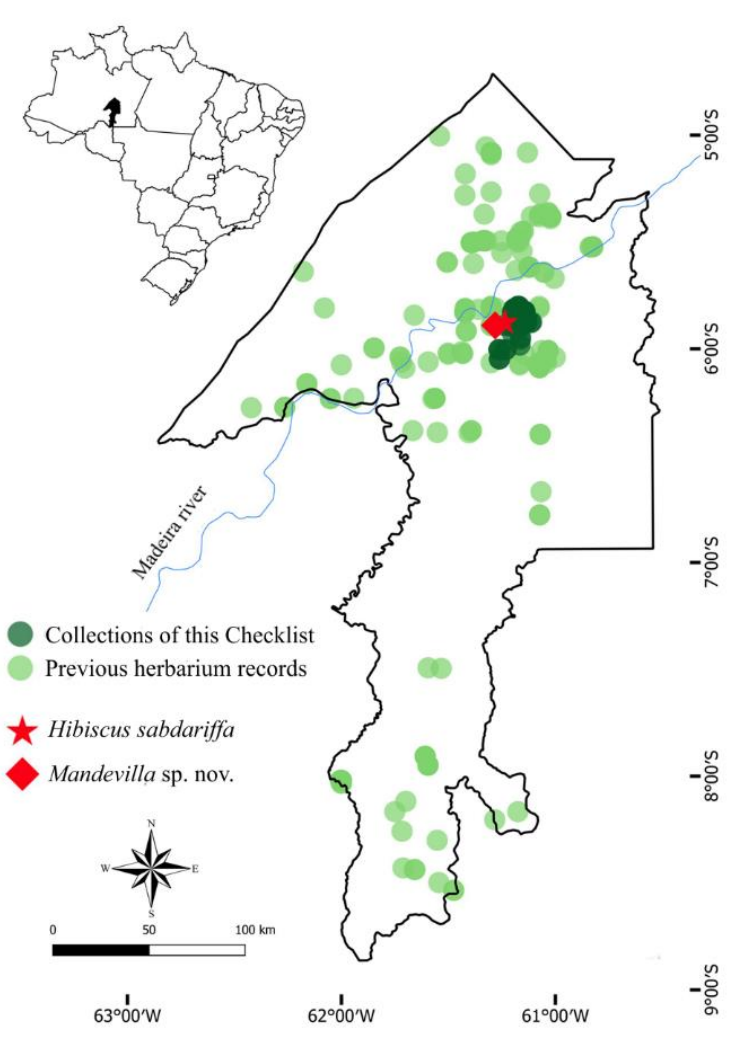

Figure 4. Distribution of angiosperm species collections in Manicoré-AM.

The new species of Mandevilla was found on the edge of a newly opened road in a primary forest fragment located in the municipality countryside (Figure 3d). This new species is described as Mandevilla manicorensis C.A. Coelho, B.S. Amorim \& J.F. Morales (Coelho et al., in press.). The species shows foliate bracts and hypocrateriform corolla and is part of the Exothostemon group. In this group, twelve species have hypocrateriform corolla and only four species have foliate bracts. Easier road access contributed to this botanical discovery. However, ease of access may compromise local flora in the future, with the expansion of the municipality urban center (Salles, Grigio \& Silva, 2013).

Among the cities south of the Madeira river, Beruri, Tapauá, Apuí, Novo Aripuanã, and Manicoré are, respectively, those with the largest gaps in angiosperm collections (Coelho \& Amorim, unpublished data), which could be indicative of low richness (Figure 5). Nonetheless, collection gaps in these cities are due to low sampling effort rather than absence of species, as we can find a large number of species in neighboring areas of similar forests. Thus, we can affirm that these areas are subsampled and consequently prone to contain species not yet identified or described (Hopkins, 2007; ter Steege et al., 2016).

Comparing Manicoré to neighboring municipalities, some factors help to understand why Humaitá is less subsampled. The municipality of Humaitá has access roads through Amazonas State and Rondônia. In addition, it houses a larger number of higher education institutions, which facilitates research in 'terra firme' and floodplain areas (Campos, Ribeiro, Souza-Junior, Ribeiro-Filho \& Almeida, 2012), Cerrado fields (Martins, Ferreira, Curi, Vitorino \& Silva, 2006), and meadows (Kubitski, 1979). Notwithstanding, although Humaitá and Borba have the largest number of species records among the cities south of the Madeira river, subsampling may occur due to the low proportion of angiosperm species per $10 \mathrm{~km}^{2}$ (Figure 5).

Collection gaps are large for angiosperms, but much larger for other groups such as bryophytes, ferns, lycophytes, gymnosperms, and fungi (Coelho \& Amorim, unpublished data). In this sense, it is necessary to continuously create intensive projects to make local floras (Hopkins, 2007, 2019; Forzza et al., 2010; Lopes et al., 2014). These projects should ensure that new collection data are incorporated into herbariums so that the true biological diversity of these areas can be known and rare species unknown to science can be found (Ribeiro et al., 1994; Hopkins, 2007, 2019; Forzza et al., 2010).

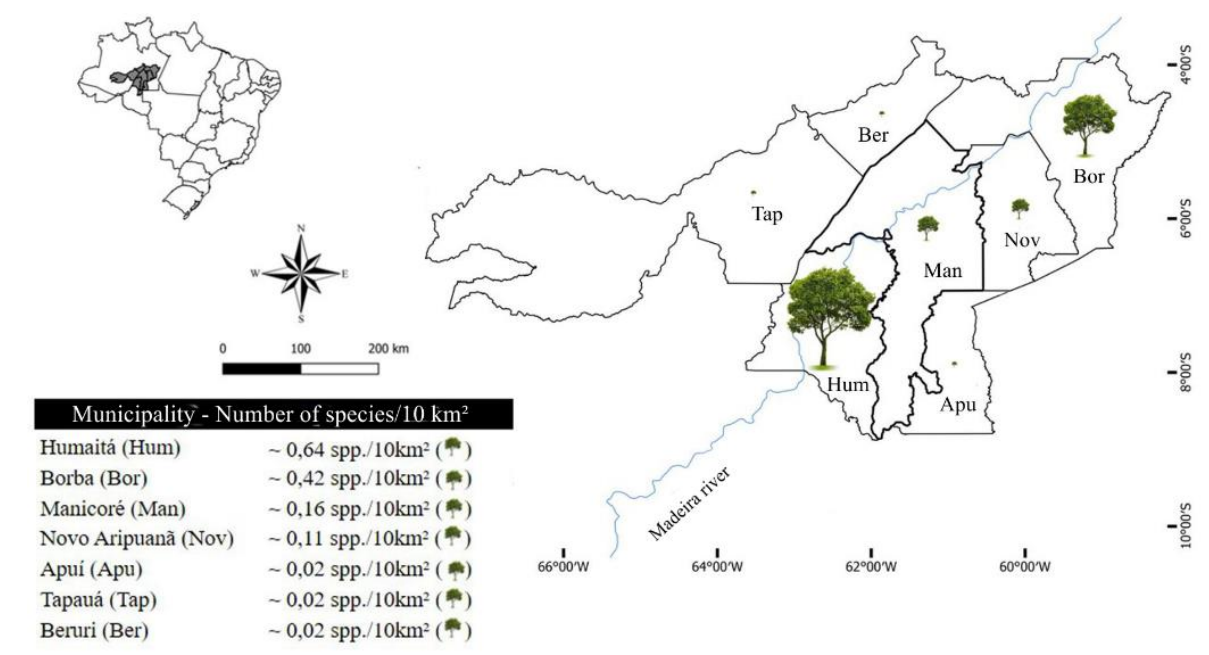

Figure 5: Representation of angiosperm collection records in the cities south of the Madeira river. Tree sizes correspond to the proportion of number of species recorded for each municipality (number of species per ten square kilometers of the municipality). 
Tabela 1: Preliminary angiosperm checklist in an area south of the Madeira River (Manicoré, Amazonas, Brazil). (*) Species not mentioned for Amazonas in Brazil 2020 Flora under construction. (**) Species not mentioned in the Northern region of Brazil 2020 Flora under construction. (***) Species not cited for Brazil in Flora do Brasil 2020 under construction. (\#) Naturalized species for a Brazilian flora. (\#\#) Species cultivated in the Brazilian flora. (!) New species for Brazilian flora. Habit: shrub (ARB), tree (ARV), weed (ERV), liana (LIA), palm tree (PAL), shrub (SUB) and creeper (TRE). Phytogeographic domain: Anthropic Area (AA) Amazon (AM), Caatinga (CAA), Cerrado (CE), Atlantic Forest (MA), Pampas (PAM), Pantanal (PAT) and Unknown (DES).

\begin{tabular}{|c|c|c|c|}
\hline Scientific name & Habit & $\begin{array}{c}\text { Phytogeographic } \\
\text { domain }\end{array}$ & Voucher \\
\hline \multicolumn{4}{|l|}{ Acanthaceae } \\
\hline Asystasia gangetica (L.) T.Anderson**\# & $\mathrm{ARB}$ & MA & Almeida, C. \& Castro, A. 133 (INPA) \\
\hline Justicia comata (L.) & ARB & $\mathrm{AM}, \mathrm{CE}, \mathrm{MA}$ & Mendes, R.F. et al. 2 (EAFM) \\
\hline Justicia pectoralis Jacq & $\mathrm{ARB}$ & $\mathrm{AM}, \mathrm{CE}$ & Mendes, R.F. et al. 15 (EAFM) \\
\hline Mendoncia velloziana Mart. & TRE & AM, MA & Almeida, C. \& Castro, A. 246 (INPA) \\
\hline Pachystachys coccinea (Aubl.) Nees & $\mathrm{ARB}$ & $\mathrm{AM}$ & Almeida, C. \& Castro, A. 145 (INPA) \\
\hline Pranceacanthus coccineus Wassh & ERV & $\mathrm{AM}, \mathrm{CE}$ & Almeida, C. \& Castro, A.109 (INPA) \\
\hline \multicolumn{4}{|l|}{ Achariaceae } \\
\hline Lindackeria paludosa (Benth.) Gilg. & ARV & $\mathrm{AM}$ & $\begin{array}{l}\text { Junqueira, A.B. \& Barros, R.N.S. } 353 \\
\text { (EAFM) }\end{array}$ \\
\hline \multicolumn{4}{|l|}{ Amaranthaceae } \\
\hline Achyranthes aspera $\mathrm{L}$. & ERV & AM, CAA, CE, MA & Almeida, C. \& Castro, A. 241 (INPA) \\
\hline Alternanthera dentata (Moench) Stuchlik ex R. E. Fr. & SUB & $\begin{array}{l}\text { AM, CAA, CE, MA } \\
\text { AM, CAA, CE, MA, }\end{array}$ & Mendes, R.F. et al. 49 (EAFM) \\
\hline Alternanthera tenella Colla & ERV & PAM, PAT & Almeida, C. \& Castro, A. 22 (INPA) \\
\hline Celosia argentea $\mathrm{L} . \#$ & $\mathrm{ARB}$ & AM, CAA, CE, MA & Almeida, C. \& Castro, A. 40 (INPA) \\
\hline Dysphania ambrosioides (L.) Mosyakin \& Clemants & SUB & AM, CAA, CE, MA & Mendes, R.F. et al. 29 (EAFM) \\
\hline \multicolumn{4}{|l|}{ Amaryllidaceae } \\
\hline Hippeastrum puniceum (Lam.) Kuntze & ERV & $\begin{array}{l}\text { AM, CAA, CE, MA, } \\
\text { PAM, PAT }\end{array}$ & Almeida, C.\& Castro, A. 144 (INPA) \\
\hline \multicolumn{4}{|l|}{ Anacardiaceae } \\
\hline Anacardium giganteum W.Hancock ex Engl. & ARV & $\begin{array}{c}\mathrm{AM} \\
\mathrm{AM}, \mathrm{CAA}, \mathrm{CE}, \mathrm{MA},\end{array}$ & Amoêdo, S.C. et al. 57 (EAFM) \\
\hline Anacardium occidentale L. & $\mathrm{ARB}$ & PAM, PAT & $\begin{array}{l}\text { Almeida, C. et al. } 93 \text { (INPA) } \\
\text { Junqueira, A.B. \& Souza, S.A. } 696\end{array}$ \\
\hline Astronium lecointei Ducke. & ARV & $\begin{array}{c}\mathrm{AM} \\
\mathrm{AM}, \mathrm{CAA}, \mathrm{CE}, \mathrm{MA},\end{array}$ & $\begin{array}{l}\text { (EAFM) } \\
\text { Junqueira, A.B. \& Souza, S.A. } 615\end{array}$ \\
\hline Mangifera indica Wall. \#\# & ARV & PAM, PAT & $\begin{array}{l}\text { (EAFM) } \\
\text { Junqueira, A.B. \& Neto, R.F. } 430\end{array}$ \\
\hline Spondias mombin L. & ARV & $\begin{array}{c}\text { AM, CE, MA } \\
\text { AM, CAA, CE, MA, }\end{array}$ & $(\mathrm{EAFM})$ \\
\hline Tapirira guianensis Aubl. & ARV & PAT & $\begin{array}{l}\text { Junqueira, A.B. } 441 \text { (INPA) } \\
\text { Junqueira, A.B. \& Barros, R.N.S. } 373\end{array}$ \\
\hline Thyrsodium spruceanum Benth. & ARV & AM, MA & (INPA) \\
\hline \multicolumn{4}{|l|}{ Annonaceae } \\
\hline Anaxagorea brevipes Benth. & ARV & $\mathrm{AM}$ & Ferreira, C.A.C. 5763 (INPA) \\
\hline Annona annonoides (R.E.Fr.) Maas \& Westra & ARV & $\mathrm{AM}$ & Junqueira, A.B. 468 (INPA) \\
\hline Annona cuspidata (Mart.) H.Rainer & ARV & $\mathrm{AM}$ & Junqueira, A.B. 248 (INPA) \\
\hline Annona excellens R.E.Fr. & ARV & $\mathrm{AM}$ & Ferreira, C.A.C. 5511 (INPA) \\
\hline Annona exsucca DC. & ARV & $\mathrm{AM}$ & $\begin{array}{l}\text { Junqueira, A.B. } 215 \text { (INPA) } \\
\text { Junqueira, A.B. \& Souza, S.A. } 667\end{array}$ \\
\hline Annona foetida Mart. & ARV & $\mathrm{AM}$ & $(\mathrm{EAFM})$ \\
\hline Annona montana Macfad. & ARV & AM, CE, MA, PAT & Viana, G.P. 92 (INPA) \\
\hline Annona muricata L. \#\# & $\mathrm{ARB}$ & DESC & $\begin{array}{l}\text { Mendes, R.F. et al. } 48 \text { (EAFM) } \\
\text { Junqueira, A.B. \& Neto, R.F. } 564\end{array}$ \\
\hline Diclinanona calycina (Diels) R.E.Fr. & ARV & $\mathrm{AM}$ & $\begin{array}{l}\text { (EAFM) } \\
\text { Junqueira, A.B. \& Souza, S.A. } 669\end{array}$ \\
\hline Ephedranthus amazonicus R.E.Fr. & ARV & $\mathrm{AM}$ & (EAFM) \\
\hline Guatteria foliosa Benth. & ARV & $\mathrm{AM}$ & $($ EAFM) \\
\hline Guatteria punctata (Aubl.) R.A.Howard & $\mathrm{ARB}$ & $\mathrm{AM}$ & Almeida, C. \& Castro, A. 239 (INPA) \\
\hline
\end{tabular}




\begin{tabular}{|c|c|c|c|}
\hline Scientific name & Habit & $\begin{array}{l}\text { Phytogeographic } \\
\text { domain }\end{array}$ & Voucher \\
\hline Guatteria scytophylla Diels. & ARV & AM & $\begin{array}{l}\text { Junqueira, A.B. \& Barros, R.N.S. } 352 \\
\text { (EAFM) }\end{array}$ \\
\hline Oxandra riedeliana R.E.Fr. & ARB & AM & Albernaz, A.L.K.M. 180 (INPA) \\
\hline Pseudoxandra lucida R.E.Fr. & ARV & $\mathrm{AM}$ & Ferreira, C.A.C. 5538 (INPA) \\
\hline Unonopsis stipitata Diels & ARV & AM & Ferreira, C.A.C. 5768 (INPA) \\
\hline Xylopia aromatica (Lam.) Mart. & $\mathrm{ARB}$ & $\mathrm{AM}, \mathrm{CE}$ & Ferreira, C.A.C. 5606 (INPA) \\
\hline Xylopia nitida Dunal & ARV & AM & Albernaz, A.L.K.M. 176 (INPA) \\
\hline \multicolumn{4}{|l|}{ Apocynaceae } \\
\hline Allamanda carthatica $\mathrm{L}$. & TRE & AM, CE, MA & Almeida, C. et al. 44 (INPA) \\
\hline Allamanda nobilis T.Moore & TRE & $\mathrm{AM}$ & Almeida, C. \& Castro, A. 73 (INPA) \\
\hline Ambelania acida Aubl. & ARV & AM & Almeida, C. et al. 86 (INPA) \\
\hline Aspidosperma excelsum Benth. & $\mathrm{ARB}$ & $\mathrm{AM}, \mathrm{CE}$ & Ferreira, C.A.C. 5566 (INPA) \\
\hline Barjonia cymosa E.Fourn. ** & SUB & $\mathrm{AM}, \mathrm{CE}$ & Lima, L.C.P. 655 (HUEFS) \\
\hline Ditassa franciscoi (Morillo) Liede & SUB & $\mathrm{AM}$ & Ferreira, C.A.C. 5577 (INPA) \\
\hline Galactophora calycina (Huber) Woodson & ARB & AM & Ferreira, C.A.C. 5798 (INPA) \\
\hline Hancornia speciosa Gomes & $\mathrm{ARB}$ & AM, CAA, CE, MA & Silveira, A.L.P. 289 (UEC) \\
\hline Himatanthus articulatus (Vahl) Woodson. & ARV & $\mathrm{AM}, \mathrm{CE}$ & Almeida, C. et al. 99 (INPA) \\
\hline Himatanthus obovatus (Müll.Arg.) Woodson & ARV & AM, CAA, CE & Lima, L.C.P. 653 (HUEFS) \\
\hline Himatanthus semilunatus Markgr. & $\mathrm{ARB}$ & AM & Ferreira, C.A.C. 5514 (INPA) \\
\hline Himatanthus revolutus (Huber) Spina \& Kinoshita & ARV & $\mathrm{AM}$ & Junqueira, A.B.; Souza, S.A 593 (EAFM) \\
\hline Lacmellea gracilis (Müll.Arg.) Markgr & ARV & AM & Almeida, C. et al. 96 (INPA) \\
\hline Malouetia duckei Markgr. & ARB & $\mathrm{AM}$ & Ferreira, C.A.C. 5542 (INPA) \\
\hline Malouetia tamaquarina (Aubl.) A.DC. & $\mathrm{ARB}$ & AM & Ferreira, C.A.C. 5559 (INPA) \\
\hline Mandevilla sp. nov.! & TRE & & Almeida, C. \& Castro, A. 205 (INPA) \\
\hline $\begin{array}{l}\text { Mandevilla hirsuta (A.Rich.) K.Schum. } \\
\text { Mandevilla scabra (Hoffmanns.ex Roem.\& Schult.) }\end{array}$ & TRE & AM, CAA, CE, MA & Almeida, C. \& Castro, A. 231 (INPA) \\
\hline K.Schum. & TRE & AM, CAA, CE, MA & Lima, L.C.P. 651 (HUEFS) \\
\hline Mandevilla tenuifolia (J.C.Mikan) Woodson & ERV & AM, CAA, CE, MA & Ferreira, C.A.C. 5580 (INPA) \\
\hline Mesechites trifidus (Jacq.) Múll.Arg. & $\mathrm{ARB}$ & AM, CE, PAT & Ferreira, C.A.C. 5779 (INPA) \\
\hline Nephradenia linearis Benth. ex E.Fourn. & ERV & $\mathrm{AM}, \mathrm{CE}$ & Ferreira, C.A.C. 5807 (INPA) \\
\hline Nephradenia reflexa Malme.** & ERV & AM & Ferreira, C.A.C. 5577 (HRCB) \\
\hline Odontadenia nitida (Vahl) Müll.Arg. & TRE & $\mathrm{AM}, \mathrm{CE}$ & $\begin{array}{l}\text { Almeida, C. \& Castro, A. } 151 \text { (INPA) } \\
\text { Junaueira A B \& Souza S A } 678\end{array}$ \\
\hline Rauvolfia sprucei Müll.Arg. & ARV & AM & (EAFM) \\
\hline Spongiosperma grandiflorum (Huber) Zarucchi* & $\mathrm{ARB}$ & AM & $\begin{array}{l}\text { Ferreira, C.A.C. } 5556 \text { (INPA) } \\
\text { Junqueira, A.B. \& Souza, S.A. } 631\end{array}$ \\
\hline Tabernaemontana cymosa Jacq.* & ARV & $\mathrm{AM}$ & (EAFM) \\
\hline Tabernaemontana heterophylla Vahl & ARB & $\mathrm{AM}$ & Almeida, C. \& Castro, A. 219 (INPA) \\
\hline Tabernaemontana linkii A.DC. & $\mathrm{ARB}$ & AM & Almeida, C. \& Castro, A. 138 (INPA) \\
\hline Tabernaemontana muricata Link ex. Roem. \& Schult. & ARV & AM & Krukoff, B.A 6067 (Usw) \\
\hline Tabernaemontana siphilitica (L.) Leeuwenb. & $\mathrm{ARB}$ & $\mathrm{AM}, \mathrm{CE}$ & Viana, G.P. 60 (INPA) \\
\hline \multicolumn{4}{|l|}{ Araceae } \\
\hline Anthurium gracile (Rudge) Lindl. & ERV & $\mathrm{AM}, \mathrm{CAA}, \mathrm{CE}$ & Carvalho, F.A. de 1918 (INPA) \\
\hline Anthurium gracile (Rudge) Lindl. & ERV & AM, CAA, CE & Almeida, C \& Castro, A. 250 (INPA) \\
\hline Heteropsis linearis A.C. Sm. & TRE & AM & Mota, C.D.A. da 61600 (INPA) \\
\hline Philodendron wittianum Engl. & ERV & $\mathrm{AM}$ & Almeida, C \& Castro, A. 227 (INPA) \\
\hline Xanthosoma striatipes (Kunth \& Bouche) Madison & ERV & $\mathrm{AM}, \mathrm{CE}$ & Ferreira, C.A.C. 5573 (INPA) \\
\hline Araliaceae & & & \\
\hline $\begin{array}{l}\text { Schefflera morototoni (Aubl.) Maguire, Steyerm. \& } \\
\text { Frodin. }\end{array}$ & ARV & $\begin{array}{l}\text { AM, CAA, CE, MA, } \\
\text { PAT }\end{array}$ & $\begin{array}{l}\text { Junqueira, A.B. \& Souza, S.A. } 610 \\
\text { (EAFM) }\end{array}$ \\
\hline \multicolumn{4}{|l|}{ Arecaceae } \\
\hline Astrocaryum acaule Mart. & ERV & AM & Almeida, C. \& Castro, A. 200 (INPA) \\
\hline Astrocaryum aculeatum G.Mey. & ERV & $\mathrm{AM}, \mathrm{CE}$ & Almeida, C. \& Castro, A. 209 (INPA) \\
\hline
\end{tabular}


Table 1: continuation.

\begin{tabular}{|c|c|c|c|}
\hline Scientific name & Habit & $\begin{array}{c}\text { Phytogeographic } \\
\text { domain }\end{array}$ & Voucher \\
\hline Attalea attaleoides (Barb.Rodr.) Wess.Boer. & PAL & $\mathrm{AM}$ & $\begin{array}{l}\text { Junqueira, A.B. \& Neto, R.F } 572 \\
\text { (EAFM) }\end{array}$ \\
\hline Attalea phalerata Mart. ex Spreng. & PAL & $\mathrm{AM}, \mathrm{CE}$ & Junqueira, A.B.; Neto, R.F. 578 (EAFM) \\
\hline $\begin{array}{l}\text { Bactris acanthocarpa Mart. var. trailiana (Barb.Rodr.) } \\
\text { A.J.Hend. }\end{array}$ & ERV & $\mathrm{AM}$ & Henderson, A.J. 298 (INPA) \\
\hline Bactris bifida Mart. & PAL & $\mathrm{AM}$ & $\begin{array}{l}\text { Junqueira, A.B. \& Barros, R.N.S. } 377 \\
\text { (EAFM) }\end{array}$ \\
\hline Bactris concinna Mart. & PAL & $\mathrm{AM}$ & Junqueira, A.B. 253 (INPA) \\
\hline Bactris hirta Mart. & ERV & AM, MA & Emilio, T. 634 (INPA) \\
\hline Bactris killipii Burret & ERV & $\mathrm{AM}$ & Emilio, T. 518 (INPA) \\
\hline Bactris maraja Mart. & PAL & $\mathrm{AM}$ & Junqueira, A.B. 571 (INPA) \\
\hline Bactris simplicifrons Mart. & PAL & $\mathrm{AM}$ & Junqueira, A.B. 568 (INPA) \\
\hline Bactris tomentosa Mart. & PAL & $\mathrm{AM}$ & Junqueira, A.B. 700 (INPA) \\
\hline Desmoncus mitis subsp. leptospadix (Martius) Henderson & PAL & $\mathrm{AM}$ & $\begin{array}{l}\text { Junqueira, A.B. \& Barros, R.N.S. } 340 \\
\text { (EAFM) }\end{array}$ \\
\hline Desmoncus parvulus L.H.Bailey & PAL & $\mathrm{AM}$ & Junqueira, A. B. 216 (NYBG) \\
\hline Desmoncus polyacanthos Mart. & ERV & $\mathrm{AM}, \mathrm{CE}, \mathrm{MA}$ & Almeida, C. \& Castro, A. 206 (INPA) \\
\hline Elaeis oleifera (Kunth) Cortés & ERV & $\mathrm{AM}$ & Silva, G. P. 337 (CEN) \\
\hline Euterpe oleracea Mart.* & PAL & $\mathrm{AM}, \mathrm{CE}$ & Mendes, R.F. et al. 7 (EAFM) \\
\hline Geonoma deversa (Poit.) Kunth & PAL & $\mathrm{AM}$ & Junqueira, A.B. 285 (INPA) \\
\hline Geonoma leptospadix Trail & PAL & $\mathrm{AM}$ & Viana, G.P. 76 (INPA) \\
\hline Geonoma macrostachys Mart. & PAL & $\mathrm{AM}$ & Viana, G.P. 69 (INPA) \\
\hline Geonoma maxima (Poit.) Kunth & PAL & $\mathrm{AM}$ & Almeida, C. et al. 14 (INPA) \\
\hline Oenocarpus minor Mart. & PAL & $\mathrm{AM}$ & Almeida, C. et al. 81 (INPA) \\
\hline Oenocarpus minor Mart. & PAL & $\mathrm{AM}$ & $\begin{array}{l}\text { Junqueira, A.B. \& Souza, J.R. } 252 \\
\text { (EAFM) }\end{array}$ \\
\hline Socratea exorrhiza (Mart.) H.Wendl. & PAL & $\mathrm{AM}$ & Henderson, AJ. 297 (NY) \\
\hline \multicolumn{4}{|l|}{ Aristolochiaceae } \\
\hline Aristolochia sprucei Mast. & TRE & $\mathrm{AM}$ & Almeida, C. \& Castro, A. 158 (INPA) \\
\hline \multicolumn{4}{|l|}{ Asteraceae } \\
\hline Acmella oleracea (L.) R.K.Jansen\# & ERV & AM, MA & Mendes, R.F. et al. 1 (EAFM) \\
\hline Ayapana amygdalina (Lam.) R.M. King \& H. Rob. & ERV & AM, CAA, CE, MA & Giacomin, L.L. et al. 1917 (UNOP) \\
\hline Bidens cynapiifolia Kunth. & $\mathrm{ARB}$ & $\mathrm{AM}, \mathrm{CE}, \mathrm{MA}$ & Mendes, R.F. et al. 35 (EAFM) \\
\hline Chromolaena laevigata (Lam.) R.M. King \& H. Rob. & $\mathrm{ARB}$ & $\begin{array}{l}\text { AM, CAA, CE, MA, } \\
\text { PAM }\end{array}$ & Giacomin, L.L. et al. 1927 (UNOP) \\
\hline Eclipta prostrata (L.) L. & ERV & $\begin{array}{l}\text { AM, CAA, CE, MA, } \\
\text { PAM, PAT }\end{array}$ & Almeida, C. \& Castro, A. 191 (INPA) \\
\hline Emilia fosbergii Nicolson Paratype. & ERV & $\begin{array}{l}\text { AM, CAA, CE, MA, } \\
\text { PAM, PAT }\end{array}$ & Krukoff, B.A. 6066 (MO) \\
\hline Ichthyothere terminalis (Spreng.) S.F.Blake & SUB & AM, CAA, CE & Almeida, C. et al. 60 (INPA) \\
\hline Mikania psilostachya DC. & LIA & AM, CAA, CE, MA & Ferreira, C.A.C. 5521 (INPA) \\
\hline $\begin{array}{l}\text { Gymnanthemum amygdalinum (Delile) Sch.Bip. ex } \\
\text { Walp. *\#\# }\end{array}$ & ARB & $\mathrm{AM}, \mathrm{CE}, \mathrm{MA}$ & Mendes, R.F. et al. 13 (EAFM) \\
\hline Tithonia diversifolia (Hemsl.) A.Gray\# & ERV & $\mathrm{AM}, \mathrm{CE}, \mathrm{MA}$ & Almeida, C. \& Castro, A. 27 (INPA) \\
\hline Vernonanthura brasiliana (L.) H.Rob. * & ARV & $\mathrm{AM}, \mathrm{CAA}, \mathrm{CE}$ & $\begin{array}{l}\text { Junqueira, A.B. \& Barros, R.N.S. } 288 \\
\text { (EAFM) }\end{array}$ \\
\hline Vernonanthura patens (Kunth) H.Rob & ARB & AM, MA & Almeida, C. \& Castro, A. 103 (INPA) \\
\hline Wedelia rudis (Baker) H.Rob. & SUB & $\mathrm{AM}$ & Almeida, C. \& Castro, A. 193 (INPA) \\
\hline \multicolumn{4}{|l|}{ Bignoniaceae } \\
\hline Amphilophium laeve (Sandwith) L.G.Lohmann & $\mathrm{ARB}$ & $\mathrm{AM}$ & Ferreira, C.A.C. 5808 (INPA) \\
\hline Amphilophium magnoliifolium (Kunth) L.G.Lohmann & LIA & $\mathrm{AM}$ & Ferreira, C.A.C. 5539 (NYBG) \\
\hline Amphilophium paniculatum (L.) Kunth & LIA & $\begin{array}{l}\text { AM, CAA, CE, MA, } \\
\text { PAT }\end{array}$ & Almeida, C. \& Castro, A. 173 (INPA) \\
\hline Anemopaegma foetidum Bureau \& K.Schum. & TRE & $\mathrm{AM}$ & $\begin{array}{l}\text { Almeida, C. \& Turma CB15_ME01 } 185 \\
\text { (INPA) }\end{array}$ \\
\hline Bignonia cf lilacina (A.H.Gentry) L.G.Lohmann & TRE & $\mathrm{AM}$ & Almeida, C. \& Castro, A. 89 (INPA) \\
\hline
\end{tabular}




\begin{tabular}{|c|c|c|c|}
\hline Scientific name & Habit & $\begin{array}{c}\text { Phytogeographic } \\
\text { domain }\end{array}$ & Voucher \\
\hline Crescentia cujete L. \#\# & ARV & AM, MA & Krukoff, B. A. 6036 (NYBG) \\
\hline Fridericia bracteolata (DC.) L.G.Lohmann & LIA & $\mathrm{AM}$ & Albernaz, A.L.K.M. 178 (INPA) \\
\hline Fridericia chica (Bonpl.) L.G.Lohmann & LIA & $\begin{array}{l}\text { AM, CAA, CE, MA, } \\
\text { PAM, PAT }\end{array}$ & $\begin{array}{l}\text { Junqueira, A.B. \& Souza, S.A. } 707 \\
\text { (EAFM) }\end{array}$ \\
\hline Fridericia florida (DC.) L.G.Lohmann & LIA & AM, CE, MA & Almeida C. \& Castro, A. 161 (INPA) \\
\hline Fridericia platyphylla (Cham.) L.G.Lohmann & ARB & $\begin{array}{l}\text { AM, CAA, CE, MA, } \\
\text { PAT }\end{array}$ & Almeida C. \& Castro, A. 167 (INPA) \\
\hline Handroanthus impetiginosus (Mart. ex DC.) Mattos* & ARV & $\begin{array}{l}\text { AM, CAA, CE, MA, } \\
\text { PAT }\end{array}$ & Amoêdo, S.C. et al. 39 (EAFM) \\
\hline Jacaranda campinae A.H.Gentry \& Morawetz & ARB & $\mathrm{AM}$ & Ferreira, C.A.C. $5816(\mathrm{NY})$ \\
\hline Lundia densiflora DC. & LIA & AM, CAA, CE, MA & $\begin{array}{l}\text { Junqueira, A.B. \& Souza, S.A } 595 \\
\text { (EAFM) }\end{array}$ \\
\hline Pleonotoma melioides (S.Moore) A.H.Gentry & $\mathrm{ARB}$ & $\mathrm{AM}, \mathrm{CE}, \mathrm{MA}$ & Almeida, C. \& Castro, A. 118 (INPA) \\
\hline Pleonotoma pavettiflora Sandwith* & LIA & AM, CE, PAT & Lombardi, J.A. 10095 (INPA) \\
\hline Pyrostegia venusta (Ker Gawl.) Miers & ARB & $\begin{array}{l}\text { AM, CAA, CE, MA, } \\
\text { PAM, PAT }\end{array}$ & Almeida, C. \& Castro, A. 53 (INPA) \\
\hline Sparattosperma leucanthum (Vell.) K.Schum. & $\mathrm{ARB}$ & $\begin{array}{l}\text { AM, CAA, CE, MA, } \\
\text { PAT }\end{array}$ & Almeida, C. \& Castro, A. 234 (INPA) \\
\hline \multicolumn{4}{|l|}{ Bixaceae } \\
\hline Bixa orellana $L$. & ARB & AM, CE, MA & Almeida, C. et al. 82 (INPA) \\
\hline Cochlospermum orinocense (Kunth) Steud. & $\mathrm{ARB}$ & $\mathrm{AM}$ & Almeida, C. \& Castro, A. 100 (INPA) \\
\hline \multicolumn{4}{|l|}{ Boraginaceae } \\
\hline Cordia decipiens I.M.Johnst. & LIA & $\mathrm{AM}$ & Krukoff, B. A. 6048 (NYBG) \\
\hline Cordia scabrifolia A.DC. & ARV & $\mathrm{AM}$ & $\begin{array}{l}\text { Junqueira, A.B. \& Barros, R.N.S. } 347 \\
\text { (EAFM) }\end{array}$ \\
\hline Cordia sellowiana Cham.* & ARB & AM, CAA, CE, MA & Almeida, C \& Castro, A. 221 (INPA) \\
\hline Cordia sprucei Mez. & ARV & $\mathrm{AM}$ & $\begin{array}{l}\text { Junqueira, A.B. \& Barros, R.N.S. } 374 \\
\text { (EAFM) }\end{array}$ \\
\hline \multicolumn{4}{|l|}{ Bromeliaceae } \\
\hline Aechmea cf angustifolia Poepp. \& Endl. & ERV & $\mathrm{AM}$ & Almeida, C. \& Castro, A. 126 (INPA) \\
\hline Aechmea melinonii Hook. & ERV & $\mathrm{AM}$ & Ferreira, C.A.C. 5787 (INPA) \\
\hline Aechmea mertensii (G.Mey.) Schult. \& Schult.f. & ERV & AM, MA & Almeida, C. \& Castro, A. 110 (INPA) \\
\hline Ananas ananassoides (Baker) L.B.Sm. & ERV & AM, CAA, CE, MA & Almeida, C. et al. 94 (INPA) \\
\hline Ananas lucidus Mill. & ERV & AM, MA & Almeida, C. et al. 95 (INPA) \\
\hline Araeococcus micranthus Brongn. & ERV & AM, MA & Carvalho, F.A. de 1951 (INPA) \\
\hline \multicolumn{4}{|l|}{ Burseraceae } \\
\hline Dacryodes paraensis Cuatrec* & ARV & $\mathrm{AM}, \mathrm{CE}$ & Carvalho, F.A. 1927 (INPA) \\
\hline Protium giganteum Engl. var. gigateum & ARV & $\mathrm{AM}$ & Mota, C.D.A. da 616006 (INPA) \\
\hline Protium glabrescens Swart & ARV & $\mathrm{AM}$ & Viana, G.P. 93 (INPA) \\
\hline Protium goudotianum (Tul.) Byng \& Christenh. & ARV & DESC & Krukoff, B.A. 6066 (MO) \\
\hline Protium grandifolium Engl. & ARV & $\mathrm{AM}$ & Mota, C.D.A. da 61462 (INPA) \\
\hline Protium heptaphyllum (Aubl.) Marchand & ARV & AM, CAA, CE, MA & Amoêdo, S.C. et al. 38 (EAFM) \\
\hline $\begin{array}{l}\text { Protium heptaphyllum (Aubl.) Marchand ssp. ulei (Swart) } \\
\text { Daly }\end{array}$ & ARV & $\mathrm{AM}$ & Carvalho, F.A. de 1915 (INPA) \\
\hline Protium nitidifolium (Cuatrec.) Daly. & ARV & $\mathrm{AM}$ & $\begin{array}{l}\text { Junqueira, A.B. \& Neto, R.F. } 482 \\
\text { (EAFM) }\end{array}$ \\
\hline Protium paniculatum Engl. var. riedelianum (Engl.) Daly & ARB & $\mathrm{AM}$ & Mota, C.D.A. da 61664 (INPA) \\
\hline Protium picramnioides Byng \& Christenh. & ARV & $\mathrm{AM}$ & $\begin{array}{l}\text { Junqueira, A.B. \& Neto, R.F. } 399 \\
\text { (EAFM) }\end{array}$ \\
\hline Protium rhoifolium (Benth.) Byng \& Christenh. & ARV & $\mathrm{AM}$ & $\begin{array}{l}\text { Junqueira, A.B. \& Souza, S.A. } 682 \\
\text { (EAFM) }\end{array}$ \\
\hline Protium robustum (Swart) D.M.Porter. & ARV & $\mathrm{AM}$ & $\begin{array}{l}\text { Junqueira, A.B. \& Barros, R.N.S. } 332 \\
\text { (EAFM) }\end{array}$ \\
\hline Protium sagotianum Marchand & ARV & $\mathrm{AM}$ & Mota, C.D.A. da 61667 (INPA) \\
\hline Protium cf spruceanum (Benth.) Engl. & $\mathrm{ARB}$ & $\mathrm{AM}, \mathrm{CE}, \mathrm{MA}$ & Almeida, C. \& Castro, A. 208 (INPA) \\
\hline Trattinnickia burserifolia Mart. & ARB & $\mathrm{AM}$ & Ferreira, C.A.C. 5571 (INPA) \\
\hline
\end{tabular}


Table 1: continuation.

\begin{tabular}{|c|c|c|c|}
\hline Scientific name & Habit & $\begin{array}{l}\text { Phytogeographic } \\
\text { domain }\end{array}$ & Voucher \\
\hline Trattinnickia glaziovii Swart. & ARV & $\mathrm{AM}$ & $\begin{array}{l}\text { Junqueira, A.B. \& Neto, R.F. } 524 \\
\text { (EAFM) }\end{array}$ \\
\hline Trattinnickia peruviana Loes. & ARV & $\mathrm{AM}$ & $\begin{array}{l}\text { Junqueira, A.B. \& Souza, S.A. } 602 \\
\text { (EAFM) }\end{array}$ \\
\hline Trattinnickia rhoifolia Willd. & ARV & AM & $\begin{array}{l}\text { Junqueira, A.B. \& Souza, S.A. } 591 \\
\text { (EAFM) }\end{array}$ \\
\hline \multicolumn{4}{|l|}{ Calophyllaceae } \\
\hline Caraipa densifolia subsp. rondoniana Kubitzki & ARV & $\mathrm{AM}, \mathrm{CE}$ & Silveira, A.L.P. 291 (RON) \\
\hline Caraipa grandifolia Mart. & ARV & $\mathrm{AM}$ & Almeida, T.E. 3611 (INPA) \\
\hline Caraipa savannarum Kubitzki & ARB & $\mathrm{AM}$ & Mota, C.D.A. da 61616 (INPA) \\
\hline \multicolumn{4}{|l|}{ Cannabaceae } \\
\hline Celtis iguanaea (Jacq.) Schult. & ARV & $\begin{array}{l}\text { AM, CAA, CE, MA, } \\
\text { PAM, PAT } \\
\text { AM, CAA, CE, MA, }\end{array}$ & $\begin{array}{l}\text { Junqueira, A.B. \& Barros, R.N.S. } 327 \\
\text { (EAFM) }\end{array}$ \\
\hline Trema micrantha (L.) Blume & ARV & PAM, PAT & Junqueira, A.B. 414 (INPA) \\
\hline \multicolumn{4}{|l|}{ Capparaceae } \\
\hline Crateva tapia $\mathrm{L}$. & ARV & $\begin{array}{c}\text { AM, CAA, CE, MA, } \\
\text { PAT }\end{array}$ & Viana, G.P. 111 (INPA) \\
\hline \multicolumn{4}{|l|}{ Caricaceae } \\
\hline $\begin{array}{l}\text { Carica papaya L.\# } \\
\text { Jacaratia digitata (Poepp. \& Endl.) Solms }\end{array}$ & ARB & $\begin{array}{l}\text { AM, CAA, CE, MA, } \\
\text { PAT } \\
\text { AM }\end{array}$ & $\begin{array}{l}\text { Mendes, R.F. et al. } 28 \text { (EAFM) } \\
\text { Junqueira, A.B. \& Barros, R.N.S. } 225 \\
\text { (EAFM) }\end{array}$ \\
\hline \multicolumn{4}{|l|}{ Caryocaraceae } \\
\hline $\begin{array}{l}\text { Caryocar glabrum (Aubl.) Pers. } \\
\text { Caryocar villosum (Aubl.) Pers. }\end{array}$ & $\begin{array}{l}\text { ARV } \\
\text { ARV }\end{array}$ & $\begin{array}{l}\mathrm{AM} \\
\mathrm{AM}\end{array}$ & $\begin{array}{l}\text { Amoêdo, S.C. et al. } 37 \text { (EAFM) } \\
\text { Junqueira, A.B. \& Neto, R.F. } 582 \\
\text { (EAFM) }\end{array}$ \\
\hline \multicolumn{4}{|l|}{ Celastraceae } \\
\hline Cheiloclinium cognatum (Miers) A.C.Sm. & ARV & AM, CE, MA, PAT & $\begin{array}{l}\text { Junqueira, A.B. \& Souza, J.R. } 255 \\
\text { (EAFM) }\end{array}$ \\
\hline Cheiloclinium obtusum A.C.Sm. & LIA & $\begin{array}{c}\text { AM } \\
\text { AM, CAA, CE, MA, }\end{array}$ & Mota, C.D.A. da 61604 (INPA) \\
\hline Hippocratea volubilis $\mathrm{L}$. & TRE & PAT & Ferreira, C.A.C. 5785 (INPA) \\
\hline \multicolumn{4}{|l|}{ Chrysobalanaceae } \\
\hline Acioa guianensis Aubl. & ARV & $\mathrm{AM}$ & Mota, C.D.A. da 61659 (INPA) \\
\hline Couepia chrysocalyx (Poepp. \& Endl.) Benth. ex Hook. f. & ARV & $\mathrm{AM}$ & Viana, G.P. 84 (INPA) \\
\hline Couepia latifolia Standl. & ARV & $\mathrm{AM}$ & Krukoff, B. A. 6005 (NYBG) \\
\hline Couepia paraensis (Mart. \& Zucc.) Benth. ssp. Paraenses & ARV & $\mathrm{AM}, \mathrm{CE}$ & Mota, C.D.A. da 61672 (INPA) \\
\hline Hirtella bullata Benth. & $\mathrm{ARB}$ & $\mathrm{AM}$ & Mota, C.D.A. da 61558 (INPA) \\
\hline Hirtella burchellii (Britton) & ARB & $\mathrm{AM}, \mathrm{CE}$ & Lombardi, J.A. 10100 (HRCB) \\
\hline Hirtella elongata Mart. \& Zucc. & ARV & $\mathrm{AM}$ & (EAFM) \\
\hline Hirtella glandulosa Spreng. & ARV & $\mathrm{AM}, \mathrm{CE}, \mathrm{MA}$ & Silveira, A.L.P. 325 (UEC) \\
\hline Hirtella racemosa Lam. & ARB & AM, CAA, CE, MA & Almeida, C. \& Castro, A. 169 (INPA) \\
\hline Hirtella sprucei Benth. ex Hook.f. ** & ARB & $\mathrm{AM}$ & $\begin{array}{l}\text { Mota, C.D.A. da } 61613 \text { (INPA) } \\
\text { Junqueira. A.B. \& Souza, S.A. } 654\end{array}$ \\
\hline Licania gracilipes Taub. & ARV & AM, CE, MA & $\begin{array}{l}\text { Junqueira, A.B. \& Souza, S.A. } 654 \\
\text { (EAFM) } \\
\text { Junqueira, A.B. \& Neto, R.F. } 549\end{array}$ \\
\hline Licania micrantha Miq. & ARV & $\mathrm{AM}, \mathrm{CE}, \mathrm{MA}$ & (EAFM) \\
\hline $\begin{array}{l}\text { Licania oblongifolia Standl. } \\
\text { Licania octandra (Hoffmanns. ex Roem. \& Schult.) }\end{array}$ & ARB & $\mathrm{AM}$ & Almeida, C. \& Castro, A. 148 (INPA) \\
\hline Kuntze ssp. pallida (Hook.f.) Prance & ARV & AM, CAA, CE, MA & Ferreira, C.A.C. 5732 (INPA) \\
\hline Licania parvifructa Fanshawe \& Maguire & ARV & $\mathrm{AM}$ & Ferreira, C.A.C. 5589 (INPA) \\
\hline Parinari excelsa Sabine & ARV & $\mathrm{AM}, \mathrm{CE}, \mathrm{MA}$ & Viana, G.P. 87 (INPA) \\
\hline \multicolumn{4}{|l|}{ Clusiaceae } \\
\hline Clusia candelabrum Planch. \& Triana & ARV & $\mathrm{AM}$ & Silveira, A.L.P. 12 (UEC) \\
\hline Clusia columnaris Engl. & ARB & $\mathrm{AM}$ & Mota, C.D.A. da 61557 (INPA) \\
\hline
\end{tabular}




\begin{tabular}{|c|c|c|c|}
\hline Scientific name & Habit & $\begin{array}{c}\text { Phytogeographic } \\
\text { domain }\end{array}$ & Voucher \\
\hline Clusia nigrolineata P.F.Stevens* & TRE & AM & Viana, G.P. 94 (INPA) \\
\hline Clusia panapanari (Aubl.) Choisy & $\mathrm{ARB}$ & AM, CAA, MA & Ferreira, C. A.C. 5837 (NYBG) \\
\hline Garcinia benthamiana (Planch. \& Triana) Pipoly & $\mathrm{ARB}$ & $\mathrm{AM}$ & Almeida, C; Castro, A. 240 (INPA) \\
\hline Garcinia gardneriana (Planch. \& Triana) Zappi & ARV & AM, CAA, CE, MA & $\begin{array}{l}\text { Ferreira, C.A.C. } 5778 \text { (NYBG) } \\
\text { Junqueira, A.B. \& Souza, J.R. } 249\end{array}$ \\
\hline Garcinia macrophylla Mart. & ARV & AM, CE, MA & (EAFM) \\
\hline Garcinia madruno (Kunth) Hammel & ARV & AM, CE, MA & Ferreira, C.A.C. 5778 (INPA) \\
\hline Symphonia globulifera L. & ARV & $\mathrm{AM}, \mathrm{CE}, \mathrm{MA}$ & Mota, C.D.A. da 61461 (INPA) \\
\hline \multicolumn{4}{|l|}{ Combretaceae } \\
\hline Combretum indicum (L.) Jongkind*\#\# & ARV & DESC & Almeida, C. \& Castro, A. 174 (INPA) \\
\hline Combretum laxum Jacq. & LIA & AM, CAA, CE, MA & Almeida, C. \& Castro, A. 212 (INPA) \\
\hline Combretum rotundifolium Rich. & $\mathrm{ARB}$ & $\mathrm{AM}$ & Almeida, C \& Castro, A. 123 (INPA) \\
\hline \multicolumn{4}{|l|}{ Connaraceae } \\
\hline Connarus coriaceus $\mathrm{G}$. Schellenb & $\mathrm{ARB}$ & $\mathrm{AM}$ & Cid Ferreira, C.A. 5567 (HRCB) \\
\hline Connarus erianthus Benth. ex Baker & ARB & $\mathrm{AM}$ & Almeida, C \& Castro, A. 117 (INPA) \\
\hline Connarus erianthus var. stipitatus Forero & $\mathrm{ARB}$ & $\mathrm{AM}$ & Krukoff, B. A. 6042 (NYBG) \\
\hline $\begin{array}{l}\text { Connarus ruber Planch. } \\
\text { Connarus ruber (Poepp.) Planch. var. sprucei (Baker) }\end{array}$ & $\mathrm{ARB}$ & $\mathrm{AM}$ & Almeida, C. \& Castro, A. 88 (INPA) \\
\hline Forero & $\mathrm{ARB}$ & $\mathrm{AM}$ & Ferreira, C.A.C. 5572 (INPA) \\
\hline Connarus suberosus Planch* & ARV & CE, PAT & Silveira, A.L.P. 290 (UEC) \\
\hline Rourea amazonica (Baker) Radlk. & LIA & $\mathrm{AM}$ & Viana, G.P. 99 (INPA) \\
\hline Rourea krukovii Steyerm. & $\mathrm{ARB}$ & $\mathrm{AM}$ & Albernaz, A.L.K.M. 177 (INPA) \\
\hline \multicolumn{4}{|l|}{ Convolvulaceae } \\
\hline Calycobolus lanulosus D.F.Austin* & TRE & $\begin{array}{c}\text { AM, CAA, CE } \\
\text { AM, CAA, CE, MA, }\end{array}$ & Giacomin, L.L. et al. 1918 (INPA) \\
\hline Distimake aegyptius (L.) A.R. Simões \& Staples & TRE & PAM, PAT & Almeida, C. \& Castro, A. 52 (INPA) \\
\hline Ipomoea tiliacea (Willd.) Choisy & TRE & AM, MA & Almeida, C. et al. 33 (INPA) \\
\hline Jacquemontia guyanensis (Aubl.) Meisn. & TRE & $\begin{array}{c}\text { AM } \\
\text { AM, CAA, CE, MA, }\end{array}$ & Almeida, C. \& Castro, A. 238 (INPA) \\
\hline Merremia dissecta (Jacq.) Hallier f. & TRE & PAM, PAT & Almeida, C. \& Castro, A. 150 (INPA) \\
\hline Merremia umbellata (L.) Hallier f. & TRE & AM, CAA, CE, MA & Almeida, C. \& Castro, A. 104 (INPA) \\
\hline \multicolumn{4}{|l|}{ Costaceae } \\
\hline $\begin{array}{l}\text { Chamaecostus lanceolatus (Petersen) C.D.Specht \& } \\
\text { D.W.Stev. ssp. Lanceolatus }\end{array}$ & ERV & $\mathrm{AM}$ & Ferreira, C.A.C. 5617 (INPA) \\
\hline Costus arabicus $\mathrm{L}$. & ERV & AM, CE, MA, PAT & Ferreira, C.A.C. 5832 (INPA) \\
\hline Costus spiralis (Jacq.) Roscoe & ERV & AM, CE, MA, PAT & Almeida, C. \& Castro, A. 68 (INPA) \\
\hline \multicolumn{4}{|l|}{ Cucurbitaceae } \\
\hline Cucumis anguria $\mathrm{L}$. & ERV & AM, CAA, CE, MA & Almeida, C. et al. 90 (INPA) \\
\hline Cucurbita maxima Duchesne ex Lam. \#\# & ERV & $\mathrm{AM}, \mathrm{CE}$ & Almeida, C. \& Castro, A. 42 (INPA) \\
\hline Gurania bignoniacea (Poepp. \& Endl.) C. Jeffrey. & ERV & AM, CE, MA, PAT & Krukoff, B. A. 6054 (MO) \\
\hline Gurania eriantha (Poepp. \& Endl.) Cogn. & LIA & AM, CE, MA & Viana, G.P. 103 (INPA) \\
\hline Gurania insolita Cogn. & TRE & AM & Mota, C.D.A. da 61677 (INPA) \\
\hline Gurania lobata (L.) Pruski & TRE & $\mathrm{AM}, \mathrm{CE}, \mathrm{MA}$ & Almeida, C.\& Castro, A. 106 (INPA) \\
\hline Gurania sinuata (Benth.) Cogn. & TRE & $\mathrm{AM}$ & Almeida, C. \& Castro, A. 236 (INPA) \\
\hline \multicolumn{4}{|l|}{ Cyperaceae } \\
\hline Cyperus brevifolius (Rottb) Endl. ex Hassk. & SUB & $\begin{array}{c}\text { AM, CAA, CE, MA, } \\
\text { PAM, PAT } \\
\text { AM, CAA, CE, MA, }\end{array}$ & Almeida, C. \& Castro, A. 56 (INPA) \\
\hline Cyperus luzulae (L.) Retz. & ERV & PAM, PAT & Almeida, C. et al. 43 (INPA) \\
\hline Exochogyne amazonica C.B.Clarke & ERV & $\mathrm{AM}, \mathrm{CE}$ & Ferreira, C.A.C. 5803 (INPA) \\
\hline Hypolytrum pulchrum (Rudge) H.Pfeiff.* & $\mathrm{ARB}$ & AM, MA & Mota, C.D.A. da 61567 (INPA) \\
\hline Lagenocarpus glomerulatus Gilly & ARB & $\mathrm{AM}$ & Mota, C.D.A. da 61562 (INPA) \\
\hline Scleria cyperina Willd. ex Kunth & ERV & PAT & Almeida, C. \& Castro, A. 55 (INPA) \\
\hline
\end{tabular}


Table 1: continuation.

\begin{tabular}{|c|c|c|c|}
\hline Scientific name & Habit & $\begin{array}{c}\text { Phytogeographic } \\
\text { domain }\end{array}$ & Voucher \\
\hline \multicolumn{4}{|l|}{ Dichapetalaceae } \\
\hline Dichapetalum pedunculatum (DC) Baill. & LIA & AM & Almeida, C. \& Castro, A. 210 (INPA) \\
\hline \multicolumn{4}{|l|}{ Dilleniaceae } \\
\hline Davilla lanosa Fraga \& Stehmann & TRE & $\mathrm{AM}$ & Almeida, C. \& Castro, A. 112 (INPA) \\
\hline Davilla nitida (Vahl) Kubitzki & $\mathrm{ARB}$ & AM, CE, MA & Almeida, C. \& Castro, A. 152 (INPA) \\
\hline Doliocarpus brevipedicellatus Garcke & ARB & $\mathrm{AM}, \mathrm{CE}$ & Almeida, C. \& Castro, A. 135 (INPA) \\
\hline \multicolumn{4}{|l|}{ Dioscoreaceae } \\
\hline Dioscorea amaranthoides Presl. & TRE & AM, CAA, CE & Ferreira, CID. 5585 (UB) \\
\hline Dioscorea dodecaneura Vell. & LIA & AM, CAA, CE, MA & Silva, G.P. et al. 341 (TEPB) \\
\hline Dioscorea hassleriana Chodat. ** & LIA & CAA, CE & Glocimar P.S. 332 (CEN) \\
\hline Dioscorea piperifolia Humb. \& Bonpl. ex Willd. & LIA & AM, CAA, CE, MA & Glocimar P.S. 343 (CEN) \\
\hline \multicolumn{4}{|l|}{ Eriocaulaceae } \\
\hline Comanthera reflexa (Gleason) L.R.Parra \& Giul. & ERV & AM & Carvalho, F.A. de 1908 (INPA) \\
\hline $\begin{array}{l}\text { Comanthera xeranthemoides (Bong.) L.R.Parra \& Giul. } \\
\text { Comanthera xeranthemoides (Bong.) L.R.Parra \& Giul. }\end{array}$ & ERV & $\mathrm{AM}, \mathrm{CE}, \mathrm{MA}$ & Almeida, T.E. et al. 3317 (BHCB) \\
\hline var. xeranthemoides & ERV & $\mathrm{AM}, \mathrm{CE}, \mathrm{MA}$ & C. A. Cid Ferreira et al. 5805 (US) \\
\hline Paepalanthus chiquitensis Herzog & SUB & $\mathrm{AM}$ & Lima, L.C.P. 640 (HUEFS) \\
\hline Paepalanthus fasciculatus (Rottb.) Kunth & ERV & $\mathrm{AM}, \mathrm{CE}$ & Carvalho, F.A. de 1906 (INPA) \\
\hline Paepalanthus guaraiensis Moldenke** & ERV & $\mathrm{CE}$ & Almeida, T.E. 3312 (HRCB) \\
\hline Paepalanthus polytrichoides Kunth & ERV & $\mathrm{AM}$ & Ferreira, C.A.C. 5500 (NY) \\
\hline Syngonanthus allenii var. brasiliensis Moldenke* & ERV & $\mathrm{AM}$ & Almeida, TE et al. 3316 (BHCB) \\
\hline Syngonanthus humboldtii (Kunth) Ruhland & ERV & $\mathrm{AM}, \mathrm{CE}$ & Lima, L.C.P. 646 (HUEFS) \\
\hline Syngonanthus setifolius Hensold & ERV & AM, CAA, CE, MA & $\begin{array}{l}\text { Almeida, C. \& Turma CB15_ME01 } 184 \\
\text { (INPA) } \\
\text { Almeida, C. \& Turma CB15_ME01 } 183 \\
\text { (INPA) }\end{array}$ \\
\hline Syngonanthus tenuis (Kunth) Ruhland & ERV & $\mathrm{AM}, \mathrm{CE}$ & Carvalho, F.A. de 2007 (INPA) \\
\hline \multicolumn{4}{|l|}{ Euphorbiaceae } \\
\hline Acalypha brasiliensis Mull.Arg. ** & $\mathrm{ARB}$ & AM, CAA, CE, MA & Junqueira, A.B. 218 (INPA) \\
\hline Acalypha cuneata Poepp & ARB & AM, MA & Almeida, C. \& Castro, A. 176 (INPA) \\
\hline Acalypha macrostachya Jacq.* & ARV & $\mathrm{AM}, \mathrm{CE}$ & Junqueira, A.B. 380 (EAFM) \\
\hline Alchornea discolor Poepp. & ARB & AM, CEE, MA & Almeida, C. \& Castro, A. 199 (INPA) \\
\hline Alchorneopsis floribunda (Benth.) Múll.Arg. & ARV & $\mathrm{AM}$ & Junqueira, A.B. 518 (INPA) \\
\hline Aparisthmium cordatum (A.Juss.) Baill. & $\mathrm{ARB}$ & AM, MA & $\begin{array}{l}\text { Almeida, C. \& Castro, A. } 211 \text { (INPA) } \\
\text { Junqueira, A.B. \& Neto, R.F. } 504\end{array}$ \\
\hline Conceveiba guianensis Aubl. & ARV & $\mathrm{AM}$ & (EAFM) \\
\hline Conceveiba martiana Baill. & ARV & $\mathrm{AM}$ & Mota, C.D.A. da 61463 (INPA) \\
\hline Croton glandulosus L. & $\mathrm{ARB}$ & $\begin{array}{l}\text { AM, CAA, CE, MA, } \\
\text { PAM, PAT }\end{array}$ & Almeida, C. \& Castro, A. 141 (INPA) \\
\hline Croton krukoffianus Croizat & $\mathrm{ARB}$ & $\mathrm{AM}, \mathrm{CE}$ & Ferreira, CID. 5813 (UB) \\
\hline Croton palanostigma Klotzsch. & ARV & AM & $\begin{array}{l}\text { Junqueira, A.B. \& Souza, S.A. } 626 \\
\text { (EAFM) }\end{array}$ \\
\hline Croton sampatik Mull.Arg. & $\mathrm{ARB}$ & $\mathrm{AM}$ & Ferreira, C.A.C. 5813 (INPA) \\
\hline Dendrothrix wurdackii Esser & ARB & $\mathrm{AM}$ & Ferreira, C.A.C. 5797 (INPA) \\
\hline Dodecastigma amazonicum Ducke & ARV & $\mathrm{AM}$ & Ferreira, C.A.C. 5613 (INPA) \\
\hline Hevea spruceana (Benth.) Mull.Arg. & ARV & $\begin{array}{c}\text { AM } \\
\text { AM, CAA, CE, MA, }\end{array}$ & Albernaz, A.L.K.M. 179 (INPA) \\
\hline Jatropha curcas L. \# & $\mathrm{ARB}$ & PAT & Mendes, R.F. et al. 24 (EAFM) \\
\hline Jatropha gossypifolia $\mathrm{L}$. & $\mathrm{ARB}$ & AM, CAA, MA & Almeida, C. \& Castro, A. 41 (INPA) \\
\hline Mabea angustifolia Spruce ex Benth. & ARV & $\mathrm{AM}$ & Almeida, C. \& Castro, A. 64 (INPA) \\
\hline Mabea longibracteata Esser & $\mathrm{ARB}$ & $\mathrm{AM}$ & Mota, C.D.A. da (INPA) \\
\hline Mabea nitida Spruce ex Benth. & ARV & $\mathrm{AM}, \mathrm{CE}$ & Viana, G.P. 98 (INPA) \\
\hline Mabea piriri Aubl. & ARV & AM, CE, MA & Mota, C.D.A. da (INPA) \\
\hline Mabea speciosa Müll. Arg. & ARB & AM & Almeida, C. \& Castro, A. 204 (INPA) \\
\hline
\end{tabular}




\begin{tabular}{|c|c|c|c|}
\hline Scientific name & Habit & $\begin{array}{l}\text { Phytogeographic } \\
\text { domain }\end{array}$ & Voucher \\
\hline Manihot esculenta Crantz & ARB & $\mathrm{AM}, \mathrm{CE}$ & Ferreira, C.A.C. 5822 (INPA) \\
\hline Manihot quinquepartita Huber ex D.J.Rogers \& Appan & $\mathrm{ARB}$ & $\mathrm{AM}, \mathrm{CAA}, \mathrm{CE}$ & Almeida, C. \& Castro, A. 251 (INPA) \\
\hline Maprounea guianensis Aubl. & $\mathrm{ARB}$ & $\mathrm{AM}, \mathrm{CE}, \mathrm{MA}$ & Silveira, A.L.P. 232 (UEC) \\
\hline Microstachys bidentata (Mart.\& Zucc.) Esser & LIA & AM, CAA, CE, MA & Ferreira, C.A.C. 5801 (NY) \\
\hline Microstachys corniculata (Vahl) Griseb. & SUB & AM, CAA, CE, MA & Almeida, C. \& Castro, A. 207 (INPA) \\
\hline Pausandra hirsuta Lanj. & $\mathrm{ARB}$ & $\mathrm{AM}$ & Ferreira, C.A.C. 5843 (INPA) \\
\hline Pausandra macropetala Ducke & ARV & $\mathrm{AM}$ & Ferreira, C.A.C. 5764 (INPA) \\
\hline Piranhea trifoliata Baill. & ARV & $\begin{array}{c}\text { AM } \\
\text { AM, CAA, CE, MT, }\end{array}$ & Viana, G.P. 78 (INPA) \\
\hline Ricinus communis L.\# & $\mathrm{ARB}$ & PAM, PAT & Almeida, C. \& Castro, A. 223 (INPA) \\
\hline Rhodothyrsus macrophyllus (Ducke) Esser & ARB & $\mathrm{AM}$ & Almeida, C. \& Castro, A. 247 (INPA) \\
\hline Sagotia racemosa Baill. & ARB & $\mathrm{AM}$ & Ferreira, C.A.C. 5759 (NY) \\
\hline Sandwithia guyanensis Lanj. & ARV & $\mathrm{AM}$ & Ferreira, C.A.C. 5759 (INPA) \\
\hline \multicolumn{4}{|l|}{ Fabaceae } \\
\hline $\begin{array}{l}\text { Abarema adenophora (Ducke) Barneby \& J.W.Grimes } \\
\text { Abarema campestris (Spruce ex Benth.) Barneby \& }\end{array}$ & ARB & $\mathrm{AM}$ & Almeida, C. \& Castro, A. 124 (INPA) \\
\hline J.W.Grimes & ARB & $\mathrm{AM}$ & Mota, C.D.A. da 61607 (INPA) \\
\hline Abarema laeta (Benth.) Barneby \& J.W.Grimes & $\mathrm{ARB}$ & $\mathrm{AM}$ & Mota, C.D.A. da (INPA) \\
\hline Alexa grandiflora Ducke & ARV & AM & $\begin{array}{l}\text { Junqueira, A.B. \& Souza, S.A. } 617 \\
\text { (INPA) } \\
\text { Junqueira, A.B. \& Souza, S.A. } 679\end{array}$ \\
\hline Amphiodon effusus Huber & ARV & AM, CAA, CE, MA & (INPA) \\
\hline Apuleia leiocarpa (Vogel) J.F.Macbr. & ARV & AM, CAA, CE, MA & Amoêdo, S.C. et al. 44 (EAFM) \\
\hline Bauhinia longicuspis Benth. & ARV & $\mathrm{AM}$ & Junqueira, A.B. 213 (INPA) \\
\hline Bauhinia rufa (Bong.) Steud.** & $\mathrm{ARB}$ & $\mathrm{CE}$ & Ferreira, C.A.C. 5818 (INPA) \\
\hline Cassia fastuosa Willd. ex Benth. & ARV & $\mathrm{AM}$ & Ferreira, C.A.C. 5505 (INPA) \\
\hline Cassia fastuosa Willd. ex Benth. var. fastuosa & ARV & AM & Ferreira, C.A.C. 5505 (NY) \\
\hline Cassia leiandra Benth. & ARV & $\begin{array}{c}\mathrm{AM} \\
\mathrm{AM}, \mathrm{CAA}, \mathrm{CE}\end{array}$ & Almeida, C. \& Castro, A. 101 (INPA) \\
\hline Centrosema vexillatum Benth. & TRE & $\begin{array}{c}\text { PAT } \\
\text { AM, CAA, CE, MA, }\end{array}$ & Ferreira, C.A.C. 5802 (INPA) \\
\hline $\begin{array}{l}\text { Chamaecrista desvauxii (Collad.) Killip } \\
\text { Chamaecrista langsdorffii (Kunth ex Vogel) Britton ex }\end{array}$ & $\mathrm{ARB}$ & PAT & Almeida, C. et al. 34 (INPA) \\
\hline Pittier & ERV & $\begin{array}{l}\text { AM, CAA, CE, MA } \\
\text { AM, CAA, CE, MA, }\end{array}$ & Lima, L.C.P. 652 (HUEFS) \\
\hline Chamaecrista flexuosa (L.) Greene & ERV & PAM, PAT & Giacomin, L.L. 1919 (BHCB) \\
\hline Clathrotropis nitida (Benth.) Harms & ARV & $\mathrm{AM}$ & Viana, G.P. 217 (INPA) \\
\hline Copaifera multijuga Hayne & ARV & AM & Viana, G.P. 219 (INPA) \\
\hline Crotalaria micans Link & $\mathrm{ARB}$ & $\begin{array}{l}\text { AM, CAA, CE, MA, } \\
\text { PAM, PAT }\end{array}$ & Almeida, C. \& Castro, A. 232 (INPA) \\
\hline Crudia amazonica Spruce ex Benth. & ARV & $\mathrm{AM}$ & Ferreira, C.A.C. 5777 (INPA) \\
\hline Dalbergia inundata Spruce ex Benth. & $\mathrm{ARB}$ & $\mathrm{AM}$ & Ferreira, C.A.C. 5543 (INPA) \\
\hline $\begin{array}{l}\text { Dalbergia riparia (Mart. ex Benth.) Benth. } \\
\text { Deguelia angulata (Ducke) A.M.G.Azevedo \& }\end{array}$ & LIA & $\mathrm{AM}$ & Viana, G.P. 90 (INPA) \\
\hline $\begin{array}{l}\text { R.A.Camargo } \\
\text { Deguelia rariflora (Mart. ex Benth.) G.P.Lewis \& Acev.- }\end{array}$ & LIA & $\mathrm{AM}$ & Krukoff, B.A. 7766 (NY) \\
\hline $\begin{array}{l}\text { Rodr. } \\
\text { Deguelia spruceana (Benth.) A.M.G.Azevedo \& }\end{array}$ & LIA & $\mathrm{AM}$ & Krukoff, B.A. 7760 (NY) \\
\hline R.A.Camargo & LIA & AM & Albernaz, A.L.K.M. 173 (INPA) \\
\hline Deguelia utilis (A.C.Sm.) A.M.G.Azevedo & TRE & $\mathrm{AM}$ & Krukoff, B.A. 7772 (NYBG) \\
\hline Desmodium adscendens (Sw.) DC\# & SUB & $\begin{array}{l}\text { AM, CAA, CE, MA, } \\
\text { PAM } \\
\text { AM, CAA, CE, MA, }\end{array}$ & Silva, G.P. 334 (HUEFS) \\
\hline Desmodium barbatum (L.) Benth. & SUB & $\begin{array}{l}\text { PAM, PAT } \\
\text { AM, CAA, CE, MA, }\end{array}$ & Lima, L.C.P. et al. 654 (UEC) \\
\hline Desmodium distortum (Aubl.) J.F.Macbr. & ARB & PAT & Lima, L.C.P. et al. 630 (UEC) \\
\hline Desmodium juruenense Hoehne & SUB & $\mathrm{AM}, \mathrm{CE}$ & Lima, L.C.P. 631 (HUEFS) \\
\hline
\end{tabular}


Table 1: continuation.

\begin{tabular}{|c|c|c|c|}
\hline Scientific name & Habit & $\begin{array}{c}\text { Phytogeographic } \\
\text { domain }\end{array}$ & Voucher \\
\hline Dialium guianense (Aubl.) Sandwith. & ARV & AM, MA & $\begin{array}{l}\text { Junqueira, A.B. \& Barros, R.N.S. } 391 \\
\text { (EAFM) }\end{array}$ \\
\hline Dinizia excelsa Ducke & ARV & $\mathrm{AM}$ & Amoêdo, S.C. et al. 43 (EAFM) \\
\hline Dioclea coriacea Benth & TRE & $\mathrm{AM}, \mathrm{CE}$ & Almeida, C. \& Castro, A. 125 (INPA) \\
\hline Dioclea scabra (Rich.) Maxwell & ARB & $\mathrm{AM}$ & $\begin{array}{l}\text { Ferreira, C.A.C. } 5826 \text { (INPA) } \\
\text { Junqueira, A.B. \& Neto, R.F. } 529\end{array}$ \\
\hline Diplotropis triloba Gleason. & ARV & $\mathrm{AM}$ & $(\mathrm{EAFM})$ \\
\hline Dipteryx magnifica (Ducke) Ducke & ARV & $\mathrm{AM}$ & Amoêdo, S.C. et al. 51 (EAFM) \\
\hline Dipteryx odorata (Aubl.) Willd. & ARV & $\mathrm{AM}$ & Viana, G.P. 96 (INPA) \\
\hline Dipteryx punctata (Blake) Amshoff. & ARV & $\mathrm{AM}$ & $\begin{array}{l}\text { Ferreira, C.A.C. } 5570 \text { (INPA) } \\
\text { Junqueira, A.B. \& Neto, R.F. } 440\end{array}$ \\
\hline Enterolobium schomburgkii (Benth.) Benth. & ARV & $\mathrm{AM}, \mathrm{CE}$ & (EAFM) \\
\hline Eperua oleifera Ducke & ARV & $\mathrm{AM}$ & Ferreira, C.A.C. 5791 (US) \\
\hline Eperua oleifera Ducke var. campestris Ducke & $\mathrm{ARB}$ & $\begin{array}{c}\text { AM } \\
\text { AM, CAA, CE, MA, }\end{array}$ & Ferreira, C.A.C. 5791 (INPA) \\
\hline Hymenaea courbaril $\mathrm{L}$. & ARV & PAT & Mendes, R.F. et al. 14 (EAFM) \\
\hline Hymenaea reticulata Ducke & ARV & $\mathrm{AM}$ & Amoêdo, S.C. et al. 45 (EAFM) \\
\hline Inga alba (Sw.) Willd. & ARV & $\mathrm{AM}, \mathrm{CE}$ & (EAFM) \\
\hline Inga cylindrica (Vell.) Mart. & $\mathrm{ARB}$ & $\mathrm{AM}, \mathrm{CE}, \mathrm{MA}$ & $\begin{array}{l}\text { Almeida, C. \& Castro, A. } 147 \text { (INPA) } \\
\text { Junqueira, A.B. \& Souza, S.A. } 588\end{array}$ \\
\hline Inga disticha Benth. & ARV & $\mathrm{AM}, \mathrm{CE}$ & (INPA) \\
\hline Inga edulis Mart. & ARB & AM, CAA, CE, MA & $\begin{array}{l}\text { Almeida, C. et al. } 35 \text { (INPA) } \\
\text { Junqueira A. \& \& Souz S A } 582\end{array}$ \\
\hline Inga grandiflora Ducke. & ARV & $\mathrm{AM}$ & $\begin{array}{l}\text { (EAFM) } \\
\text { Junqueira, A.B. \& Souza, S.A. } 690\end{array}$ \\
\hline Inga heterophylla Willd & ARV & $\mathrm{AM}, \mathrm{CE}$ & (EAFM) \\
\hline Inga ingoides (Rich.) Willd. & ARV & AM, CE, MA & $\begin{array}{l}\text { Junqueira, A.B. \& Neto, R.F. } 448 \\
\text { (EAFM) }\end{array}$ \\
\hline Inga lateriflora Miq. & ARV & $\mathrm{AM}, \mathrm{CE}$ & $\begin{array}{l}\text { Ferreira, C.A.C. } 5804 \text { (INPA) } \\
\end{array}$ \\
\hline Inga lomatophylla (Benth.) Pittier & ARV & $\mathrm{AM}$ & (EAFM) \\
\hline Inga longiflora Spruce ex Benth. & ARV & $\mathrm{AM}$ & $(\mathrm{EAFM})$ \\
\hline Inga macrophylla Kunth ex Willd. & ARV & $\mathrm{AM}$ & $\begin{array}{l}\text { Junqueira, A.B. } 389 \text { (INPA) } \\
\text { Junqueira, A.B. \& Souza, J.R. } 251\end{array}$ \\
\hline Inga marginata Willd & ARV & $\mathrm{AM}, \mathrm{CE}, \mathrm{MA}$ & $\begin{array}{l}\text { (EAFM) } \\
\text { Junqueira, A.B. \& Barros, R.N.S. } 326\end{array}$ \\
\hline Inga obidensis Ducke & ARV & $\mathrm{AM}$ & $($ EAFM) \\
\hline Inga panurensis Spruce ex Benth. & ARV & $\mathrm{AM}$ & Ferreira, C.A.C. 5804 (NY) \\
\hline Inga rubiginosa (Rich.) DC. & ARV & $\mathrm{AM}$ & $\begin{array}{l}\text { Almeida, C. \& Castro, A. } 67 \text { (INPA) } \\
\text { Junqueira, A.B. \& Neto, R.F. } 421\end{array}$ \\
\hline Inga umbellifera (Vahl) DC. & ARV & $\mathrm{AM}$ & (EAFM) \\
\hline Lecointea amazonica Ducke & ARV & $\mathrm{AM}$ & Viana, G.P. 110 (INPA) \\
\hline $\begin{array}{l}\text { Libidibia ferrea (Mart. ex Tul.) L.P.Queiroz. ** } \\
\text { Limadendron amazonica (Ducke) J.E. Meireles \& A.M.G. }\end{array}$ & $\mathrm{ARB}$ & CAA, CE, MA & Mendes, R.F. et al. 30 (EAFM) \\
\hline $\begin{array}{l}\text { Azevedo } \\
\text { Limadendron hostmannii (Benth.) Meireles \& }\end{array}$ & ARV & $\mathrm{AM}$ & Ferreira, C. A.C. 5829 (NY) \\
\hline A.M.G.Azevedo* & ARV & $\mathrm{AM}$ & $\begin{array}{l}\text { Ferreira, C.A.C. } 5829 \text { (INPA) } \\
\text { Junqueira, A.B. \& Barros, R.N.S. } 301\end{array}$ \\
\hline Machaerium floribundum Benth. & LIA & $\mathrm{AM}$ & $\begin{array}{l}\text { (EAFM) } \\
\text { Junqueira, A.B. \& Barros, R.N.S. } 385\end{array}$ \\
\hline Machaerium hoehneanum Ducke. & ARV & $\mathrm{AM}$ & $(\mathrm{EAFM})$ \\
\hline Machaerium leiophyllum (DC.) Benth. & LIA & $\mathrm{AM}$ & Viana, G.P. 59 (INPA) \\
\hline Macrolobium arenarium Ducke & $\mathrm{ARB}$ & $\mathrm{AM}$ & $\begin{array}{l}\text { Ferreira, C.A.C. } 5789 \text { (INPA) } \\
\text { Junqueira, A.B. \& Barros, R.N.S. } 359\end{array}$ \\
\hline Macrolobium limbatum Spruce ex Benth. & ARV & $\mathrm{AM}$ & $(\mathrm{EAFM})$ \\
\hline Macrolobium microcalyx Ducke & ARV & $\mathrm{AM}$ & Ferreira, C.A.C. 5558 (INPA) \\
\hline
\end{tabular}




\begin{tabular}{|c|c|c|c|}
\hline Scientific name & Habit & $\begin{array}{l}\text { Phytogeographic } \\
\text { domain }\end{array}$ & Voucher \\
\hline Mimosa guilandinae (DC.) Barneby & LIA & AM & Ferreira, C.A.C. 5526 (NY) \\
\hline Mimosa guilandinae var. spruceana (Benth.) Barneby & $\mathrm{ARB}$ & $\mathrm{AM}$ & Almeida, C. \& Castro, A. 129 (INPA) \\
\hline Mimosa invisa Mart. ex Colla & $\mathrm{ARB}$ & AM, CAA, CE, MA & Almeida, C. \& Castro, A. 198 (INPA) \\
\hline Mimosa myriadenia (Benth.) Benth. var. dispersa Barneby & ARB & $\mathrm{AM}$ & Ferreira, C.A.C. 5823 (INPA) \\
\hline Mimosa pudica $\mathrm{L}$. & SUB & AM, CAA, CE, MA & Almeida, C. et al. 5823 (INPA) \\
\hline Mimosa sensitiva $\mathrm{L}$. & ERV & AM, CAA, CE, MA & Almeida, C. et al. 57 (INPA) \\
\hline Ormosia macrocalyx Ducke & ARV & $\mathrm{AM}$ & $\begin{array}{l}\text { Almeida, C. } 83 \text { (INPA) } \\
\text { Junqueira, A.B. \& Souza, S.A. } 583\end{array}$ \\
\hline Ormosia grossa Rudd & ARV & AM & (EAFM) \\
\hline Pachyrhizus erosus (L.) Urb.\# & TRE & $\mathrm{AM}, \mathrm{CE}$ & Almeida, C. et al. 32 (INPA) \\
\hline Parkia discolor Spruce ex Benth. & ARV & $\mathrm{AM}$ & $\begin{array}{l}\text { Ferreira, C.A.C. } 5562 \text { (INPA) } \\
\text { Junqueira, A.B. \& Neto, R.F. } 539\end{array}$ \\
\hline Parkia nitida Miq. & ARV & $\mathrm{AM}$ & \\
\hline Parkia pendula (Willd.) Benth. ex Walp & ARV & AM, MA & (EAFM) \\
\hline Parkia ulei (Harms) Kuhlm. & ARV & $\mathrm{AM}$ & Ferreira, C.A.C. 5510 (INPA) \\
\hline $\begin{array}{l}\text { Peltogyne paniculata Benth. subsp. Paniculata } \\
\text { Peltogyne venosa (Vahl) Benth. ssp. densiflora (Spruce ex }\end{array}$ & $\mathrm{ARB}$ & AM & Ferreira, C.A.C. 5771 (US) \\
\hline Benth.) M.F.Silva & $\mathrm{ARB}$ & $\mathrm{AM}$ & Ferreira, C.A.C. 5771 (INPA) \\
\hline Piptadenia minutiflora Ducke & TRE & $\mathrm{AM}$ & Almeida, C. \& Castro, A. 114 (INPA) \\
\hline Plathymenia reticulata Benth.* & ARV & $\mathrm{AM}$ & Almeida, T.E. (HRCB) \\
\hline Pterocarpus amazonum (Benth.) Amshoff & ARV & $\mathrm{AM}$ & $\begin{array}{l}\text { Viana, G.P. } 88 \text { (INPA) } \\
\text { Junqueira, A.B. \& Souza, S.A. } 698\end{array}$ \\
\hline Pterocarpus rohrii Vahl. & ARV & AM, CAA, CE, MA & $(\mathrm{EAFM})$ \\
\hline Samanea saman (Jacq.) Merr. \#\# & ARV & AA & $\begin{array}{l}\text { Junqueira, A.B. } 240 \text { (INPA) } \\
\text { Junqueira, A.B. \& Souza, S.A. } 673\end{array}$ \\
\hline Schnella splendens (Kunth) Benth. & LIA & $\mathrm{AM}$ & (EAFM) \\
\hline Senna alata (L.) Roxb. & ARB & $\begin{array}{l}\text { AM, CAA, CE, MA, } \\
\text { PAT }\end{array}$ & Almeida, C. \& Castro, A. 38 (INPA) \\
\hline Senna cf macrophylla (Kunth) H.S.Irwin \& Barneby & LIA & $\mathrm{AM}$ & Almeida, C. \& Castro, A. 214 (INPA) \\
\hline Senna multijuga (Rich.) Irwin \& Barneby & ARV & $\mathrm{AM}, \mathrm{CAA}, \mathrm{CE}, \mathrm{MA}$ & Ferreira, C. A.C. 5505 (UB) \\
\hline Senna occidentalis (L.) Link & $\mathrm{ARB}$ & $\begin{array}{c}\text { AM, CAA, CE, MA, } \\
\text { PAT } \\
\text { AM, CAA, CE, MA, }\end{array}$ & Almeida, C. \& Castro, A. 569 (INPA) \\
\hline Senna silvestris (Vell.) H.S.Irwin \& Barneby & ARV & PAT & Junqueira, A.B. 311 (INPA) \\
\hline Senna tapajozensis (Ducke) H.S.Irwin \& Barneby & SUB & $\mathrm{AM}, \mathrm{CE}$ & Almeida, C. \& Castro, A. 51 (INPA) \\
\hline Senna undulata (Benth.) H.S.Irwin \& Barneby & $\mathrm{ARB}$ & $\mathrm{AM}$ & $\begin{array}{l}\text { Almeida, C. \& Castro, A. } 102 \text { (INPA) } \\
\text { Junqueira, A.B.; Barros, R.N.S. } 350\end{array}$ \\
\hline Stryphnodendron guianense (Aubl.) Benth. & ARV & AM, CAA & $(\mathrm{EAFM})$ \\
\hline Stryphnodendron pulcherrimum (Willd.) Hochr. & ARB & AM, MA & Almeida, C. \& Castro, A. 179 (INPA) \\
\hline Swartzia arborescens (Aubl.) Pittier & ARV & $\mathrm{AM}$ & $\begin{array}{l}\text { Ferreira, C.A.C. } 5838 \text { (INPA) } \\
\text { Junqueira, A.B. \& Neto, R.F. } 536\end{array}$ \\
\hline Swartzia cuspidata Spruce ex Benth & ARV & $\mathrm{AM}$ & (EAFM) \\
\hline Swartzia ingifolia Ducke & $\mathrm{ARB}$ & AM & Almeida, C. \& Castro, A. 146 (INPA) \\
\hline Swartzia laevicarpa Amshoff & ARV & $\mathrm{AM}$ & $\begin{array}{l}\text { Ferreira, C.A.C. } 5568 \text { (INPA) } \\
\text { Junqueira, A.B. \& Neto, R.F. } 551\end{array}$ \\
\hline Swartzia laurifolia Benth. & ARV & $\mathrm{AM}$ & $\begin{array}{l}\text { (EAFM) } \\
\text { Junqueira, A.B. \& Barros, R.N.S. } 315\end{array}$ \\
\hline Swartzia laxiflora Bong. ex Benth. & ARV & $\mathrm{AM}$ & $(\mathrm{EAFM})$ \\
\hline Swartzia lucida R.S.Cowan & ARV & $\mathrm{AM}$ & Krukoff, B.A. 7879 (NY) \\
\hline Swartzia oraria R.S.Cowan* & $\mathrm{ARB}$ & $\mathrm{AM}$ & $\begin{array}{l}\text { Almeida, C. \& Castro, A. } 149 \text { (INPA) } \\
\text { Junqueira, A.B. \& Barros, R.N.S. } 393\end{array}$ \\
\hline Swartzia polyphylla DC. & ARV & AM & $\begin{array}{l}\text { (EAFM) } \\
\text { Junqueira, A.B. \& Souza, S.A. } 641\end{array}$ \\
\hline Swartzia tessmannii Harms. & ARV & $\mathrm{AM}$ & $(\mathrm{EAFM})$ \\
\hline Tachigali candelabrum van der Werff & ARV & $\mathrm{AM}$ & Ferreira, C.A.C. 5780 (INPA) \\
\hline Tachigali eriopetala (Ducke) L.G.Silva \& H.C.Lima & ARV & $\mathrm{AM}$ & Ferreira, C.A.C. 5512 (INPA) \\
\hline
\end{tabular}


Table 1: continuation.

\begin{tabular}{|c|c|c|c|}
\hline Scientific name & Habit & $\begin{array}{l}\text { Phytogeographic } \\
\text { domain }\end{array}$ & Voucher \\
\hline Tachigali paniculata Aubl.. & ARV & $\mathrm{AM}$ & Silveira, A.L.P. 28 (UEC) \\
\hline Tachigali vulgaris L.G.Silva \& H.C.Lima & ARV & $\mathrm{AM}, \mathrm{CAA}, \mathrm{CE}$ & Silveira, A.L.P. 28 (RON) \\
\hline Tephrosia nitens Benth. & ARV & $\mathrm{AM}, \mathrm{CE}$ & Krukoff, B.A. 7889 (NY) \\
\hline Vatairea sericea (Ducke) Ducke & ARV & $\mathrm{AM}$ & Amoêdo, S.C. et al. 53 (EAFM) \\
\hline Vigna lasiocarpa (Mart. ex Benth.) Verdc. & ERV & $\begin{array}{l}\text { AM, CE, MA, PAT } \\
\text { AM, CAA, CE, MA, }\end{array}$ & Junqueira, A.B. 244 (INPA) \\
\hline Zornia latifolia Sm. & ERV & PAM, PAT & Glocimar P.S. 333 (CEN) \\
\hline Zygia racemosa (Ducke) Barneby \& J.W.Grimes. & ARV & $\mathrm{AM}$ & Amoêdo, S.C. et al. 56 (EAFM) \\
\hline \multicolumn{4}{|l|}{ Gentianaceae } \\
\hline Chelonanthus albus (Spruce ex Progel) V.M.Badillo & ERV & $\mathrm{AM}$ & Carvalho, F.A. de 2004 (INPA) \\
\hline Chelonanthus acutangulus (Ruiz \& Pav.) Gilg & ERV & $\mathrm{AM}$ & Almeida, C. et al. 58 (INPA) \\
\hline Schultesia brachyptera Cham. & ERV & AM, CAA, CE, MA & Ferreira, C.A.C. 5520 (INPA) \\
\hline Tachia grandiflora Maguire \& Weaver & ARV & $\mathrm{AM}$ & Almeida, C. \& Castro, A. 71 (INPA) \\
\hline Voyria tenella Hook. & ERV & $\mathrm{AM}$ & Ferreira, C.A.C. 5621 (INPA) \\
\hline Voyriella parviflora (Miq.) Miq. & ERV & $\mathrm{AM}, \mathrm{CE}, \mathrm{MA}$ & Ferreira, C.A.C. 5620 (INPA) \\
\hline \multicolumn{4}{|l|}{ Gesneriaceae } \\
\hline Codonanthopsis ulei Mansf. & SUB & $\mathrm{AM}$ & Almeida, T.E. 3625 (INPA) \\
\hline Drymonia coccinea (Aubl.) Wiehler & TRE & AM, MA & Almeida, C. \& Castro, A. 172 (INPA) \\
\hline Drymonia serrulata (Jacq.) Mart. & LIA & AM, CAA, CE, MA & Almeida, C. \& Castro, A. 244 (INPA) \\
\hline \multicolumn{4}{|l|}{ Goupiaceae } \\
\hline Goupia glabra Aubl. & ARV & $\mathrm{AM}, \mathrm{CE}$ & $\begin{array}{l}\text { Junqueira, A.B. \& Neto, R.F. } 423 \\
\text { (EAFM) }\end{array}$ \\
\hline \multicolumn{4}{|l|}{ Heliconiaceae } \\
\hline Heliconia acuminata L. C. Rich & ERV & $\mathrm{AM}$ & Almeida, C. et al. 20 (INPA) \\
\hline Heliconia chartacea Lane ex Barreiros & ERV & $\mathrm{AM}$ & Almeida, C. \& Castro, A. 115 (INPA) \\
\hline Heliconia densiflora Verl & ERV & AM & Almeida, C. \& Castro, A. 165 (INPA) \\
\hline Heliconia psittacorum L.f. & ERV & $\begin{array}{l}\text { AIM, CAA, CE, MAA, } \\
\text { PAT }\end{array}$ & Almeida, C. \& Castro, A. 76 (INPA) \\
\hline \multicolumn{4}{|l|}{ Humiriaceae } \\
\hline Humiria balsamifera (Aubl.) J.St.-Hil. & ARV & AM, CAA, CE, MA & Ferreira, C.A.C. 5810 (INPA) \\
\hline \multicolumn{4}{|l|}{ Hypericaceae } \\
\hline Vismia cayennensis (Jacq.) Pers. & ARB & $\mathrm{AM}, \mathrm{CE}$ & Almeida, C. \& Castro, A. 157 (INPA) \\
\hline Vismia guianensis (Aubl.) Choisy & ARB & AM, CAA, CE, MA & $\begin{array}{l}\text { Almeida, C. \& Castro, A. } 137 \text { (INPA) } \\
\text { Junqueira, A.B. \& Neto, R.F. } 498\end{array}$ \\
\hline Vismia gracilis Hieron. & ARV & $\mathrm{AM}, \mathrm{CE}$ & $\begin{array}{l}\text { (EAFM) } \\
\text { Junqueira, A.B. \& Souza, S.A. } 636\end{array}$ \\
\hline Vismia japurensis Reichardt. & ARV & $\mathrm{AM}, \mathrm{CE}$ & (EAFM) \\
\hline Vismia macrophylla Kunth & ARB & $\mathrm{AM}$ & $\begin{array}{l}\text { Almeida, C. \& Castro, A. } 218 \text { (INPA) } \\
\text { Junqueira, A.B. \& Neto, R.F. } 420\end{array}$ \\
\hline Vismia sandwithii Ewan. & ARV & $\mathrm{AM}, \mathrm{CE}$ & $(\mathrm{EAFM})$ \\
\hline \multicolumn{4}{|l|}{ Icacinaceae } \\
\hline Emmotum nitens (Benth.) Miers & ARV & $\begin{array}{l}\text { AM, CAA, CE, MA, } \\
\text { PAT }\end{array}$ & Silveira, A.L.P. 247 (RON) \\
\hline \multicolumn{4}{|l|}{ Ixonanthaceae } \\
\hline Ochthocosmus barrae Hallier f. & ARV & $\mathrm{AM}, \mathrm{CE}$ & Silveira, A.L.P. 248 (RON) \\
\hline \multicolumn{4}{|l|}{ Lacistemataceae } \\
\hline Lacistema aggregatum (Berg) Rusby. & ARB & $\mathrm{AM}, \mathrm{CE}$ & $\begin{array}{l}\text { Almeida, C. \& Turma CB15_ME01 } 186 \\
\text { (INPA) }\end{array}$ \\
\hline Lacistema polystachyum Schnizl. & ARB & $\mathrm{AM}$ & Silveira, A.L.P. $255(\mathrm{RON})$ \\
\hline \multicolumn{4}{|l|}{ Lamiaceae } \\
\hline Amasonia angustifolia Mart. \& Schauer ex Schauer & ARB & $\begin{array}{c}\text { AM, CE } \\
\text { AM, CE, MA, }\end{array}$ & Ferreira, C.A.C. 5579 (INPA) \\
\hline Hyptis brevipes Poit. & ARB & $\begin{array}{c}\text { PAM, PAT } \\
\text { AM, CAA, CE, MA, }\end{array}$ & Almeida, C. \& Castro, A. 194 (INPA) \\
\hline Leonotis nepetifolia (L.) R.Br. & ERV & PAT & Almeida, C. \& Castro, A. 245 (INPA) \\
\hline
\end{tabular}




\begin{tabular}{|c|c|c|c|}
\hline Scientific name & Habit & $\begin{array}{c}\text { Phytogeographic } \\
\text { domain }\end{array}$ & Voucher \\
\hline Ocimum campechianum Mill. & $\mathrm{ARB}$ & AM, CAA, CE, MA & Mendes, R.F. et al. 5 (EAFM) \\
\hline Scutellaria agrestis A.St.-Hil. ex Benth* & ERV & AM, MA & Mendes, R.F. et al. 56 (EAFM) \\
\hline Vitex triflora Vahl & ARV & $\mathrm{AM}, \mathrm{CAA}, \mathrm{CE}$ & Albuquerque, B.W.P. de 366 (INPA) \\
\hline \multicolumn{4}{|l|}{ Lauraceae } \\
\hline Endlicheria formosa A.C.Sm & ARV & $\mathrm{AM}$ & $\begin{array}{l}\text { Junqueira, A.B. \& Neto, R.F. } 515 \\
\text { (EAFM) }\end{array}$ \\
\hline Endlicheria glomerata $\mathrm{Mez}^{*}$ & ARV & $\mathrm{AM}, \mathrm{CE}, \mathrm{MA}$ & Ferreira, C.A.C. 5563 (MBM) \\
\hline Endlicheria macrophylla (Meisn.) Mez & ARV & $\mathrm{AM}$ & Ferreira, C.A.C. 5563 (INPA) \\
\hline Mezilaurus itauba (Meisn.) Taub. ex Mez. & ARB & $\mathrm{AM}$ & Almeida, C. et al. 15 (INPA) \\
\hline Nectandra cuspidata Nees & ARV & $\mathrm{AM}, \mathrm{CAA}, \mathrm{CE}$ & Krukoff, B.A. 6064 (US) \\
\hline Ocotea cernua (Nees) Mez & ARV & AM, CE, MA & $\begin{array}{l}\text { Albuquerque, B.W.P. de } 364 \text { (INPA) } \\
\text { Junqueira, A.B. \& Neto, R.F. } 528\end{array}$ \\
\hline Ocotea guianensis Aubl. & ARV & $\mathrm{AM}, \mathrm{CE}$ & \\
\hline Ocotea longifolia Kunth. & ARV & $\mathrm{AM}$ & (INPA) \\
\hline Ocotea oblonga (Meisn.) Mez & ARV & AM, MA & Junqueira, A.B. 471 (INPA) \\
\hline Ocotea splendens (Meisn.) Baill. & ARV & $\mathrm{AM}$ & $\begin{array}{l}\text { Junqueira, A.B. \& Neto, R.F. } 517 \\
\text { (EAFM) }\end{array}$ \\
\hline Persea americana Mill. **\# & ARV & MA & $\begin{array}{l}\text { Junqueira, A.B. \& Souza, S.A. } 622 \\
\text { (EAFM) }\end{array}$ \\
\hline \multicolumn{4}{|l|}{ Lecythidaceae } \\
\hline $\begin{array}{l}\text { Allantoma decandra (Ducke) S.A.Mori, Y.-Y.Huang \& } \\
\text { Prance }\end{array}$ & ARV & $\mathrm{AM}$ & Amoêdo, S.C. et al. 5 (EAFM) \\
\hline Bertholletia excelsa Bonpl. & ARV & $\mathrm{AM}$ & $\begin{array}{l}\text { Junqueira, A.B. \& Souza, S. A. } 676 \\
\text { (EAFM) }\end{array}$ \\
\hline Couratari stellata A.C.Sm. & ARV & $\mathrm{AM}$ & $\begin{array}{l}\text { Junqueira, A.B. \& Souza, S.A. } 646 \\
\text { (EAFM) }\end{array}$ \\
\hline Couratari tenuicarpa A.C.Sm. & ARV & $\mathrm{AM}$ & Ferreira, C.A.C. 5544 (INPA) \\
\hline Eschweilera atropetiolata S.A.Mori & ARV & $\mathrm{AM}$ & $\begin{array}{l}\text { Junqueira, A.B. \& Barros, R.N.S. } 370 \\
\text { (EAFM) } \\
\text { Junqueira, A.B. \& Souza, S.A. } 663\end{array}$ \\
\hline Eschweilera chartaceifolia S.A.Mori. & ARV & $\mathrm{AM}$ & (EAFM) \\
\hline Eschweilera coriacea (DC.) S.A.Mori & ARB & $\mathrm{AM}$ & Almeida, C. et al. 98 (INPA) \\
\hline Eschweilera micrantha (O.Berg) Miers & ARB & $\mathrm{AM}$ & Mota, C.D.A. da 61662 (INPA) \\
\hline Eschweilera ovalifolia (DC.) Nied. & ARV & $\mathrm{AM}$ & Viana, G.P. 65 (INPA) \\
\hline $\begin{array}{l}\text { Eschweilera truncata A.C.Sm. } \\
\text { Gustavia augusta L. }\end{array}$ & ARV & AM, MA & $\begin{array}{l}\text { Junqueira, A.B. \& Neto, R.F. } 514 \\
\text { (EAFM) } \\
\text { Junqueira, A.B. \& Souza, J.R. } 245 \\
\text { (EAFM) }\end{array}$ \\
\hline Gustavia hexapetala (Aubl.) Sm. & $\mathrm{ARB}$ & $\mathrm{AM}$ & Viana, G.P. 86 (INPA) \\
\hline Gustavia poeppigiana O.Berg & ARV & $\mathrm{AM}$ & Almeida, T.E. 3641 (INPA) \\
\hline \multicolumn{4}{|l|}{ Lentibulariaceae } \\
\hline Utricularia amethystina Salzm. ex A.St.-Hil. \& Girard & ERV & $\mathrm{AM}, \mathrm{CAA}, \mathrm{CE}, \mathrm{MA}$ & Ferreira, C.A.C. 5522 (INPA) \\
\hline Utricularia simulans Pilg. & ERV & AM, CAA, CE, MA & Lima, L.C.P. 650 (HUEFS) \\
\hline Utricularia triloba Benj. & ERV & AM, CAA, CE, MA & Lima, L.C.P. 637 (HUEFS) \\
\hline \multicolumn{4}{|l|}{ Loganiaceae } \\
\hline Antonia ovata Pohl. & ARV & $\mathrm{AM}, \mathrm{CE}, \mathrm{MA}$ & Silveira, A.L.P. 328 (UEC) \\
\hline Bonyunia antoniifolia Progel & $\mathrm{ARB}$ & $\mathrm{AM}, \mathrm{CE}$ & Giacomin, L.L. et al. 1915 (INPA) \\
\hline Bonyunia minor N.E.Br* & ARV & $\mathrm{AM}$ & Silveira, A.L.P. 15 (UEC) \\
\hline Strychnos hirsuta Spruce & ARB & $\mathrm{AM}$ & Mota, C.D.A. da 61573 (INPA) \\
\hline \multicolumn{4}{|l|}{ Loranthaceae } \\
\hline Passovia brasiliana Kuijt & ERV & AM, CAA, CE, MA & Ferreira, C.A.C. 5504 (INPA) \\
\hline Passovia pedunculata (Jacq.) Kuijt & ERV & $\mathrm{AM}, \mathrm{CE}$ & Ferreira, C.A.C. 5783 (INPA) \\
\hline Struthanthus marginatus (Desr.) Blume & ERV & AM, CAA, CE, MA & Almeida, C. \& Castro, A. 171 (INPA) \\
\hline
\end{tabular}


Table 1: continuation.

\begin{tabular}{|c|c|c|c|}
\hline Scientific name & Habit & $\begin{array}{c}\text { Phytogeographic } \\
\text { domain }\end{array}$ & Voucher \\
\hline \multicolumn{4}{|l|}{ Lythraceae } \\
\hline Cuphea antisyphilitica Kunth. & SUB & $\begin{array}{l}\text { AM, CAA, CE, MA, } \\
\text { PAM, PAT }\end{array}$ & Ferreira, C.A.C. 5523 (INPA) \\
\hline Cuphea sabulosa S.A.Graham & $\mathrm{ARB}$ & $\mathrm{AM}$ & Ferreira, C.A.C. 5811 (INPA) \\
\hline Physocalymma scaberrimum Pohl & ARV & $\mathrm{AM}, \mathrm{CE}$ & Viana, G.P. 216 (INPA) \\
\hline \multicolumn{4}{|l|}{ Malpighiaceae } \\
\hline Blepharandra heteropetala W.R.Anderson & $\mathrm{ARB}$ & $\mathrm{AM}$ & Ferreira, C.A.C. 5790 (NYBG) \\
\hline Byrsonima arthropoda A.Juss. & ARV & $\mathrm{AM}, \mathrm{CE}$ & Krukoff, B.A. 6035 (NY) \\
\hline Byrsonima chrysophylla Kunth & ARV & $\mathrm{AM}, \mathrm{CE}, \mathrm{MA}$ & Silveira, A.L.P. 41 (UEC) \\
\hline Byrsonima coccolobifolia Kunth & ARV & $\begin{array}{c}\text { AM, CE, MA } \\
\text { AM, CAA, CE, MA, }\end{array}$ & Silveira, A.L.P. 229 (UEC) \\
\hline Byrsonima crassifolia (L.) Kunth & ARB & PAT & $\begin{array}{l}\text { Almeida, C. } \text { et al. } 92 \text { (INPA) } \\
\text { Junqueira, A.B. \& Neto, R.F. } 573\end{array}$ \\
\hline Byrsonima crispa A.Juss. & ARV & AM, MA & (EAFM) \\
\hline Byrsonima japurensis A.Juss. & ARV & AM, MA & Viana, G.P. 102 (INPA) \\
\hline Byrsonima laxiflora Griseb.* & ARV & CE, MA, PAT & Silveira, A.L.P. 300 (RON) \\
\hline Byrsonima linguifera Cuatrec. & ARB & $\mathrm{AM}$ & Ferreira, C.A.C. 5503 (INPA) \\
\hline Byrsonima poeppigiana A.Juss. & ARV & $\mathrm{AM}$ & Silveira, A.L.P. 24 (RON) \\
\hline Byrsonima umbellata Mart. ex A.Juss.* & ARB & $\mathrm{AM}, \mathrm{CE}$ & Ferreira, C.A.C. 5800 (INPA) \\
\hline Excentradenia primaeva (W.R.Anderson) W.R.Anderson & TRE & $\mathrm{AM}$ & Almeida, C. \& Castro, A. 63 (INPA) \\
\hline Lophanthera longifolia (Kunth) Griseb. & ARB & AM & Almeida, C. \& Castro, A. 154 (INPA) \\
\hline Niedenzuella stannea (Griseb.) W.R.Anderson & LIA & AM, PAT & Ferreira, C.A.C. 5507 (INPA) \\
\hline Pterandra arborea Ducke & ARV & $\mathrm{AM}, \mathrm{CE}$ & Silveira, A.L.P. 24 (UEC) \\
\hline Stigmaphyllon sinuatum (DC.) A.Juss. & LIA & $\mathrm{AM}$ & Krukoff, B.A. 6038 (NY) \\
\hline \multicolumn{4}{|l|}{ Malvaceae } \\
\hline Apeiba membranacea Spruce ex Benth. & ARV & $\mathrm{AM}$ & $\begin{array}{l}\text { Junqueira, A.B. \& Neto, R.F. } 460 \\
\text { (EAFM) }\end{array}$ \\
\hline Apeiba tibourbou Aubl. & $\mathrm{ARB}$ & AM, CAA, CE, MA & $\begin{array}{l}\text { Almeida, C. \& Castro, A. } 87 \text { (INPA) } \\
\text { Junqueira, A.B. \& Barros, R.N.S. } 291\end{array}$ \\
\hline Ceiba pentandra (L.) Gaertn.* & ARV & $\mathrm{AM}$ & (EAFM) \\
\hline Eriotheca globosa (Aubl.) A.Robyns. & ARV & $\mathrm{AM}, \mathrm{CE}$ & $\begin{array}{l}\text { Junqueira, A.B. \& Barros, R.N.S. } 386 \\
\text { (EAFM) }\end{array}$ \\
\hline Eriotheca longitubulosa A.Robyns. & ARV & $\mathrm{AM}$ & $\begin{array}{l}\text { Junqueira, A.B. \& Neto, R.F. } 520 \\
\text { (EAFM) }\end{array}$ \\
\hline Gossypium barbadense L.\# & ARB & AM, CAA, MA & Almeida, C. \& Castro, A. 140 (INPA) \\
\hline Guazuma ulmifolia Lam. & ARV & AM, CAA, CE, MA & Mendes, R.F. et al. 57 (EAFM) \\
\hline Heliocarpus americanus L.** & ARV & DESC & Junqueira, A.B. 300 (INPA) \\
\hline Hibiscus furcellatus Lam. & ARB & $\mathrm{AM}, \mathrm{CE}, \mathrm{MA}$ & Lima, L.C.P. 641 (HUEFS) \\
\hline Hibiscus sabdariffa L. ***\# & ARB & DESC & Almeida, C. \& Castro, A. 97 (INPA) \\
\hline Huberodendron swietenioides (Gleason) Ducke & ARV & $\mathrm{AM}$ & $\begin{array}{l}\text { Lemos F.R. } 20541 \text { (NY) } \\
\text { Junqueira, A.B. \& Barros, R.N.S. } 328\end{array}$ \\
\hline Sterculia frondosa Rich & ARV & $\mathrm{AM}$ & $(\mathrm{EAFM})$ \\
\hline Pachira insignis (Sw.) Savigny & ARV & $\mathrm{AM}$ & Viana, G.P. 221 (INPA) \\
\hline Pachira nitida Kunth & ARV & $\mathrm{AM}$ & Ferreira, C.A.C. 5554 (INPA) \\
\hline Scleronema micranthum (Ducke) Ducke & ARV & AM & $\begin{array}{l}\text { Mota, C.D.A. da } 61658 \text { (INPA) } \\
\text { Junqueira, A.B. \& Souza, J.R. } 268\end{array}$ \\
\hline Theobroma cacao L. \# & ARV & AM, MA & $\begin{array}{l}\text { (EAFM) } \\
\text { Junqueira, A.B. \& Neto, R.F. } 400\end{array}$ \\
\hline Theobroma obovatum Klotzsch ex Benth. & ARV & $\mathrm{AM}$ & $\begin{array}{l}\text { (EAFM) } \\
\text { Junqueira, A.B. Barros, R.N.S. } 396\end{array}$ \\
\hline Theobroma speciosum Spreng. & ARV & $\mathrm{AM}$ & (EAFM) \\
\hline Vasivaea podocarpa Kuhlm. & ARV & $\mathrm{AM}$ & Albernaz, A.L.K.M. 183 (INPA) \\
\hline \multicolumn{4}{|l|}{ Marantaceae } \\
\hline $\begin{array}{l}\text { Goeppertia picturata (K.Koch \& Linden) Borchs. \& } \\
\text { S.Suárez }\end{array}$ & ERV & $\mathrm{AM}, \mathrm{CE}, \mathrm{MA}$ & Viana, G.P. (INPA) \\
\hline Ischnosiphon obliquus (Rudge) Körn. & ERV & $\mathrm{AM}$ & Almeida, C. \& Castro, A. 202 (INPA) \\
\hline
\end{tabular}




\begin{tabular}{|c|c|c|c|}
\hline Scientific name & Habit & $\begin{array}{c}\text { Phytogeographic } \\
\text { domain }\end{array}$ & Voucher \\
\hline Monotagma densiflorum (Körn.) K.Schum. & ERV & $\mathrm{AM}, \mathrm{CE}$ & Almeida, C. \& Castro, A. 170 (INPA) \\
\hline Monotagma floribundum Hagberg \& R. Erikss. & $\mathrm{ARB}$ & $\mathrm{AM}$ & Mota, C.D.A. da 61578 (INPA) \\
\hline \multicolumn{4}{|l|}{ Marcgraviaceae } \\
\hline Souroubea guianensis Aubl. & LIA & AM, MA & Viana, G.P. 101 (INPA) \\
\hline \multicolumn{4}{|l|}{ Melastomataceae } \\
\hline Adelobotrys microcarpus Schulman & SUB & $\mathrm{AM}$ & Almeida, C. et al. 16 (INPA) \\
\hline Bellucia acutata Pilger & ARV & $\mathrm{AM}, \mathrm{CE}$ & Junqueira, A.B. 580 (INPA) \\
\hline Bellucia dichotoma Cogn & $\mathrm{ARB}$ & $\mathrm{AM}$ & Almeida, C. \& Castro, A. 49 (INPA) \\
\hline Bellucia grossularioides (L.) Triana. & ARV & $\mathrm{AM}, \mathrm{CE}$ & Lombardi, J.A. et al. 10105 (UNOP) \\
\hline Clidemia capitellata (Bonpl.) D.Don & SUB & AM, CAA, CE, MA & Almeida, C. et al. 47 (INPA) \\
\hline Clidemia hirta (L.) D.Don & ARV & AM, CAA, CE, MA & Krukoff, B.A. 6012 (NY) \\
\hline Clidemia rubra (Aubl.) Mart. & $\mathrm{ARB}$ & $\mathrm{AM}, \mathrm{CE}$ & Almeida, C. \& Castro, A. 229 (INPA) \\
\hline Macairea multinervia Benth. & $\mathrm{ARB}$ & $\mathrm{AM}$ & Ferreira, C.A.C. 5516 (INPA) \\
\hline Macairea thyrsiflora DC. & ARB & $\mathrm{AM}, \mathrm{CE}$ & Mota, C.D.A. da 61553 (INPA) \\
\hline Miconia affinis DC. & ARV & AM, CE, MA & $\begin{array}{l}\text { Junqueira, A.B.\& Souza, J.R. } 222 \\
\text { (EAFM) }\end{array}$ \\
\hline Miconia ciliata (Rich.) DC. & ARB & $\mathrm{AM}, \mathrm{CAA}, \mathrm{CE}, \mathrm{MA}$ & Silveira, A.L.P. 324 (UEC) \\
\hline Miconia cuspidata Naudin & ARV & $\mathrm{AM}, \mathrm{CE}, \mathrm{MA}$ & $\begin{array}{l}\text { Junqueira, A.B. \& Neto, R.F. } 495 \\
\text { (EAFM) } \\
\text { Junqueira A.B \& Neto R.F. } 503\end{array}$ \\
\hline Miconia dolichorrhyncha Naudin & ARV & $\mathrm{AM}, \mathrm{CE}$ & $\begin{array}{l}\text { Junqueira, A.B. \& Neto, K.F. } 503 \\
\text { (EAFM) }\end{array}$ \\
\hline Miconia dispar Benth. & $\mathrm{ARB}$ & $\mathrm{AM}$ & Almeida, C. \& Castro, A. 77 (INPA) \\
\hline Miconia fallax DC. & $\mathrm{ARB}$ & $\mathrm{AM}$ & Silveira, A.L.P. 32 (UEC) \\
\hline Miconia ibaguensis (Bonpl.) Triana. & $\mathrm{ARB}$ & AM, CAA, CE, MA & Silveira, A.L.P. 36 (UEC) \\
\hline Miconia minutiflora (Bonpl.) DC. & ARV & AM, CAA, CE, MA & Junqueira, A.B. 211 (INPA) \\
\hline Miconia nervosa (Sm.) Triana & $\mathrm{ARB}$ & AM, CAA, CE, MA & Ferreira, C.A.C. 5532 (INPA) \\
\hline Miconia poeppigii Triana & ARV & $\mathrm{AM}$ & Almeida, C.; Castro, A. 120 (INPA) \\
\hline Miconia prasina (Sw.) DC. & ARV & AM, CAA, CE, MA & Junqueira, A.B. 521 (INPA) \\
\hline Miconia rhytidophylla Naudin & $\mathrm{ARB}$ & $\mathrm{AM}$ & Mota, C.D.A. da 61669 (INPA) \\
\hline Miconia rubiginosa (Bonpl.) DC. & ARB & $\mathrm{AM}, \mathrm{CE}$ & Silveira, A.L.P. 305 (RON) \\
\hline Miconia stenostachya DC. & $\mathrm{ARB}$ & AM, CAA, CE, MA & Almeida, C. \& Castro, A. 180 (INPA) \\
\hline Miconia tillettii Wurdack & ARV & $\mathrm{AM}$ & Junqueira, A.B. 558 (INPA) \\
\hline Miconia tomentosa (Rich.) D.Don & ARB & $\mathrm{AM}, \mathrm{CE}, \mathrm{MA}$ & Almeida, C.; Castro, A. 153 (INPA) \\
\hline Mouriri acutiflora Naudin & ARB & $\mathrm{AM}, \mathrm{CE}$ & Albernaz, A.L.K.M. 169 (INPA) \\
\hline Siphanthera subtilis Pohl ex DC. & ERV & $\mathrm{AM}, \mathrm{CE}$ & Ferreira, C.A.C. 5793 (US) \\
\hline Tococa bullifera DC. & ARB & $\mathrm{AM}$ & Almeida, C. \& Castro, A. 230 (INPA) \\
\hline Tococa guianensis Aubl. & $\mathrm{ARB}$ & AM, CE, PAT & Almeida, C. \& Castro, A. 134 (INPA) \\
\hline \multicolumn{4}{|l|}{ Meliaceae } \\
\hline $\begin{array}{l}\text { Cedrela odorata L. } \\
\text { Guarea humaitensis T.D.Penn }\end{array}$ & ARV & $\begin{array}{c}\text { AM, CAA, CE, MA } \\
\text { AM }\end{array}$ & $\begin{array}{l}\text { Viana, G.P. } 105 \text { (INPA) } \\
\text { Junqueira, A.B. \& Souza, J.R. } 283 \\
\text { (EAFM) }\end{array}$ \\
\hline Guarea cf kunthiana A.Juss. & $\mathrm{ARB}$ & AM, CE, MA & Almeida, C. \& Castro, A. 228 (INPA) \\
\hline Guarea silvatica C.DC. & ARV & $\mathrm{AM}$ & Ferreira, C.A.C. 5612 (INPA) \\
\hline Trichilia fasciculata T.D.Penn. & ARV & $\mathrm{AM}$ & Ferreira, C.A.C. 5529 (INPA) \\
\hline $\begin{array}{l}\text { Trichilia micrantha Benth. } \\
\text { Trichilia rubra C.DC. }\end{array}$ & ARV & $\begin{array}{l}\mathrm{AM}, \mathrm{CE} \\
\text { AM }\end{array}$ & $\begin{array}{l}\text { Ferreira, C.A.C. } 5555 \text { (INPA) } \\
\text { Junqueira, A.B. \& Neto, R.F. } 469 \\
\text { (EAFM) }\end{array}$ \\
\hline \multicolumn{4}{|l|}{ Menispermaceae } \\
\hline Abuta grandifolia (Mart.) Sandwith & $\mathrm{ARB}$ & $\begin{array}{l}\text { AM, CAA, CE, } \\
\text { PAT }\end{array}$ & Ferreira, C.A.C. 5534 (INPA) \\
\hline \multicolumn{4}{|l|}{ Moraceae } \\
\hline Brosimum acutifolium Huber & ARV & AM, PAT & $\begin{array}{l}\text { Junqueira, A.B. \& Souza, S.A. } 693 \\
\text { (EAFM) }\end{array}$ \\
\hline Brosimum acutifolium subsp. interjectum C.C.Berg & ARV & $\mathrm{AM}$ & Moreira, L.P. (INPA) \\
\hline
\end{tabular}


Table 1: continuation.

\begin{tabular}{|c|c|c|c|}
\hline Scientific name & Habit & $\begin{array}{l}\text { Phytogeographic } \\
\text { domain }\end{array}$ & Voucher \\
\hline Brosimum guianense (Aubl.) Huber & ARV & AM, CAA, CE, MA & $\begin{array}{l}\text { Junqueira, A.B. \& Souza, S.A. } 644 \\
\text { (EAFM) }\end{array}$ \\
\hline Brosimum lactescens (S.Moore) C.C.Berg. & ARV & AM, CE, MA & $\begin{array}{l}\text { Junqueira, A.B.\& Barros, R.N.S. } 318 \\
\text { (EAFM) }\end{array}$ \\
\hline Brosimum rubescens Taub. & ARV & $\mathrm{AM}, \mathrm{CE}, \mathrm{MA}$ & Amoêdo, S.C. et al. 52 (EAFM) \\
\hline Castilla ulei Warb. & ARV & $\mathrm{AM}$ & $\begin{array}{l}\text { Junqueira, A.B. \& Barros, R.N.S. } 333 \\
\text { (EAFM) }\end{array}$ \\
\hline Clarisia biflora Ruiz \& Pav. & ARV & $\mathrm{AM}$ & $\begin{array}{l}\text { Junqueira, A.B. \& Souza, J.R. } 278 \\
\text { (EAFM) }\end{array}$ \\
\hline Clarisia ilicifolia (Spreng.) Lanj. \& Rossberg. & ARV & AM, MA & $\begin{array}{l}\text { Junqueira, A.B. \& Barros, R.N.S. } 322 \\
\text { (EAFM) }\end{array}$ \\
\hline Clarisia racemosa Ruiz \& Pav. & ARV & AM, MA & Amoêdo, S.C. et al. 36 (EAFM) \\
\hline Ficus amazonica (Miq.) Miq. & ARV & $\mathrm{AM}$ & Albernaz, A.L.K.M. 170 (INPA) \\
\hline Ficus eximia Schott & ARV & AM, CE, MA & Viana, G.P. 63 (INPA) \\
\hline Ficus maxima Mill. & ARV & $\mathrm{AM}$ & $\begin{array}{l}\text { Junqueira, A.B. \& Souza, J.R. } 273 \\
\text { (EAFM) }\end{array}$ \\
\hline Ficus obtusiuscula (Miq.) Miq. & ARB & AM, CAA, CE, MA & Ferreira, C.A.C. 5774 (INPA) \\
\hline Helianthostylis sprucei Baill. & ARV & $\mathrm{AM}$ & (EAFM) \\
\hline Maclura tinctoria $($ L.) D. Don ex Steud. & ARV & $\begin{array}{l}\text { AM, CAA, CE, MA, } \\
\text { PAM, PAT }\end{array}$ & $\begin{array}{l}\text { Junqueira, A.B. \& Barros, R.N.S. } 287 \\
\text { (EAFM) }\end{array}$ \\
\hline Maquira calophylla (Poepp. \& Endl.) C.C.Berg & ARV & $\mathrm{AM}, \mathrm{CE}$ & $\begin{array}{l}\text { Junqueira, A.B. \& Barros, R.N.S. } 358 \\
\text { (EAFM) }\end{array}$ \\
\hline Maquira coriacea (H.Karst.) C.C.Berg & ARV & $\mathrm{AM}, \mathrm{CE}$ & Viana, G.P. 100 (INPA) \\
\hline Maquira sclerophylla (Ducke) C.C.Berg & ARV & $\mathrm{AM}$ & $\begin{array}{l}\text { Junqueira, A.B. \& Barros, R.N.S. } 325 \\
\text { (EAFM) }\end{array}$ \\
\hline Naucleopsis stipularis Ducke & ARV & $\mathrm{AM}$ & Ferreira, C.A.C. 5584 (INPA) \\
\hline Perebea guianensis Aubl. & ARV & $\mathrm{AM}, \mathrm{CAA}, \mathrm{CE}$ & $\begin{array}{l}\text { Mota, C.D.A. da } 61602 \text { (INPA) } \\
\text { Junqueira, A.B. \& Neto, R.F. } 451\end{array}$ \\
\hline Perebea mollis (Poepp. \& Endl.) Huber. & ARV & $\mathrm{AM}, \mathrm{CAA}, \mathrm{CE}$ & $\begin{array}{l}\text { (EAFM) } \\
\text { Junqueira A.B \& Souza J.R. } 257\end{array}$ \\
\hline Pseudolmedia laevis (Ruiz \& Pav.) J.F.Macbr. & ARV & $\mathrm{AM}$ & (EAFM) \\
\hline Sorocea muriculata Miq. & ARV & AM, PAT & Almeida, T.E. 3618 (INPA) \\
\hline Sorocea muriculata Miq. subsp. muriculata & ARV & AM, PAT & Almeida, T.E. 3623 (INPA) \\
\hline Sorocea pubivena Hemsl. & ARV & $\mathrm{AM}$ & $\begin{array}{l}\text { Junqueira, A.B. } 256 \text { (INPA) } \\
\text { Junqueira, A.B. \& Neto, R.F. } 508\end{array}$ \\
\hline Sorocea muriculata subsp. uaupensis (Baill.) C.C.Berg & ARV & $\mathrm{AM}$ & $(\mathrm{EAFM})$ \\
\hline Trymatococcus amazonicus Poepp. \& Endl. & $\mathrm{ARB}$ & $\mathrm{AM}$ & Viana, G.P. 85 (INPA) \\
\hline \multicolumn{4}{|l|}{ Muntingiaceae } \\
\hline Muntingia calabura $\mathrm{L}$. & ARB & $\mathrm{AM}$ & Krukoff, B.A. 6016 (US) \\
\hline \multicolumn{4}{|l|}{ Myristicaceae } \\
\hline Iryanthera lancifolia Ducke & ARV & $\mathrm{AM}$ & Ferreira, C.A.C. 5588 (UPCB) \\
\hline Iryanthera macrophylla (Benth.) Warb. & ARV & $\mathrm{AM}$ & Mota, C.D.A. da 61603 (INPA) \\
\hline Iryanthera tessmannii Markgr. & ARV & $\mathrm{AM}$ & Ferreira, C.A.C. 5614 (NY) \\
\hline Iryanthera tricornis Ducke & ARV & $\mathrm{AM}$ & Mota, C.D.A. da 61663 (INPA) \\
\hline Iryanthera ulei Warb. & ARV & $\mathrm{AM}$ & Ferreira, C.A.C. 58 (EAFM) \\
\hline Osteophloeum platyspermum (Spruce ex A.DC.) Warb. & ARV & $\mathrm{AM}$ & Amoêdo, S.C. et al. 58 (EAFM) \\
\hline Virola calophylla Warb. & ARV & $\mathrm{AM}$ & Viana, G.P. 77 (INPA) \\
\hline Virola elongata (Benth.) Warb. & ARV & $\mathrm{AM}$ & Ferreira, C.A.C. 5557 (INPA) \\
\hline Virola obovata Ducke & ARV & $\mathrm{AM}$ & Albernaz, A.L.K.M. 172 (INPA) \\
\hline Virola pavonis (A.DC.) A.C.Sm. & ARV & $\mathrm{AM}$ & Mota, C.D.A. da 61619 (INPA) \\
\hline Virola sebifera Aubl. & ARV & AM, CE, MA & Lombardi, J.A. 60664 (HECB) \\
\hline Virola surinamensis (Rol. ex Rottb.) Warb. & ARV & $\mathrm{AM}, \mathrm{CAA}$ & Albernaz, A.L.K.M. 181 (INPA) \\
\hline \multicolumn{4}{|l|}{ Myrsinaceae } \\
\hline $\begin{array}{l}\text { Cybianthus fulvopulverulentus subsp. magnoliifolius (Mez) } \\
\text { Pipoly }\end{array}$ & $\mathrm{ARB}$ & $\mathrm{AM}$ & Mota, C.D.A. da 61552 (INPA) \\
\hline
\end{tabular}




\begin{tabular}{|c|c|c|c|}
\hline Scientific name & Habit & $\begin{array}{l}\text { Phytogeographic } \\
\text { domain }\end{array}$ & Voucher \\
\hline \multicolumn{4}{|l|}{ Myrtaceae } \\
\hline Eugenia caducibracteata Mazine & ARV & AM & Junqueira, A.B. 527 (INPA) \\
\hline Eugenia citrifolia Poir & ARV & $\mathrm{AM}$ & Junqueira, A.B. \& Neto, R.F. 531 EAFM) \\
\hline Eugenia cf. cuspidifolia DC. & $\mathrm{ARB}$ & $\mathrm{AM}$ & Almeida, C. \& Castro, A. 216 (INPA) \\
\hline Eugenia ferreiraeana O.Berg & $\mathrm{ARB}$ & $\mathrm{AM}$ & Ferreira, C.A.C. 5537 (INPA) \\
\hline Eugenia gomesiana O.Berg & ARV & $\begin{array}{c}\text { AM } \\
\text { AM, CAA, CE, MA. }\end{array}$ & Viana, G.P. 97 (INPA) \\
\hline $\begin{array}{l}\text { Eugenia lambertiana DC. } \\
\text { Eugenia omissa McVaugh }\end{array}$ & $\mathrm{ARB}$ & PAT & $\begin{array}{l}\text { Ferreira, C.A.C. } 5775 \text { (INPA) } \\
\text { Junqueira, A.B. \& Neto, R.F. } 427 \\
\text { (EAFM) }\end{array}$ \\
\hline Eugenia patens Poir. & ARV & $\mathrm{AM}$ & $\begin{array}{l}\text { Krukoff, B. A. } 6021 \text { (US) } \\
\text { Junqueira, A.B.; Souza, S.A. } 692\end{array}$ \\
\hline Myrcia aliena McVaugh. & ARV & AM & $(\mathrm{EAFM})$ \\
\hline Myrcia bracteata (Rich.) DC. & ARV & $\mathrm{AM}, \mathrm{CE}$ & Albuquerque, B.W.P. de 365 (INPA) \\
\hline Myrcia gigas $\mathrm{McVaugh}$ & ARV & $\mathrm{AM}$ & Junqueira, A.B.; Souza, J.R. 276 (INPA) \\
\hline Myrcia guianensis (Aubl.) DC. & ARB & AM, CAA, CE, MA & Almeida, C. \& Castro, A. 196 (INPA) \\
\hline Myrcia inaequiloba (DC.) Lemée & ARV & AM, MA & Krukoff, B.A. 5997 (SP) \\
\hline Myrcia multiflora (Lam.) DC. & ARV & $\mathrm{AM}, \mathrm{CAA}, \mathrm{CE}, \mathrm{MA}$ & $\begin{array}{l}\text { Almeida, C. \& Castro, A. } 195 \text { (INPA) } \\
\text { Junqueira, A.B. \& Souza, J.R. } 247 \\
\text { (EAFM) }\end{array}$ \\
\hline Myrcia splendens (Sw.) DC. & ARV & $\begin{array}{c}\text { AM, CAA, CE, MA, } \\
\text { PAT }\end{array}$ & Silveira, A.L.P. 318 (UEC) \\
\hline Myrcia sylvatica (G.Mey.) DC. & ARV & $\mathrm{AM}, \mathrm{CAA}, \mathrm{CE}$ & Junqueira, A.B. 507 (INPA) \\
\hline Myrcia umbraticola (Kunth) E.Lucas \& C.E.Wilson & $\mathrm{ARB}$ & $\mathrm{AM}$ & $\begin{array}{l}\text { Krukoff, B.A. } 5997 \text { (NY) } \\
\text { Junqueira, A.B. \& Souza, S.A. } 586\end{array}$ \\
\hline Psidium guineense $\mathrm{Sw}$. & ARV & AM, CAA, CE, MA & $(\mathrm{EAFM})$ \\
\hline Syzygium cumini (L.) Skeels.\# & ARV & AM, CE, MA, PAT & Krukoff, B.A. 6014 (SP) \\
\hline \multicolumn{4}{|l|}{ Nyctaginaceae } \\
\hline $\begin{array}{l}\text { Guapira opposita (Vell.) Reitz } \\
\text { Neea filipes Huber. }\end{array}$ & ARV & $\begin{array}{l}\text { AM, CAA, CE, MA } \\
\text { DESC }\end{array}$ & $\begin{array}{l}\text { Junqueira, A.B. \& Souza, J.R. } 250 \\
\text { (EAFM) } \\
\text { Junqueira, A.B. \& Souza, J.R. } 281 \\
\text { (EAFM) }\end{array}$ \\
\hline Neea madeirana Standl. & ARV & $\mathrm{AM}$ & Mota, C.D.A. da 61460 (INPA) \\
\hline Neea oppositifolia Ruiz \& Pav. & ARV & $\mathrm{AM}, \mathrm{CE}$ & Krukoff, B.A. 6058 (NYBG) \\
\hline Neea virens Poepp. ex Heimerl & ARB & DESC & Viana, G.P. 67 (INPA) \\
\hline \multicolumn{4}{|l|}{ Nymphaeceae } \\
\hline Nymphaea glandulifera Rodschied & ERV & $\mathrm{AM}, \mathrm{CE}$ & Almeida, C. \& Bacuri, J. 48 (INPA) \\
\hline \multicolumn{4}{|l|}{ Ochnaceae } \\
\hline Ouratea castaneifolia (DC.) Engl. & ARV & AM, CAA, CE, MA & $\begin{array}{l}\text { Junqueira, A.B. \& Souza, J.R. } 260 \\
\text { (EAFM) }\end{array}$ \\
\hline Ouratea spectabilis (Mart.) Engl.* & ARV & $\mathrm{CE}$ & Silveira, A.L.P. 241 (RON) \\
\hline Sauvagesia longifolia Eichler & ARV & AM, MA & Giacomin, L.L. et al. 1928 (BHCB) \\
\hline Sauvagesia racemosa A.St.-Hil. & $\mathrm{ARB}$ & AM, CAA, CE, MA & Ferreira, C.A.C. 5796 (INPA) \\
\hline Wallacea insignis Spruce ex Benth. \& Hook.f. & ARV & $\mathrm{AM}$ & Ferreira, C.A.C. 5536 (INPA) \\
\hline \multicolumn{4}{|l|}{ Olacaceae } \\
\hline $\begin{array}{l}\text { Aptandra tubicina (Poepp.) Benth. ex Miers. } \\
\text { Heisteria densifrons Engl. }\end{array}$ & ARV & AM, MA & $\begin{array}{l}\text { Junqueira, A.B. \& Barros, R.N.S. } 299 \\
\text { (EAFM) } \\
\text { Junqueira, A.B. \& Neto, R.F. } 398 \\
\text { (EAFM) }\end{array}$ \\
\hline \multicolumn{4}{|l|}{ Onagraceae } \\
\hline Ludwigia hyssopifolia (G.Don) Exell & $\mathrm{ARB}$ & $\begin{array}{c}\text { AM, MA, PAT } \\
\text { AM, CAA, CE, MA, }\end{array}$ & Almeida, C. \& Castro, A. 192 (INPA) \\
\hline Ludwigia octovalvis (Jacq.) P.H. Raven. & SUB & PAT & Lima, L.C.P. 643 (CGMS) \\
\hline \multicolumn{4}{|l|}{ Orchidaceae } \\
\hline Coryanthes macrantha (Hook.) Hook. & ERV & $\mathrm{AM}$ & Garcia, K. (INPA) \\
\hline Habenaria glazioviana Kraenzl. & ERV & AM, CAA, CE & Ferreira, C.A.C. 5575 (INPA) \\
\hline Ionopsis satyrioides (Sw.) Lindl. & ERV & $\mathrm{AM}$ & Krukoff, B.A. 6062 (NY) \\
\hline
\end{tabular}


Table 1: continuation.

\begin{tabular}{|c|c|c|c|}
\hline Scientific name & Habit & $\begin{array}{c}\text { Phytogeographic } \\
\text { domain }\end{array}$ & Voucher \\
\hline Liparis nervosa (Thumb.) Lindl. & ERV & AM, CAA, CE, MA & Ferreira, C.A.C. 5574 (INPA) \\
\hline \multicolumn{4}{|l|}{ Passifloraceae } \\
\hline Passiflora coccinea Aubl. & LIA & $\mathrm{AM}, \mathrm{CAA}$ & Almeida, C. et al. 21 (INPA) \\
\hline Passiflora glandulosa Cav. & TRE & $\begin{array}{c}\mathrm{AM} \\
\mathrm{AM}, \mathrm{CAA}, \mathrm{CE}, \mathrm{MA},\end{array}$ & Almeida, C. \& Castro, A. 107 (INPA) \\
\hline Passiflora misera Kunth & TRE & PAT & Almeida, C. \& Castro, A. 142 (INPA) \\
\hline Passiflora nitida Kunth & TRE & AM, CAA, CE & Almeida, C. \& Castro, A. 233 (INPA) \\
\hline \multicolumn{4}{|l|}{ Pentaphylacaceae } \\
\hline Ternstroemia cf krukoffiana Kobuski & ARV & $\mathrm{AM}$ & Almeida, C. et al. 91 (INPA) \\
\hline \multicolumn{4}{|l|}{ Peraceae } \\
\hline $\begin{array}{l}\text { Chaetocarpus echinocarpus (Baill.) Ducke. } \\
\text { Chaetocarpus schomburgkianus (Kuntze) Pax \& K.Hoffm. }\end{array}$ & ARV & $\mathrm{AM}, \mathrm{CAA}, \mathrm{CE}$ & $\begin{array}{l}\text { Lombardi } 10090 \text { (HRBC) } \\
\text { Junqueira, A.B. \& Neto, R.F. } 544 \\
\text { (EAFM) }\end{array}$ \\
\hline Pera bicolor (Klotzsch) Múll.Arg. & ARV & $\mathrm{AM}$ & Ferreira, C.A.C. 5564 (INPA) \\
\hline Pera decipiens (Müll.Arg.) Müll.Arg & $\mathrm{ARB}$ & AM & Silveira, A.L.P. 16 (UEC) \\
\hline \multicolumn{4}{|l|}{ Phyllanthaceae } \\
\hline Phyllanthus stipulatus (Raf.) G.L.Webster & SUB & $\mathrm{AM}, \mathrm{CE}, \mathrm{MA}$ & Lima, L.C.P. 642 (HUEFS) \\
\hline \multicolumn{4}{|l|}{ Phytolaccaceae } \\
\hline $\begin{array}{l}\text { Petiveria alliacea } \mathrm{L} . * \# \\
\text { Phytolacca rivinoides Kunth \& Bouché }\end{array}$ & ARB & $\begin{array}{l}\text { AM, CAA, MA, } \\
\text { PAM } \\
\text { AM, CAA, MA, } \\
\text { PAT }\end{array}$ & $\begin{array}{l}\text { Mendes, R.F. et al. } 32 \text { (EAFM) } \\
\text { Almeida, C. \& Castro, A. } 159 \text { (INPA) }\end{array}$ \\
\hline \multicolumn{4}{|l|}{ Picramniaceae } \\
\hline Picramnia sellowii G.Planch.* & LIA & AM, CAA, CE, MA & Almeida, T.E. 3639 (INPA) \\
\hline \multicolumn{4}{|l|}{ Piperaceae } \\
\hline Piper aduncum $\mathrm{L}$. & ARV & $\begin{array}{c}\text { AM, CAA, CE, MA, } \\
\text { PAM, PAT }\end{array}$ & Junqueira, A.B. 210 (INPA) \\
\hline Piper cyrtopodon (Miq.) C.DC. & ARB & $\mathrm{AM}$ & Almeida, C. et al. 18 (INPA) \\
\hline Piper erectipilum Yunck. & ARB & $\mathrm{AM}$ & Almeida, T.E. 3613 (INPA) \\
\hline Piper hispidum S.w & ARB & $\mathrm{AM}, \mathrm{CE}, \mathrm{MA}$ & Almeida, C. \& Castro, A. 72 (INPA) \\
\hline Piper macapaense Yunck.* & ARB & $\mathrm{AM}$ & Mota, C.D.A. da 61674 (INPA) \\
\hline Piper mollicomum Kunth & ARB & AM, CAA, CE, MA & Almeida, C. \& Castro, A. 74 (INPA) \\
\hline Piper peltatum $\mathrm{L}$. & ARB & $\mathrm{AM}, \mathrm{CE}$ & Almeida, C. et al. 84 (INPA) \\
\hline \multicolumn{4}{|l|}{ Poaceae } \\
\hline Andropogon leucostachyus Kunth & ERV & $\begin{array}{c}\text { AM, CAA, CE, MA, } \\
\text { PAM, PAT }\end{array}$ & Almeida, C. et al. 31 (INPA) \\
\hline Anthaenantia lanata (Kunth) Benth. & ERV & AM, CAA, CE, MA, & Almeida, TE et al. 3314 (BHCB) \\
\hline Cenchrus purpureus (Schumach.) Morrone\# & ERV & AM, CAA, CE, MA & Almeida, C. et al. 30 (INPA) \\
\hline Coleataenia stenodes (Griseb.) Soreng & ERV & $\mathrm{AM}, \mathrm{CE}$ & Carvalho, F.A. de 2008 (INPA) \\
\hline Cymbopogon citratus (DC.) Stapf*\# & ERV & $\begin{array}{l}\text { AM, CAA, CE, MA } \\
\text { AM, CAA, CE, MA, }\end{array}$ & Mendes, R.F. et al. 11 (EAFM) \\
\hline Eragrostis hypnoides (Lam.) Britton, Sterns \& Poggenb. & ERV & PAM & Silva, G.P. 339 (CEN) \\
\hline Homolepis cf aturensis (Kunth) Chase & ERV & $\begin{array}{c}\text { AM, CAA, CE } \\
\text { AM, CAA, CE, MA, }\end{array}$ & Almeida, C. \& Castro, A. 213 (INPA) \\
\hline Ichnanthus calvescens (Nees ex Trin.) Döll & ERV & PAT & Almeida, C. \& Castro, A. 25 (INPA) \\
\hline Olyra latifolia $\mathrm{L}$. & ERV & AM, CAA, CE, MA & Almeida, C. \& Castro, A. 132 (INPA) \\
\hline Panicum capillare $\mathrm{L} . * * \#$ & ERV & PAM & Almeida, C. et al. 46 (INPA) \\
\hline Pariana ulei Pilg. & ERV & $\mathrm{AM}$ & Ferreira, C.A.C. 5616 (INPA) \\
\hline Paspalum gardnerianum Nees & ERV & $\begin{array}{c}\text { AM, CAA, CE } \\
\text { AM, CAA, CE, MA, }\end{array}$ & Almeida, C. \& Castro, A. 105 (INPA) \\
\hline Urochloa fusca (Sw.) B.F.Hansen \& Wunderlin\# & ERV & PAT & Almeida, C. \& Castro, A. 39 (INPA) \\
\hline \multicolumn{4}{|l|}{ Polygalaceae } \\
\hline Bredemeyera myrtifolia (A.W.Benn.) Marques & ARB & $\mathrm{AM}$ & Ferreira, C.A.C. 5583 (INPA) \\
\hline Caamembeca spectabilis (DC.) J.F.B.Pastore & $\mathrm{ARB}$ & AM, CAA, MA & Almeida, C.\& Castro, A. 127 (INPA) \\
\hline Polygala adenophora DC. & SUB & AM, CE, MA & Ferreira, C.A.C. 5524 (INPA) \\
\hline
\end{tabular}




\begin{tabular}{|c|c|c|c|}
\hline Scientific name & Habit & $\begin{array}{l}\text { Phytogeographic } \\
\text { domain }\end{array}$ & Voucher \\
\hline \multicolumn{4}{|l|}{ Pontederiaceae } \\
\hline Eichhornia crassipes (Mart.) Solms & ERV & $\begin{array}{c}\text { AM, CAA, CE, MA, } \\
\text { PAM, PAT }\end{array}$ & Almeida, C. \& Castro, A. 189 (INPA) \\
\hline \multicolumn{4}{|l|}{ Proteaceae } \\
\hline Panopsis rubescens (Pohl) Rusby & ARB & $\mathrm{AM}, \mathrm{CE}, \mathrm{MA}$ & Ferreira, C.A.C. 5540 (INPA) \\
\hline \multicolumn{4}{|l|}{ Rapateaceae } \\
\hline Cephalostemon gracilis (Poepp. \& Endl.) R.H.Schomb. & ERV & AM & Ferreira, C.A.C. 5794 (INPA) \\
\hline Rapatea paludosa Aubl. & ERV & AM, MA & Ferreira, C.A.C. 5587 (INPA) \\
\hline Rapatea spectabilis Pilg. & ERV & AM & Mota, C.D.A. da 61675 (INPA) \\
\hline \multicolumn{4}{|l|}{ Rhamnaceae } \\
\hline Ampelozizyphus amazonicus Ducke. & LIA & $\mathrm{AM}$ & Mendes, R.F. et al. 25 (EAFM) \\
\hline \multicolumn{4}{|l|}{ Rubiaceae } \\
\hline Alibertia edulis (Rich.) A.Rich. & $\mathrm{ARB}$ & $\mathrm{AM}, \mathrm{CE}$ & Silveira, A.L.P. 266 (UEC) \\
\hline Alibertia edulis (Rich.) A.Rich. var. edulis & ARB & $\mathrm{AM}, \mathrm{CE}$ & Ferreira, C.A.C. 5605 (INPA) \\
\hline Coffea liberica Hiern\# & $\mathrm{ARB}$ & AM, MA & Almeida, C. et al. 79 (INPA) \\
\hline Cordiera myrciifolia (K.Schum.) C.H.Perss. \& Delprete. & ARB & AM, CAA, CE, MA & Silveira, A.L.P. 261 (RON) \\
\hline Faramea capillipes Múll.Arg. & $\mathrm{ARB}$ & $\mathrm{AM}$ & Ferreira, C.A.C. 5770 (INPA) \\
\hline Faramea occidentalis (L.) A.Rich. & $\mathrm{ARB}$ & $\mathrm{AM}$ & Ferreira, C.A.C. 5607 (INPA) \\
\hline Ferdinandusa rudgeoides (Benth.) Wedd. & ARV & $\mathrm{AM}, \mathrm{CE}$ & $\begin{array}{l}\text { Junqueira, A.B. \& Neto, R.F. } 543 \\
\text { (EAFM) }\end{array}$ \\
\hline Isertia hypoleuca Benth. & ARV & $\mathrm{AM}$ & Almeida, C. \& Castro, A. 23 (INPA) \\
\hline Kutchubaea insignis Fisch. ex DC. & $\mathrm{ARB}$ & AM & Ferreira, C.A.C. 5609 (INPA) \\
\hline Mussaenda erythrophylla Schumach \& Thonn. \#\# & $\mathrm{ARB}$ & $\mathrm{AM}, \mathrm{CE}, \mathrm{MA}$ & Almeida, C. \& Castro, A. 188 (INPA) \\
\hline Pagamea coriacea Spruce ex Benth. & ARV & AM & Vicentini, A. 2211 (INPA) \\
\hline Palicourea amapaensis Steyerm. & ARV & $\mathrm{AM}$ & Ferreira, C.A.C. 5760 (INPA) \\
\hline Palicourea bracteosa Standl. & ARB & AM & Mota, C.D.A. da 61550 (INPA) \\
\hline Palicourea charianthema Standl. & ARB & AM & Almeida, C. \& Castro, A. 163 (INPA) \\
\hline Palicourea corymbifera (Müll.Arg.) Standl. & $\mathrm{ARB}$ & $\mathrm{AM}$ & Almeida, C. \& Castro, A. 166 (INPA) \\
\hline $\begin{array}{l}\text { Palicourea fastigiata Kunth } \\
\text { Palicourea grandifolia (Willd. ex Roem. \& Schult.) }\end{array}$ & $\mathrm{ARB}$ & AM & Viana, G.P. 62 (INPA) \\
\hline Standl. & ARV & AM & Junqueira, A.B. 224 (INPA) \\
\hline Palicourea guianensis Aubl. & ARV & AM, CAA, CE, MA & Junqueira, A.B. 305 (INPA) \\
\hline Palicourea lasiantha K.Krause & $\mathrm{ARB}$ & $\mathrm{AM}$ & Almeida, C. \& Castro, A. 168 (INPA) \\
\hline Palicourea marcgravii A.St.-Hil* & $\mathrm{ARB}$ & AM, CAA, CE, MA & Almeida, C. \& Castro, A. 139 (INPA) \\
\hline Palicourea racemosa (Aubl.) Borhidi & ARB & $\mathrm{AM}, \mathrm{CE}, \mathrm{MA}$ & Almeida, C. \& Castro, A. 164 (INPA) \\
\hline Perama hirsuta Aubl. & SUB & AM, CAA, CE, MA & Ferreira, C.A.C. 5528 (INPA) \\
\hline Psychotria apoda Steyerm. & SUB & $\mathrm{AM}$ & $\begin{array}{l}\text { Almeida, C. \& Castro, A. } 70 \text { (INPA) } \\
\text { Almeida, C \& Turma CB15 ME01 } 187\end{array}$ \\
\hline Psychotria bracteocardia (DC.) Müll.Arg. & $\mathrm{ARB}$ & AM, CAA, CE, MA & (INPA) \\
\hline Psychotria iodotricha Müll.Arg. & ARB & AM, CAA, MA & Almeida, C. et al. 19 (INPA) \\
\hline Psychotria platypoda DC. & $\mathrm{ARB}$ & AM, CAA, CE, MA & Mota, C.D.A. da 61574 (INPA) \\
\hline Psychotria rosea (Benth.) Múll.Arg. & $\mathrm{ARB}$ & AM & Ferreira, C.A.C. 5840 (INPA) \\
\hline Psychotria subundulata Benth. & $\mathrm{ARB}$ & $\mathrm{AM}, \mathrm{CE}$ & Viana, G.P. 66 (INPA) \\
\hline Psyllocarpus campinorum (Krause) J.H. Kirkbr. & $\mathrm{ARB}$ & AM & Ferreira, C.A.C. 5815 (MO) \\
\hline Rudgea lanceifolia Salisb. & ARB & $\mathrm{AM}$ & Ferreira, C.A.C. 5615 (INPA) \\
\hline Sabicea cf velutina Benth & TRE & AM & Almeida, C. \& Castro, A. 156 (INPA) \\
\hline Tocoyena guianensis K.Schum. & ARB & AM & Almeida, C. \& Castro, A. 243 (INPA) \\
\hline Uncaria guianensis (Aubl.) J.F.Gmel.* & $\mathrm{ARB}$ & $\mathrm{AM}, \mathrm{CE}$ & Ferreira, C.A.C. 5825 (INPA) \\
\hline Warszewiczia coccinea (Vahl) Klotzsch & $\mathrm{ARB}$ & $\mathrm{AM}, \mathrm{CAA}$ & Almeida, C. \& Castro, A. 136 (INPA) \\
\hline \multicolumn{4}{|l|}{ Rutaceae } \\
\hline Citrus $x$ aurantium L. **\# & ARB & CE, MA & Mendes, R.F. et al. 51 (EAFM) \\
\hline Conchocarpus grandis Kallunki & ARB & $\mathrm{AM}$ & Mota, C.D.A. da 61661 (INPA) \\
\hline Raputia ulei (K.Krause) Kallunki & ARV & $\mathrm{AM}$ & Ferreira, C.A.C. 5767 (INPA) \\
\hline
\end{tabular}


Table 1: continuation.

\begin{tabular}{|c|c|c|c|}
\hline Scientific name & Habit & $\begin{array}{c}\text { Phytogeographic } \\
\text { domain }\end{array}$ & Voucher \\
\hline \multicolumn{4}{|l|}{ Santalaceae } \\
\hline Phoradendron poeppigii (Tiegh.) Kuijt & ERV & $\mathrm{AM}$ & Krukoff, B.A. 6011 (NY) \\
\hline \multicolumn{4}{|l|}{ Salicaceae } \\
\hline Casearia arborea (Rich.) Urb. & $\mathrm{ARB}$ & AM, CE, MA & $\begin{array}{l}\text { Almeida, C. \& Castro, A. } 225 \text { (INPA) } \\
\text { Junqueira, A.B. \& Barros, R.N.S. } 383 \\
\text { (EAFM) }\end{array}$ \\
\hline Casearia grandiflora Cambess. & ARV & AM, CAA, CE, MA & Junqueira, A.B. 627 (INPA) \\
\hline Casearia javitensis Kunth & ARV & AM, CAA, CE, MA & Junqueira, A.B. 638 (INPA) \\
\hline Casearia pitumba Sleumer & ARB & $\mathrm{AM}, \mathrm{CE}$ & Albuquerque, B.W.P. de 367 (INPA) \\
\hline Casearia ulmifolia Vahl ex Vent. & ARV & $\mathrm{AM}, \mathrm{CAA}, \mathrm{CE}$ & $\begin{array}{l}\text { Junqueira, A.B. } 655 \text { (INPA) } \\
\text { Junqueira, A.B. \& Neto, R.F. } 411\end{array}$ \\
\hline Laetia procera (Poepp.) Eichler & ARV & $\mathrm{AM}, \mathrm{CE}$ & $(\mathrm{EAFM})$ \\
\hline Hasseltia floribunda Kunth & $\mathrm{ARB}$ & AM & Viana, G.P. 75 (INPA) \\
\hline \multicolumn{4}{|l|}{ Sapindaceae } \\
\hline Allophylus pilosus (J.F.Macbr.) A.H.Gentry & ARB & AM & Albernaz, A.L.K.M. 174 (INPA) \\
\hline Allophylus punctatus (Poepp.) Radlk. & $\mathrm{ARB}$ & $\mathrm{AM}, \mathrm{CE}$ & $\begin{array}{l}\text { Ferreira, C.A.C. } 5611 \text { (INPA) } \\
\text { Junqueira, A.B. \& Barros, R.N.S. } 334\end{array}$ \\
\hline Cupania rubiginosa (Poir.) Radlk. & ARV & $\mathrm{AM}, \mathrm{CE}$ & $\begin{array}{l}\text { (EAFM) } \\
\text { Junqueira, A.B. \& Souza, S.A. } 683\end{array}$ \\
\hline Cupania scrobiculata Hook. \& Arn. & ARV & AM, MA & (EAFM) \\
\hline Matayba purgans Radlk. & ARV & $\mathrm{AM}, \mathrm{CE}$ & Krukoff, B. A. 6059 (US) \\
\hline Paullinia caloptera Radlk. & TRE & $\mathrm{AM}$ & Ferreira, C.A.C. 5775 (INPA) \\
\hline Paullinia dasygonia Radlk. & TRE & AM & Almeida, C. \& Castro, A. 113 (INPA) \\
\hline Paullinia dasystachya Radlk. & LIA & $\begin{array}{c}\text { AM } \\
\text { AM, CAA, CE, MA, }\end{array}$ & Almeida, C. \& Castro, A. 217 (INPA) \\
\hline Paullinia elegans Cambess. & ARB & PAT & $\begin{array}{l}\text { Almeida, C. \& Castro, A. } 160 \text { (INPA) } \\
\text { Junqueira, A.B. \& Barros, R.N.S. } 336\end{array}$ \\
\hline Pseudima frutescens Radlk. & ARV & AM, MA & $(\mathrm{EAFM})$ \\
\hline Talisia cerasina (Benth.) Radlk. & ARV & AM, CE, MA & $\begin{array}{l}\text { Krukoff, B. A. } 6000 \text { (NY) } \\
\text { Junqueira, A.B. \& Barros, R.N.S. } 343\end{array}$ \\
\hline Talisia guianensis Aubl. & ARV & $\mathrm{AM}$ & $(\mathrm{EAFM})$ \\
\hline \multicolumn{4}{|l|}{ Sapotaceae } \\
\hline Ecclinusa lanceolata (Mart. \& Eichler) Pierre. & ARV & AM & $\begin{array}{l}\text { Junqueira, A.B. \& Souza, S.A. } 651 \\
\text { (EAFM) }\end{array}$ \\
\hline Elaeoluma schomburgkiana (Miq.) Baill. & $\mathrm{ARB}$ & $\mathrm{AM}$ & Mota, C.D.A. da 61556 (INPA) \\
\hline Manilkara elata (Allemão ex Miq.) Monach. & ARV & AM, MA & Amoêdo, S.C. et al. 42 (EAFM) \\
\hline Manilkara inundata (Ducke) Ducke & ARV & $\mathrm{AM}$ & Viana, G.P. 82 (INPA) \\
\hline Micropholis egensis (A.DC.) Pierre & ARV & $\mathrm{AM}$ & Viana, G.P. 81 (INPA) \\
\hline Micropholis guyanensis (A.DC.) Pierre & ARV & AM, CE, MA & Mota, C.D.A. da 61459 (INPA) \\
\hline Micropholis guyanensis (A.DC.) Pierre subsp. Guyanensis & ARV & $\mathrm{AM}, \mathrm{CE}, \mathrm{MA}$ & Mota, C.D.A. da 61660 (INPA) \\
\hline Micropholis melinoniana Pierre & ARV & $\mathrm{AM}$ & Viana, G.P. 61 (INPA) \\
\hline Micropholis venulosa (Mart. \& Eichler) Pierre & $\mathrm{ARB}$ & AM, CE, MA & $\begin{array}{l}\text { Viana, G.P. } 72 \text { (INPA) } \\
\text { Junqueira, A.B. \& Neto, R.F. } 562\end{array}$ \\
\hline Pouteria caimito (Ruiz \& Pav.) Radlk. & ARV & AM, CE MA & $(\mathrm{EAFM})$ \\
\hline Pouteria elegans (A.DC.) Baehni & ARV & $\mathrm{AM}$ & Viana, G.P. 404 (INPA) \\
\hline Pouteria glomerata (Miq.) Radlk. & ARV & AM, CE, MA & $\begin{array}{l}\text { Ferreira, C.A.C. } 5769 \text { (INPA) } \\
\text { Junqueira, A.B. \& Neto, R.F. } 540\end{array}$ \\
\hline Pouteria macrophylla (Lam.) Eyma. & ARV & $\mathrm{AM}, \mathrm{CE}, \mathrm{MA}$ & $(\mathrm{EAFM})$ \\
\hline Pouteria opposita (Ducke) T.D.Penn. & ARV & $\mathrm{AM}$ & Mota, C.D.A. da 61605 (INPA) \\
\hline Sarcaulus brasiliensis (A.DC.) Eyma subsp. Brasiliensis & ARV & AM, MA & Krukoff, B. A. 6009 (NY) \\
\hline \multicolumn{4}{|l|}{ Scrophulariaceae } \\
\hline Lindernia crustacea (L.) F.Muell. & TRE & $\begin{array}{l}\text { AM, CAA, CE, MA, } \\
\text { PAM, PAT }\end{array}$ & Ferreira, C.A.C. 5792 (INPA) \\
\hline \multicolumn{4}{|l|}{ Simaroubaceae } \\
\hline Homalolepis cedron (Planch.) Devecchi \& Pirani & ARV & AM & $\begin{array}{l}\text { Junqueira, A.B. \& Neto, R.F. } 579 \\
\text { (EAFM) }\end{array}$ \\
\hline Simaba polyphylla (Cavalcante) W.W.Thomas & ARV & AM, MA & Junqueira, A.B. \& Souza, S.A. 633 \\
\hline
\end{tabular}


(EAFM)

Table 1: continuation.

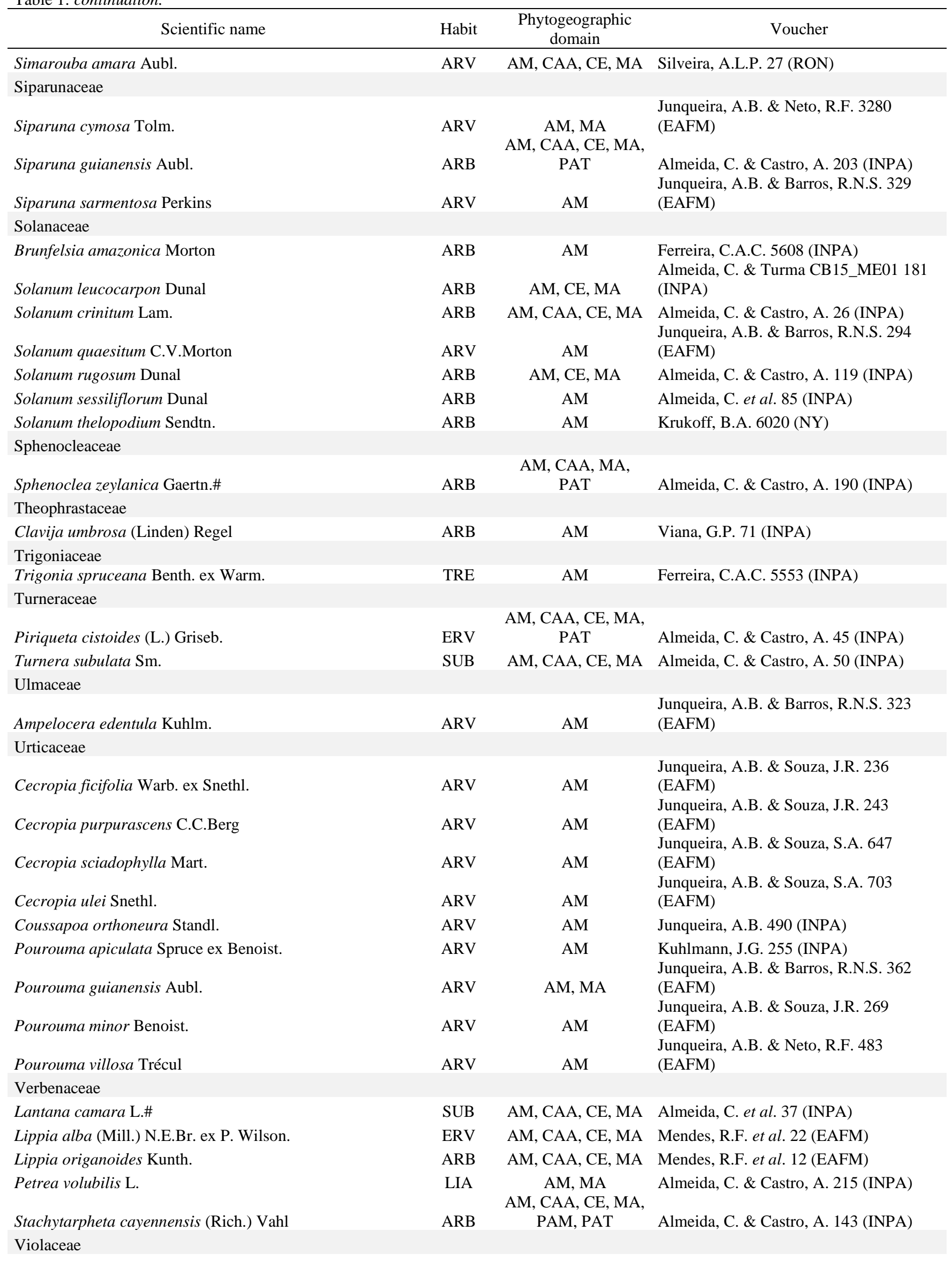


Table 1: continuation.

\begin{tabular}{|c|c|c|c|}
\hline Scientific name & Habit & $\begin{array}{c}\text { Phytogeographic } \\
\text { domain }\end{array}$ & Voucher \\
\hline Leonia crassa L.B.Sm. \& A.Fernández & ARV & AM & $\begin{array}{l}\text { Junqueira, A.B. \& Souza, J.R. } 246 \\
\text { (EAFM) }\end{array}$ \\
\hline Leonia cymosa Mart. & ARV & $\mathrm{AM}$ & Ferreira, C.A.C. 5610 (INPA) \\
\hline Paypayrola grandiflora Tul. & ARV & AM, MA & $\begin{array}{l}\text { Junqueira, A.B. \& Souza, S.A. } 665 \\
\text { (EAFM) }\end{array}$ \\
\hline Rinorea neglecta Sandwith & ARB & $\mathrm{AM}$ & Mota, C.D.A. da 61577 (INPA) \\
\hline Rinoreocarpus ulei (Melch.) Ducke & $\mathrm{ARB}$ & $\mathrm{AM}$ & Viana, G.P. 70 (INPA) \\
\hline \multicolumn{4}{|l|}{ Vitaceae } \\
\hline Cissus erosa Rich. & TRE & $\mathrm{AM}, \mathrm{CAA}, \mathrm{CE}, \mathrm{MA}$ & Almeida, C. et al. 29 (INPA) \\
\hline Cissus paraensis Lombardi & LIA & $\mathrm{AM}$ & Ferreira, C.A.C. 5531 (INPA) \\
\hline \multicolumn{4}{|l|}{ Vochysiaceae } \\
\hline Qualea dinizii Ducke* & ARV & $\mathrm{AM}$ & Amoêdo, S.C. et al. 40 (EAFM) \\
\hline Qualea grandiflora Mart. & ARV & $\mathrm{AM}, \mathrm{CAA}, \mathrm{CE}, \mathrm{MA}$ & Almeida, T.E. 3322 (BHCB) \\
\hline Qualea parviflora Mart. & ARB & AM, CAA, CE, MA & Silveira, A.L.P. 227 (UEC) \\
\hline Ruizterania retusa (Spruce ex Warm.) Marc.-Berti & ARB & $\mathrm{AM}$ & Ferreira, C.A.C. 5517 (INPA) \\
\hline Salvertia convallariodora A.St.-Hil. & ARV & $\mathrm{AM}, \mathrm{CAA}, \mathrm{CE}, \mathrm{MA}$ & Silveira, A.L.P. 44 (RON) \\
\hline Vochysia haenkeana Mart. & $\mathrm{ARB}$ & $\mathrm{AM}, \mathrm{CE}$ & Ferreira, C.A.C. 5513 (INPA) \\
\hline Vochysia maxima Ducke.* & ARV & AM & $\begin{array}{l}\text { Junqueira, A.B. \& Neto, R.F. } 466 \\
\text { (EAFM) }\end{array}$ \\
\hline Vochysia obscura Warm. & ARV & $\mathrm{AM}$ & Ferreira, C.A.C. 5561 (INPA) \\
\hline \multicolumn{4}{|l|}{ Xyridaceae } \\
\hline Abolboda grandis Griseb. & ERV & AM & Carvalho, F.A. de 1986 (INPA) \\
\hline Abolboda macrostachya Spruce ex Malme & ARB & $\mathrm{AM}$ & Mota, C.D.A. da 61564 (INPA) \\
\hline Xyris ferreirae Kral & ERV & AM & Ferreira, C.A.C. 5506 (INPA) \\
\hline Xyris lanulobractea Steyerm. & ARB & $\mathrm{AM}$ & Mota, C.D.A. da 61561 (INPA) \\
\hline Xyris pallidula Kral \& Wand. & ARB & AM & Mota, C.D.A. da 61565 (INPA) \\
\hline Xyris stenocephala Malme & ERV & AM, CE, MA & Ferreira, C.A.C. 5788 (INPA) \\
\hline Xyris uleana Malme & ERV & $\mathrm{AM}, \mathrm{CE}$ & Ferreira, C.A.C. 5809 (INPA) \\
\hline \multicolumn{4}{|l|}{ Zingiberaceae } \\
\hline Alpinia zerumbet (Pers.) B.L.Burtt \& R.M.Sm\#\# & ERV & $\mathrm{AM}, \mathrm{CAA}, \mathrm{CE}, \mathrm{MA}$ & Almeida, C. et al. 80 (INPA) \\
\hline Renealmia breviscapa Poepp. \& Endl. & ERV & AM & Ferreira, C.A.C. 5618 (INPA) \\
\hline Zingiber officinale Roscoe\#\# & ERV & DESC & Mendes, R.F. et al. 4 (EAFM) \\
\hline
\end{tabular}

\section{Conclusion}

This study highlights the importance of floristic studies in the Brazilian Amazon. Given the well-known sample deficiency of the Amazon region, and considering the countless anthropogenic pressures that cities south of the Madeira river have been facing, this preliminary angiosperm checklist provides the first tool for further botanical studies in this region. The survey highlights the record of 47 new occurrences for Amazonas State. Of these new occurrences, 12 are also the first record for the northern region. In addition, a new species of vine of the genus Mandevilla Lindl was identified.

\section{Acknowledgements}

The first author thanks the University of Amazonas State for providing opportunity for the development of this project. To Amauri Castro, Arnoldo Azevedo, Ellen Passos, Lucy Laura, Jeimiciane Coutinho, Jone Bitencourt, Neiza Paixão, Nilson Pinheiro, and Raimunda Albertina for the company and support during botanical expeditions. The authors thank Cassio Toledo for identifying Connaraceae species, and anonymous experts for their contribution in identifying some species. To Michael Hopkins and Mariana Mosque for access to the INPA Herbarium collection and for their attention and support. To Jefferson R. Maciel for preparing the distribution map of angiosperms in Manicoré city, and to Fernanda Cabral for reviewing the abstract. The last author thanks CAPES for the postdoctoral scholarship granted.

\section{References}

Amaral, I. L. do.; Matos, F. D. A. \& Lima, J. (2000). Composição florística e parâmetros estruturais de um hectare de floresta densa de terra firme no rio Uatumã, Amazônia, Brasil. Acta Amazônica, 30(3), 377-377. doi: 10.1590/1809-43922000303392.

Antonelli, A.; Zizka, A.; Carvalho, F. A.; Scharn, R.; Bacon, C.D.; Silvestro, D. \& Condamine, F. L. (2018). Amazonia is the primary source of Neotropical biodiversity. Proceedings of the National Academy of Sciences of the United States of America, 115(23), 6034-6039. doi: 10.1073/pnas.1713819115.

Araújo, R. A. (2011). Florística e estrutura da comunidade arbórea em fragmento florestal urbano no município de Sinop, Mato Grosso. (Dissertação de Mestrado). Universidade Federal de Mato Grosso, Cuiabá, Mato Grosso. Recuperado de http://www.dominiopublico.gov.br/pesquisa/DetalheObraForm.do?sel ect_action $=\&$ co_obra $=127174$.

Baitello, J. B.; Arzolla, F.A. R. dal P. \& Vilela, F. E. S. P. (2017). Nova 
espécie de Lauraceae da Floresta Ombrófila Densa Alto Montana, Serra da Mantiqueira, Pindamonhangaba, SP, Brasil. Rodriguésia, 68(2) 481488. doi: 10.1590/2175-7860201768212.

Batalha-Filho, H. \& Miyaki, C. Y. (2014). Processos evolutivos na Amazônia e na Mata Atlântica. FRONTEIRAS: Journal of Social, Technological and Environmental Science, 3(2), 34-44. doi: 10.21664/22388869.2014v3i2.p34-44.

BFG (the Brazil Flora Group) (2015). Growing knowledge: an overview of Seed Plant diversity in Brazil. Rodriguésia, 66(4), 1085-1113. doi: 10.1590/2175-7860201566411.

Brasil (1978). Ministério das Minas e Energia Departamento Nacional da Produção Mineral: Projeto Radam Brasil - folha SB. 20 - Purus (Levantamento de recursos naturais, v. 17). Rio de Janeiro: DNPM. Recuperado https://www.bdpa.cnptia.embrapa.br/consulta/busca?b=ad\&id=295969\& biblioteca=vazio\&busca=autoria:\%22PROJETO\%20RADAMBRASIL. $\% 22 \& q F a c e t s=$ autoria:\%22PROJETO\%20RADAMBRASIL.\%22\&sort $=\&$ paginacao $=\mathrm{t} \&$ paginaAtual $=3$.

Campos, M. C. C.; Ribeiro, M. R.; Souza-Júnior, V. S.; Ribeiro-Filho, M. R. \& Almeida, M. C. (2012). Relações Solo-Superfície Geomórfica em uma Topossequência Várzea-Terra Firme na Região de Humaitá (AM). Revista Brasileira de Ciência do Solo, 36, 325-336. doi: 10.1590/S010006832012000200002 .

Carvalho, J.B. de (2010). Desmatamentos, grilagens e conflitos agrários no Amazonas ( $1^{\mathrm{a}}$ ed.). Manaus: Editora Valer.

Chaves, A. C. G.; Santos, R. M. S.; Santos, J. O.; Fernandes, A. A. \& Maracajá, P. B. (2013). A importância dos levantamentos florístico e fitossociológico para a conservação e preservação das florestas. Agropecuária Científica no Semiárido (ACSA), 9(2), 43-48. doi: 10.30969/acsa.v9i2.449.

Coelho, C. A. \& Amorim, B. S. (2019). Expandindo a distribuição geográfica de Hibiscus sabdariffa L. (Malvaceae): uma espécie naturalizada negligenciada para a flora brasileira. Hoehnea, 46(1), 1-7. doi: 10.1590/2236-8906-101/2018.

Coelho, C. A.; Amorim, B. S.; Pereira, M. R. S.; Cabral, F.N.; Albuquerque, P.M. \& Morales, J. F. (in press) An overview of the Mandevilla rugellosa complex (Apocynaceae) in the Brazilian Amazonia, with a new species.

Centro de Referência e Informação Ambiental - CRIA (2018). Specieslink simple search. 2018. Recuperado de http://inct.splink.org.br/.

Domingos, C.; Särkinen, T.; Alexander, S.; Amorim, A. M.; Bittrich, V.; Celis, M.; Daly, D. C.; Fiaschi, P.; Funk, V. A.; Giacomin, L. L.; Goldenberg, R.; Heiden, G.; Iganci, J.; Kelloff, C. L.; Knapp, S. K.; Lima, H. C.; Machado, A. F. P.; Santos, R. M.; Mello-Silva, R.; Michelangeli, F. A.; Mitchel, J.; Luar, P. L.; Moraes, P. L. R.; Mori, S. A.; Nunes, T. S.; Pennington, T. D.; Pirani, J. R.; Prance, G.T.; Queiroz, L. P.; Rapini, A.; Riina, R.; Rincon, C. A. V.; Roque, N.; Shimizu, G.; Sobral, M.; Stehmann, J. R.; Stevens, W.D.; Taylor, C.M.; Trovó, M.; Berg, C. van der.; Werff, H. van der.; Viana, P.L.; Zartman, C.E. \& Forzza, R. C. (2017). Amazon plant diversity revealed by a taxonomically verified species list. Proceedings of the National Academy of Sciences of the United States of America, 114(40), 10695-10700. doi: 10.1073/pnas.1706756114.

Esteves, G. L.; Duarte, M. C. \& Takeuchi, C. (2014). Sinopse de Hibiscus L. (Malvoideae, Malvaceae) do Estado de São Paulo, Brasil: espécies nativas e cultivadas ornamentais. Hoehnea, 41(4), 529-539. doi: 10.1590/22368906-10/2014.

Fabaceae in Flora do Brasil 2020 em construção (2018). Jardim Botânico do Rio de Janeiro. Recuperado de http://floradobrasil.jbrj.gov.br/.

Fearnside, P. M. (2006). Desmatamento na Amazônia: dinâmica, impactos e controle. Acta Amazônica, 36(3), 395-400. doi: 10.1590/S004459672006000300018.

Ferreira, L. V.; Chaves, P. P.; Cunha, D. de A.; Rosário, A. S. do \& Parolin, P. (2013). A extração ilegal de areia como causa do desaparecimento de campinas e campinaranas no estado do Pará, Brasil. Pesquisas Botânicas, 64, 157-173. Recuperado de http://www.anchietano.unisinos.br/publicacoes/botanica/botanica64/10_f erreira\%20e\%20parolin.pdf.

Fiaschi, P. \& Pirani, J. R. (2009). Review of plant biogeographic studies in Brazil. Journal of Systematics and Evolution, 47(5), 477-496. doi: 10.1111/j.1759-6831.2009.00046.x.

Flora do Brasil 2020 em construção (2018). Jardim Botânico do Rio de Janeiro. Recuperado de http://floradobrasil.jbrj.gov.br/.

Forzza, R. C.; Baumgratz, J. F.; Costa, A.; Hopkins, M.; Leitman, P.M.; Lohmann, L. G.; Martinelli, G.; Morim, M. P.; Coelho, M. A. N.; Peixoto, A. I.; Pirani, J. R.; Queiroz, L. P.; Stehmann, J. R.; Walter, B. M. T. \& Zappi, D. (2010). Introdução as Angiospermas do Brasil. Catálogo de plantas e fungos do Brasil [online]. Rio de Janeiro. Recuperado de http://reflora.jbrj.gov.br/downloads/vol1.pdf.

Garcia, L. M.; Silva, R. F.; Zonetti, P. C. \& Romagnolo, M. B. (2011). Levantamento florístico e fitossociológico de um remanescente de mata ciliar na região norte do estado do Paraná, Brasil._Anais eletrônicos. VII EPCC Encontro Internacional de Produção Científica Cesumar. Recuperado http://www.cesumar.br/prppge/pesquisa/epcc2011/anais/leticia_monic a_garcia.pdf.

Gonçalves, E. G. \& Lorenzi, H. Morfologia Vegetal: Organografia e Dicionário Ilustrado de Morfologia das Plantas Vasculares ( $1^{\mathrm{a}}$ ed. $)$. São Paulo: Instituto Plantarum de Estudos da Flora.

Gonçalves, F. G. \& Santos, J. R. (2008). Composição florística e estrutura de uma unidade de manejo florestal sustentável na Floresta Nacional do Tapajós, Pará. Acta Amazônica, 38(2), 229-244. doi: 10.1590/S004459672008000200006

Google Earth. (2019). V 7.3.2.5776 (64-bit). Manicoré, Amazonas, Brasil. 28 maio 2019. Image Landsat/Copernicus 2019.

Hibiscus in Flora do Brasil 2020 em construção. (2019). Jardim Botânico do Rio de Janeiro. Recuperado de http://floradobrasil.jbrj.gov.br/reflora/floradobrasil/FB9079.

Hopkins, M. J. G. (2005). Flora da Reserva Ducke, Amazonas, Brasil. Rodriguésia, 56(86), 9-25. doi: 10.1590/1809-43921994242030.

Hopkins, M. J. G. (2007). Modelling the known and unknown plant biodiversity of the Amazon Basin. Journal of Biogeography, 34(8), 1400-1411. doi: 10.1111/j.1365-2699.2007.01737.x

Hopkins, M. J. G. (2019). Are we close to knowing the plant diversity of the Amazon? Anais da Academia Brasileira de Ciências. 91(3), 1-7. doi: 10.1590/0001-3765201920190396.

IBGE - Instituto Brasileiro de Geografia e Estatística. (2004). Mapa de vegetação do Brasil. Recuperado de https://www.ibge.gov.br.

IBGE - Instituto Brasileiro de Geografia e Estatística. (2017). Mapa de localização do município de Manicoré. Recuperado de https://cidades.ibge.gov.br/brasil/am/manicore/panorama.

Ivanauskas, N. M.; Monteiro, R. \& Rodrigues, R. R. (2004). Composição florística de trechos florestais na borda sul-amazônica. Acta Amazônica, 34(3), 399-413. doi: 10.1590/S0044-59672004000300006.

Jardim Botânico do Rio de Janeiro - JABOT. (2019). Banco de Dados da Flora Brasileira: Consulta, 2018. Recuperado de http://rb.jbrj.gov.br/v2/consulta.php.

Junqueira, A.B. (2008). Uso e manejo da vegetação secundária sobre terra preta por comunidades tradicionais na região do médio rio Madeira, Amazonas, Brasil. (Dissertação de mestrado). Instituto Nacional de Pesquisas da Amazônia/ Universidade Federal do Estado do Amazonas, Manaus, Amazonas. Recuperado https://bdtd.inpa.gov.br/handle/tede/953\#preview-link0.

Kubitski, K. (1979). Ocorrência de Kielmeyera nos " campos de Humaitá" e a natureza dos " campos" - Flora da Amazônia. Acta amazônica, 9(2), 401-404. doi: 10.1590/1809-43921979092401.

Lopes, C. R. A. S.; Ribeiro, R. da S.; Rodrigues, L.; Cabral, F.F. \& Silva, D. R. (2014). Checklist de angiospermas da região de influência da Uhe Sinop, médio Teles Pires, Mato Grosso. Enciclopédia Biosfera, Centro Científico Conhecer, 10(19), 2036-2048. doi: 10.13140/RG.2.1.3774.8323.

Martins, G. C.; Ferreira, M.M.; Curi, N.; Vitorino, A. C. T. \& Silva, M. L. N. (2006). Campos nativos e matas adjacentes da região de Humaitá (AM): atributos diferenciais dos solos. Ciência e Agrotecnologia, 30(2), 221-227. doi: 10.1590/S1413-70542006000200005.

Méio, B. B.; Freitas, C. V.; Jatobá, L.; Silva, M. E. F.; Ribeiro, J. F. \& Henriques, R. P. B. (2003). Influência da flora das florestas Amazônica e Atlântica na vegetação do cerrado sensu stricto. Revista Brasileira de Botânica, 26(4), 437-444. doi: 10.1590/S0100-84042003000400002.

Moro, M. F.; Araújo, F. S.; Souza, V. C.; Oliveira-Filho, A. T.; Queiroz, L. P.; Fraga, C. N.; Rodal, M. J. N. \& Martins, F. R. (2012). Alienígenas na sala: o que fazer com espécies exóticas em trabalhos de taxonomia, florística e fitossociologia? Acta Botânica Brasílica, 26(4), 991-999. doi: 10.1590/S0102-33062012000400029.

Mota, N. F. O.; Watanabe, M. T. C.; Zappi, D. C.; Hiura, A. L.; Pallos, J.; Viveros, R. S.; Giuletti, A. M. \& Viana, P. L. (2018). Cangas da Amazônia: a vegetação única de Carajás evidenciada pela lista de fanerógamas. Rodriguésia, 69(3), 1435-1488. doi: 10.1590/21757860201869336.

Oliveira, A. N.; Amaral, I. L.; Ramos, M. B. P.; Nobre, A.D.; Couto, L. B. \& Sahdo, R. M. (2008). Composição e diversidade florístico-estrutural de um hectare de floresta densa de terra firme na Amazônia Central, Amazonas, Brasil. Acta Amazônica, 38(4), 627-642. doi: 
10.1590/S0044-59672008000400005.

Oliveira, E. K. B.; Nagy, A. C. G.; Barros, Q. S.; Martins, B. C. \& MurtaJunior, L. S. (2015). Composição florística e fitossociológica de fragmento florestal no sudoeste da Amazônia. Enciclopédia Biosfera, Centro Científico Conhecer, 11(21), 2126-2146. Recuperado de http://www.conhecer.org.br/enciclop/2015b/biologicas/composicao\%20fl oristica.pdf.

Peixoto, A.L. \& Morim, M.P. (2003). Coleções botânicas: documentação da biodiversidade brasileira. Ciência e cultura, 55(3), 21-24. Recuperado de http://cienciaecultura.bvs.br/pdf/cic/v55n3/a16v55n3.pdf.

Peixoto, A. L. \& Maia, L. C. (2013). Manual de Procedimentos para Herbários. INCT-Herbário virtual para a Flora e os Fungos. Recife: Editora Universitária. Recuperado de http://inct.florabrasil.net/wpcontent/uploads/2013/11/Manual_Herbario.pdf.

Pinheiro, E. S.; Martinot, J. F.; Cavalcante, D. G. C.; Macedo, M. A.; Nascimento, A. Z. A. \& Marques, J. P. C. (2010). Paisagem, estrutura e composição florística de um parque urbano em Manaus, Amazonas, Brasil. Rodriguésia, 61(3), 531-549. doi: 10.1590/2175-7860201061313.

Pivetta, M. (2011). Ilhas de Savana. Pesquisa FAPESP, 179, 46-49. Recuperado de https://revistapesquisa.fapesp.br/wpcontent/uploads/2011/01/046-049-179.pdf.

Porto-Gonçalves, C. W. (2015). Amazônia enquanto acumulação desigual de tempos: Uma contribuição para a ecologia política da região. $\underline{\text { Revista }}$ Crítica de Ciências Sociais, 107, 63-90. doi:10.4000/rccs.6018.

Prieto, R. O. \& González-Oliva, L. (2015). Lista nacional de plantas invasoras y potencialmente invasoras en la república de Cuba. Bissea, 9(2), 1-88. Recuperado de https://www.researchgate.net/publication/284545720_LISTA_NACION AL_DE_PLANTAS_INVASORAS_Y_POTENCIALMENTE_INVASO RAS_EN_LA_REPUBLICA_DE_CUBA_-_2015.

Rapp Py-Daniel, L. (2007). Contextualização do projeto e financiamento. In: Rapp Py-Daniel, L.; Deus, C. P.; Henriques, A. L.; Pimpão, D. M.; Ribeiro, O. M. (orgs.). Biodiversidade do Médio Madeira: Bases científicas para propostas de conservação (Cap. 1, pp. 19-23). Manaus: INPA.

Ribeiro, J. E. L. S.; Nelson, B. W.; Silva, M. F.; Martins, L .S. S. \& Hopkins, M. (1994). Reserva florestal Ducke: diversidade e composição da flora vascular. Acta Amazônica, 24(1-2), 19-30. doi: 10.1590/180943921994242030.

Salles, M. C. T.; Grigio, A. M. \& Silva, M. R. F. (2013). Expansão urbana e conflito ambiental: uma descrição da problemática do município de Mossoró, RN - Brasil. Sociedade \& Natureza, 25(2), 281-290. Recuperado http://www.seer.ufu.br/index.php/sociedadenatureza/article/view/14389/ pdf.

Sardinha, M. A.; Freitas, J. L.; Santos, A. C.; Junior, F. de O. C. \& Santos, E. S. (2017). Florística e utilização de espécies florestais em assentamento agroextrativista, Amapá, Amazônia Oriental. Enciclopédia Biosfera, Centro Científico Conhecer, 14(26), 595-610. doi: 10.18677/EnciBio_2017B33.

Schneide, A. A. (2007). A flora naturalizada no estado do Rio Grande do Sul, Brasil: herbáceas subespontâneas. Biociências, 15(2), 257-268. Recuperado http://revistaseletronicas.pucrs.br/ojs/index.php/fabio/article/view/254/30 05 .

Silva, K. E. \& Pereira, K. P. (2005). Alterações na cobertura vegetal natural dos municípios do Sul do Amazonas. Anais XII Simpósio Brasileiro de Sensoriamento Remoto, 16-21. Recuperado de http://marte.sid.inpe.br/col/ltid.inpe.br/sbsr/2004/11.22.09.51/doc/1667.p df.

Silva, K. E.; Matos, F. D. A. \& Ferreira, M. M. (2008). Composição florística e fitossociologia de espécies arbóreas do Parque Fenológico da Embrapa Amazônia Ocidental. Acta Amazônica, 38(2), 213-222. doi: 10.1590/S0044-59672008000200004.

Silvério, D.; Silva, S.; Alencar, A. \& Moutinho, P. (2019). Amazônia em chamas. Nota técnica do Instituto de Pesquisa Ambiental da AmazôniaIPAM. Recuperado de: https://ipam.org.br/wpcontent/uploads/2019/08/NT-Fogo-Amazo\%CC\%82nia-2019.pdf.

Siqueira, A. G. (2013). Características e avaliação dos níveis basais de mercúrio do sedimento na área de influência do aproveitamento hidrelétrico Jirau - Rio Madeira - Rondônia - Brasil. (Dissertação de mestrado). Universidade de Brasília, Planaltina, Distrito Federal. Recuperado http://repositorio.unb.br/bitstream/10482/13908/1/2013_AlineGon\%c3\% a7alvesdeSiqueira.pdf.

Sousa, V. S.; Machado-Filho, H. O. \& Andrade, T. M. (2012). Similaridade de vegetação ruderal entre regiões do Brasil. Revista Geonorte, 3(4), 274283. Recuperado de http://www.periodicos.ufam.edu.br/revistageonorte/article/view/1825.

Ter Steege, H.; Vaessen, R. W.; López, D. C.; Sabatier, D.; Antonelli, A.; Oliveira, S. M.; Pitman, N.; Jorgensen, P. M.; Salomão, R. P. \& Gomes, V. H. F. (2016) A descoberta da flora arbórea da Amazônia com uma lista atualizada de todos os taxa arbóreos conhecidos. Boletim do Museu Paraense Emílio Goeldi, 11(2), 231-261. doi: 10.1038/srep29549.

The plant list. (2010). Version 1. Published on the Internet. Recuperado de http://www.theplantlist.org/.

Vieira, I. C. G.; Toledo, P. M. \& Higuchi, H. (2018). A Amazônia no Antropoceno. Ciência e Cultura, 70(1), 56-59. doi: 10.21800/231766602018000100015

\section{License: Creative Commons CC BY 4.0}

This article was published with open access for distribution under the terms of the Creative Commons Attribution License, which allows unrestricted use, distribution, and reproduction in any medium, provided the original work is properly cited. 